NAT'L INST OF STAND \& TECH 




National Bureau of Standards

AUG 171959

98622

TALIO

448

Cos. i 


\section{Live Loads on Floors in Buildings}

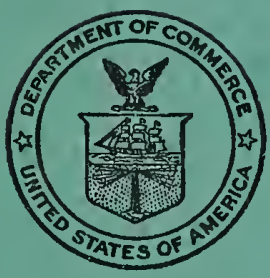

United States Department of Commerce

National Bureau of Standards

Building Materials and Structures Report 133 


\section{BUILDING MATERIALS AND STRUCTURES REPORTS}

On request, the Superintendent of Documents, U. S. Government Printing Office, Washington 25. D. C., will place your name on a special mailing list to receive notices of new reports in this series as soon as they are issued. There will be no charge for receiving such notices.

If 100 copies or more of any report are ordered at one time, a discount of 25 percent is allowed. Send all orders and remittances to the Superintendent of Documents, $U$. S. Government Printing Office, Washington 25, D. C.

The following publications in this series are available by purchase from the Superintendent of Document at the prices indicated:

BMS2 Methods of Determining the Structural Properties of Low-Cost House Constructions BMS3 BMS4

BMS5

BMS6

BMS8

Suitability of Fiber Insulating Lath as a Plaster Base

Accelerated Aging of Fiber Building Boards

Structural Properties of Six Masonry Wall Constructions

Survey of Roofing Materials in the Southeastern States

Methods of Investigation of Surface Treatment for Corrosion Protection of Steel

BMS9 Structural Properties of the Insulated Steel Construction Co.'s "Frameless-Steel" Constructions for Walls, Partitions, Floors, and Roofs

BMS10 Structural Properties of One of the "Keystone Beam Steel Floor" Constructions Sponsored by the H. H. Robertson Co.

BMS11 Structural Properties of the Curren Fabrihome Corporation's "Fabrihome" Construc-

BMS12 Structural Properties of "Steelox" Constructions for Walls, Partitions, Floors, and

BMS15 Structural Properties of "Wheeling Long-Span Steel Floor" Construction Sponsored by

BMS16 Structural Properties of a "Tilecrete" Floor Construction Sponsored by Tilecrete Floors, Inc.-

BMS17 Sound Insulation of Wall and Floor Constructions Supplement to BMS17, Sound Insulation of Wall and Floor Constructions Supplement No. 2 to BMS17, Sound Insulation of Wall and Floor Constructions
BMS18 Structural Properties of "Pre-fab" Constructions for Walls, Partitions, and Floors BMS20 Structural Properties of "Twachtman" Constructions for Walls and Floors Sponsored by Connecticut Pre-Cast Buildings Corporation

BMS21 Structural Properties of a Concrete-Block Cavity-Wall Construction Sponsored by the National Concrete Masonry Association

BMS23 Structural Properties of a Brick Cavity-Wall Construction Sponsored by the W. E. Manufacturers Association of New York, Inc..... 10

BMS24 Structural Properties of a Reinforced-Brick Wall Construction and a Brick-Tile CavityWall Construction Sponsored by the Structural Clay Products Institute._..... 15\&

BMS25 Structural Properties of Conventional Wood-Frame Constructions for Walis, Parti-

BMS26 Structural Properties of "Nelson Pre-Cast Concrete Foundation" Wall Construction Sponsored by the Nelson Cement Stone Co," Inc "Poun

BMS27 Structural Properties of "Bender Steel Home" Wall Construction Sponsored by the

BMS28 Backfow Prevention in Over-Rim Water Supplies

BMS29 Survey of Roofing Materials in the Northeastern States
BMS30 Structural Properties of a Wood-Frame Wall Construction Sponsored by the Douglas

BMS32 Structural Properties of Two Brick-Concrete-Block Wall Constructions and a ConAssociation

BMS34

Performance Test of Floor Coverings for Use in Low-Cost Housing: Part 1.

[List continued on cover page III] 


\title{
Live Loads on Floors in Buildings
}

\author{
John W. Dunham, Guttorm N. Brekke \\ and George N. Thompson
}

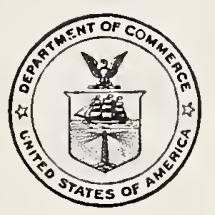

Building Materials and Structures Report 133

Issued December 19, 1952 


\section{Foreword}

This report summarizes available information on floor loads in buildings, including the results of a recent survey that has provided data on several occupancies about which detailed information has been lacking. Variations in loading within the same occupancy are shown, and a method of live-load reduction for structural members supporting large floor areas is described.

It has been found possible to increase allowable stresses for some building materials as a result of better quality control and increased knowledge of strength characteristics. However, loads and stresses are so intimately associated that good information on loads is essential in order to realize the full advantage of economical design and to conserve scarce materials. It is believed that the data presented in this report will assist in accomplishing these objectives.

\section{A. V. Astin, Director.}

\section{GONTENTS}

1. Introduction

2. Residential occupancy

3. Business occupancy Offices

Method of conducting surveys

t. Mercantile occupancy _....................

5. Assembly occupancy

Schools

Crowded rooms

6. Institutional occupanc

Hospitals

7. Industrial occupancy

Mattress factory, Chicago, Ill .............

Page
ii
1
2
3
3
4
4
8
9
9
9
10
10
11

7. Industrial occupancy-Continued Mattress factory, Atlanta, Ga _._._. ... 11

Men's clothing factory, New York, N. Y ... 13

Dress factory, Philadelphia, $\mathrm{Pa} \ldots \ldots \ldots 15$

Furniture factory, Gettysburg, $\mathrm{Pa} \ldots \ldots \ldots 15$

Furniture factory, Grand Rapids, Mich_.... 17

Newspaper plant, Washington, D. C....... 18

Printing plant, ITashington, D, C . .....

8. Storage occupancy

TIarehouse, New Fork, N. Y . . . .

Warehouse, Washington, D. C $\ldots 25$

9. Tariation in loading

10. Discussion

11. Selected references

TABLES

Page

Maximum, minimum, and average live loads in Equitable Building ..................

Office live loads in Union Central Life Insurance

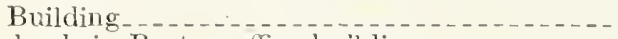

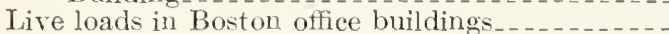

1. Live loads in department store, New York, $\mathbf{N}$. Y.

2. Tariation in live loads in department store, New

Tork, N. Y Live loads in department store, Washington, D. C.

1. Tariation in live loads in department store, Washington, D. C

Live loads in crowded wards

5. Live loads in mattress factory, Chicago, Ill .

6. Tariation in live loads in mattress factor's, Chi-

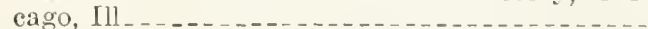

7. Live loads in mattress factory, Atlanta, Ga

8. Tariation in live loads in mattress factory,

9. Live loads in men's clothing factory, New York,

10. Tariation in live loads in men's clothing factory, New York, N. Y.
11. Live loads in dress factory, Philadelphia, $\mathrm{Pa}$

12. Variation in live loads in dress factory, Philadelphia, $\mathrm{Pa}_{\ldots} \ldots \ldots \ldots \ldots$

13. Live loads in furniture factory, Gettysburg, Pa.-

14. Tariation in live loads in furniture factory, Gettysburg, $\mathrm{Pa}_{\ldots} \ldots \ldots \ldots$

15. Live loads in furniture factory, Grand Rapids,

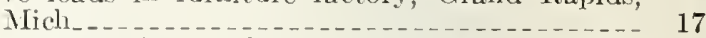

16. Variation in live loads in furniture factory, Grand Rapids, Mich _................

17. Live loads in newspaper plant, Washington, D. C-

18. Tariation in live loads in newspaper plant, Washington, D. C $\ldots \ldots$

19. Live loads in printing plant, Washington, D. C-

20. Variation in live loads in printing plant, Washington, D. C

21. Live loads in warehouse, New York, N. Y....

22. Tariation in live loads in warehouse, Tew York, T. Y

23. Live loads in warehouse, Washington, D. C...

24. Tariation in live loads in warehouse, Washington, D. C... 


\title{
Live Loads on Floors in Buildings
}

\author{
John W. Dunham,* Guttorm N. Brekke, and George N. Thompson
}

\begin{abstract}
Information is presented on floor loads found in various occupancies such as office buildings, stores, factories, and warehouses. Most of this consists of the results of a recent survey in which the weight and distribution of goods, equipment, and occupants were obtained floor by floor and area by area. Such data are needed as a check on permissible ninimum live loads in building codes and as a basis for design of buildings. Although there are indications in the survey that building codes may set higher figures than are warranted for certain occupancies, the amount of information available is still too small to justify firm conclusions. A method of reducing the assumed value for live load on structural members supporting large floor areas is described. Supplementary investigations of floor loading are recommended to clear up questions that have arisen in connection with the survey.
\end{abstract}

\section{Introduction}

Structural design of buildings is dependent upon knowledge of the loads to which such structures may be subjected and upon knowledge of building materials and structural systems. Through accumulation of data obtained in laboratory tests, much progress has been made in knowledge of the strength of building materials. The approximate weights of such materials have also been established. The characteristic behavior of different structural systems is receiving increased attention. Data on the loads affecting buildings are, howe ver, rather meager, particularly with respect to the actual loads imposed by goods, equipment, and persons in typical occupancies such as offices, stores, and factories. Building regulations are in fairly close agreement as to the minimum loads to be assumed in designing buildings that contain such occupancies, in spite of the small amount of information upon which to base such requirements. However, with increasing urgency for conservation of materials, because of considerations of both scarcity and cost, it seems advisable to revicw pertinent data on loads and to summarize them for the use of designers and building code authorities. The information presented herein consists of results of investigations previously published, much of which is not readily a vailable, and of a recent survey that has provided many new data.

There appear to be several reasons why relatively little work has been done in the past in the way of checking up the weight of building contents. Values given in handbooks, although of uncertain origin in some instances, have been in good agreement and have been generally assumed to represent a reasonable approximation of probable loads. The expense and trouble associated with actually weighing the contents of buildings have deterred efforts to obtain more comprehensive information. Relatively few cases of building collapse due to incorrect load assumptions have occurred, and so there has been no compelling reason for intensive work on load determinations.

Although there has been general acceptance of

*Supervising Structural Engineer, Public Buildings Service, Gencral Scrv ices Administration. conventional load values, investigations from time to time by interested persons have been marle that have thrown some light on the accuracy of common load assumptions. Most of these have been concerned with office buildings. $[1,2]{ }^{1}$

An early study of a vailable data was that made by the Departnient of Commerce Building Code Committee, which prepared a report on recommended live-load assumptions, published by the Bureau in 1925 [3]. The report, now out of print, makes reference to information on loads found in a variety of occupancies, including dwellings, hotels, and other residential occupancies, hospitals, schools, office buildings, library stack rooms, manufacturing buildings of different kinds, and packing plants. Data on densely crowded groups of persons, as in elevators, are also given.

With reference to the loads in manufacturing buildings, the committee observed that the data were regrettably scant but were all that could be obtained from an earnest appeal to the architectural and engineering professions.

Although during the next quarter century, practices changed to some extent in the loading of some occupancies, such as offices and storerooms, little information was published giring the results of actual surveys. However, results of an investigation of loads in two Federal office buildings undertaken by the Public Buildings Adninistration were published in 1946 [4]. One building, the Internal Revenue Building in Washington, D. C., proved to have floor loads of $40 \mathrm{lb} / \mathrm{ft}^{2}$ or less in 88 percent of its area. Certain areas were found to be much more heavily loaded, the maximum average live load of $106 \mathrm{lb} / \mathrm{ft}^{2}$ occurring on $825 \mathrm{ft}^{2}$ of the second floor. In the other case, the Teterans' Administration Building in Tashington, D. C., $97^{3 / 4}$ percent of the area carried an arerage live load of $40 \mathrm{lb} / \mathrm{ft}^{2}$ or less, but there was a maximum average live load on $1,176 \mathrm{ft}^{2}$ of $90 \mathrm{lb} / \mathrm{ft}^{2}$.

In 1945, when the National Bureau of Standards published a report containing the recommendations of the Sectional Committee on Building Code Requirements for Minimum Design Loads in Buildings-A5s, of the American Standards

\footnotetext{
1 Figures in brackets tefer to references at the end of this report.
} 
Association [5], no new information obtained in survers was presented and recommended minimum design values were about the same as in the previous document, although there was one notable exception in the case of the recommended assumption for office loads, which was increased from $50 \mathrm{lb} / \mathrm{ft}^{2}$ in the case of the previous recommendation to $80 \mathrm{lb} / \mathrm{ft}^{2}$ in the new report.

There were several reasons for this changed recommendation. An impression had been obtained by some of the committee members that office building loads were increasing, partly because of more intensive use of office space than formerly and partly because of the growing use of mechanical devices for calculating and other purposes. Facilities were not available for field investigation at that time, but a questionnaire was addressed to building managers asking for their views. The results were inconclusive but a number of replies indicating the possibility of loads greater than $50 \mathrm{lb} / \mathrm{ft}^{2}$ was one factor in influencing the committee's judgment. Another, and perhaps more important development, was the introduction of a new method of providing for reduction of floor live loads in accordance with the probability of loading over extensive areas that permitted allowance for possible concentrations over limited areas and for a rather rapid reduction in design live load as the area concerned increased.

In 1947, the Office of Technical Services in the Department of Commerce sponsored a number of investigations intended to assist in the solution of various business and industrial problems. Among. the subjects selected was an investigation of the weights of combustible contents in various occupancies. Accurate knowledge of such weights is important in establishing the potential fire sererity of such occupancies. When plans for this work were under way, it was pointed out that the total weight of the contents was also of interest because of its relation to building design and that this weight could be readily ascertained by a very slight addition to the program. Accordingly, the work was planned to include the total live load in selected areas. The National Bureau of Standards undertook responsibility for the program and arranged to have the field work done by the Public Building Administration (now the Public Buildings Service) because of the long experience of that organization in the design and management of buildings. Reports of the results of the survey constitute the principal part of this publication.

Necessarily, the survey was somewhat limited because the amount of work involved in such an undertaking is much greater than is generally reaiized, and the funds were sufficient to include only a few buildings. Nerertheless, the work was performed systematically and provided an amount of detail that threw much light on floor loadings in typical occupancies, including the extent to which such loadings varied on different parts of the same floor.

Building codes require that all buildings shall be designed to carry their loarls safely, and gire a list of minimum assumed unit live loads for the more common occupancies. Actual loading of these occupancies may differ from the values given, but it is probable that in ordinary practice most buildings are designed at the minimum ralues. It thus becomes important that these values shall represent the worst conditions for which it is reasonable to provide. For occupancies not giren, values used must be approved by the building department.

In the presentation of results of surveys and other information that follows, an arrangement by broad groups, such as is used in building codes, is employed. Within these groups, the minimum unit live loads usually found in building codes for typical occupancies in the groups, or in some cases a range of such loads, are given for comparison with unit live loads found in the surveys. The maximum unit live loads in the surveys are of principal interest. It should be remembered, however, that even these may not represent the heaviest conditions of loading, since there may be load concentrations not evident in the unit figures which are based on average conditions over rather large areas.

\section{Residential Occupancy}

The permissible minimum live load for purposes of design given in most building codes for residential occupancy is $40 \mathrm{lb} / \mathrm{ft}^{2}$. There are occasional instances where codes permit $30 \mathrm{lb} / \mathrm{ft}^{2}$ on upper floors of single-family dwellings, and the same figure has been adrocated by some authorities for general use throughout dwellings.

Various reasons have been adranced for the selection of $40 \mathrm{lb} / \mathrm{ft}^{2}$ for residential occupancy. Some authorities have pointed out that it takes care of maximum possible loading when persons are assembled at teas, funerals, and other occasions. Other authorities believe that the figure was not intended to represent the actual loads in a dwelling but was selected because a wood-joist floor designed for a lesser load was generally considered too limber for the comfort of the occupants. From the latter point of view, the use of $40 \mathrm{lb} / \mathrm{ft}^{2}$ is thus an indirect method of obtaining desired rigidity in wood-joisted construction.

Although there have been numerous estimates and assumptions made as to live loads in residential occupancy, no published results of figures obtained from weighing the contents of dwellings have been found. Figures for combustible contents given in BMS92, Fire-Resistance Classificentions of Building Constructions, probably approximate closely the total live loads so far as movablé property is concerned [6]. These figures show a maximum of $7.3 \mathrm{lb} / \mathrm{ft}^{2}$ except in one portion occupied by a library, in which the figure is $10.6 \mathrm{lb} / \mathrm{f} \div$ ? Because the weight of persons is not included, some assumption would have to be made for this and added to the figures given. There is also the pos- 
sibility of additional weight in the form of incombustible furnishings.

In the case of hotel rooms, which come within the general residential group, there is a published figure of $4.1 \mathrm{lb} / \mathrm{ft}^{2}[3]$. 'This applies only to the weight of furmiture, however, and therefore does not represent the complete live load.

\section{Business Occupancy}

The permissible minimum live load for purposes of design given in building codes for business occupancy varies to some extent. If office space in buildings is taken as an example, the range in building codes recommended by various organizations for national or regional use and in building code standards is from 50 to $80 \mathrm{lb} / \mathrm{ft}^{2}$.

More attention has been given to loading in office buildings than in any other occupancy. The results of early surveys are described in detail in the following extract from a report of the Department of Commerce Building Code Committee [3].

Offices-The information available on this occupancy is much more complete than for any other. It has been carefully presented in recent technical periodicals and only a resume sufficient to support the committee's recommendations is included here.

Actual weights of furniture and occupants on three complete floors and in a number of selected heavy occupancies in the Equitable Building, New York, N. Y. are reported by C. T. Coley, manager of the building, as follows:

Maximum, minimum, and average live loads in Equitable Building

\begin{tabular}{|c|c|c|c|c|}
\hline & Offices & $\begin{array}{l}\text { Maxi- } \\
\text { mum. }\end{array}$ & $\begin{array}{l}\text { Mini- } \\
\text { mum }\end{array}$ & Average \\
\hline & & $L b s . / \int t^{2}$ & $L b s . / f t^{2}$ & $L b s . / \int t^{2}$ \\
\hline $\begin{array}{l}\text { Light-occupancy floor (twen } \\
\text { tieth) }\end{array}$ & 67 & 55.4 & 0.87 & 10. 26 \\
\hline Medium-occupancy floor (thirty & 64 & 30.73 & 3.27 & 10.67 \\
\hline $\begin{array}{l}\text { Heavy-oceupancy floor (elev. } \\
\text { enth) }\end{array}$ & 62 & 33.84 & 5.0 & 13.96 \\
\hline Total and average & 193 & -.... & $\ldots$ & 11.6 \\
\hline $\begin{array}{l}\text { Selected heavy occupaneies } \\
\text { throughout the building }\end{array}$ & 14 & 78.3 & 21.4 & 42.4 \\
\hline
\end{tabular}

The weights given do not include the radiators, which would add approximately 1 pound per square foot for all exterior bays.

The weight of the partitions was not included in the calculations. These, in general, are 3 -inch hollow tile plastered each side, and one which was being removed was found to weigh 30 pouncls per square foot, or approximately 350 pounds per linear foot.

The weight of occupants, taken at 150 pounds per person, is probably high, as most of the occupants are females, and some studies indicate that an average weight of same would not exceed 120 pounds.

Careful sketches of load arrangement prepared by Mr. Coley made it possible to throw some light on the prevailing method of assuming uniformly distributed live loads as a basis for office floor design, and help to indicate what relation such assumptions should bear to actual total loads. Examination of bays for which the live load was more than 25 pounds per square foot showed wide variation in the distribution of such loads. The larger proportion was found, as might be expected, within a zone approximately 3 fect wirk: around the walls, the remainder being distributerl variously in the centers of the rooms. In one or two cases, however, the major portion of the load was located away from the walls and this condition must be provided for by designers. There is also the probability that practically all furniture may be collected in the centril portion of a floor area when occupants are moving, or when decorating or cleaning is in progress.

The sketches show that the heavier loads, such as library shelves and double filing eabinets, are likely to be located away from walls and partitions. 'This is obviously for ease of access, and the same consicleration demands that when total loads por square foot are high they must be quite uniformly distributed.

The heaviest loading discovered was one incidental to office purposes, being made up chiefly of card filing cases, but the stack room of a law library on one floor would have averaged 87 pounds per square foot if the shelves had been completely filled.

Only eight articles of furniture (safes) were found over 2,000 pounds in weight. A number of sectional filing cases and bookcases with contents weighed much more, but these weights were distributed over such a large area they could not be regarded as concentrated. Of 36 safes and safe cabinets, 23 weighed less than 1,000 pounds; 5 between 1,000 and 2,000 pounds; 2 weighed 2,200 pounds; $2,2,360$ pounds; $1,2,800$ pounds; $1,3,000$ pounds; 1, 3,500 pounds, and $1,4,250$ pounds.

As would naturally be expected, the live loads were found to be lighter next to the exterior walls of the building. Single-row filing cases, cabinets, safes, bookeases, and bins are usually located against blank interior walls. Whether by accident or otherwise, the heavier loads were not found where partitions eut up the floor space into small rooms, indicating that allowance may not be necessary both for movable partitions and heavy floor loads. ${ }^{2}$

Several instances were found where two adjacent floor bays supported average loads of 25 pounds or more, but in no case were two adjacent bays found loaded in excess of an average of 40 pounds per square foot.

There are but two or three instances in the floor plans discussed where three or more offices or storerooms meet at the same column, and it is probable that this condition will be found but rarely in buildings designed for a sufficieney of light and ventilation.

An investigation by M. W. MeIntyre of the Union Central Life Insurance Co.'s building in Cincinnati gave quite similar results. All files, desks, etc., were considered as being 100 per cent full or furnished with all necessary accessories. Following are tabulated the results of Captain MeIntyre's investigations

Weight of employees, computed at the rate of 150 pounds each, added from 0.9 to 1.75 pounds per square foot of floor area.

Office live loads in Union Central Life Insurance Building

\begin{tabular}{|c|c|c|c|c|}
\hline & $\begin{array}{l}\text { Number } \\
\text { of } \\
\text { squarc } \\
\text { feet }\end{array}$ & $\begin{array}{l}\text { Number } \\
\text { of picees } \\
\text { of } \\
\text { furniture }\end{array}$ & $\begin{array}{c}\text { Total } \\
\text { weight } \\
\text { of } \\
\text { furniture }\end{array}$ & $\begin{array}{l}\text { Weight of } \\
\text { furniture } \\
\text { per square } \\
\text { foot }\end{array}$ \\
\hline $\begin{array}{l}\text { Section A } \\
\text { Section B } \\
\text { Section } \mathrm{C} \\
\text { Section } \mathrm{D}\end{array}$ & $\begin{array}{r}10,339 \\
9,303 \\
7,348 \\
10,339\end{array}$ & $\begin{array}{l}635 \\
637 \\
273 \\
702\end{array}$ & $\begin{array}{r}\text { Pounds } \\
104,47 \mathrm{~s} \\
27,055 \\
36,306 \\
121,385\end{array}$ & $\begin{array}{r}\text { Pounds } \\
10.05 \\
2.91 \\
4.92 \\
11.74\end{array}$ \\
\hline Average & 9,332 & 561.5 & 72,314 & 7.405 \\
\hline
\end{tabular}

2 Authors' note: Observation of loading under present conditions indieates some exceptions from this statement. 


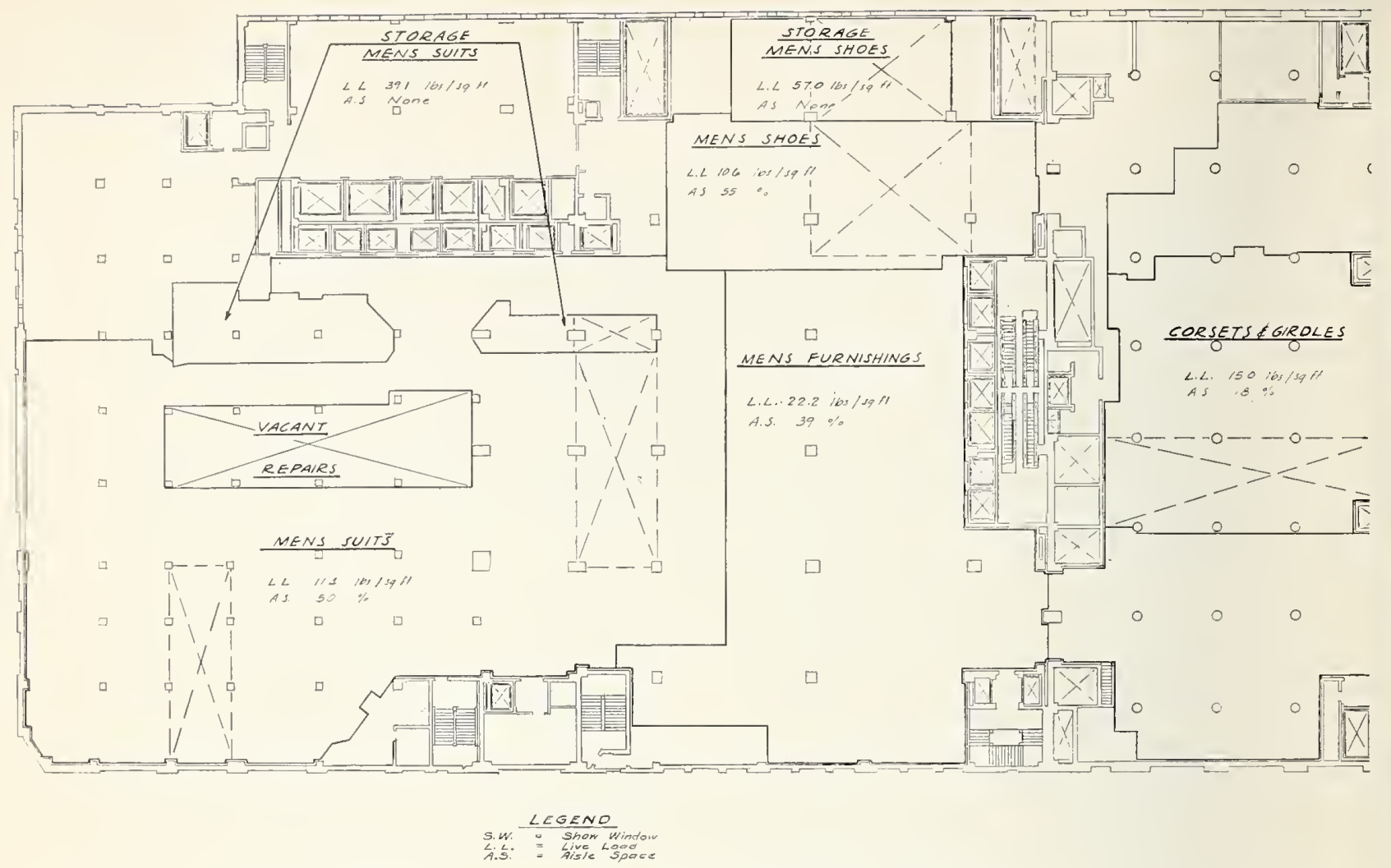

Figure 1. Second-floor plan, department store, New York, N. Y.

Table 1. Live loads in department store, New York, N. Y.Continued

\begin{tabular}{|c|c|c|c|c|c|}
\hline \multirow[b]{2}{*}{ Department } & \multicolumn{3}{|c|}{ Area } & \multicolumn{2}{|c|}{ Unit live load } \\
\hline & $\begin{array}{c}\text { Part } \\
\text { sur- } \\
\text { veyed }\end{array}$ & $\begin{array}{l}\text { Whole } \\
\text { depart- } \\
\text { ment }\end{array}$ & $\begin{array}{l}\text { Aisle } \\
\text { space }\end{array}$ & $\begin{array}{l}\text { As sur- } \\
\text { veyed }\end{array}$ & $\begin{array}{c}\text { With } \\
\text { aisles } \\
\text { crowded s }\end{array}$ \\
\hline \multicolumn{6}{|c|}{ Sixth floor } \\
\hline $\begin{array}{l}\text { Women's shoes } \\
\text { Storage (women's shoes) } \\
\text { Towels. } \\
\text { Blankets } \\
\text { Sheets and linens }\end{array}$ & $\begin{array}{c}f t^{2} \\
1,720 \\
1,100 \\
2,020 \\
1,470 \\
970\end{array}$ & $\begin{array}{r}f t^{2} \\
21,358 \\
12,085 \\
6,882 \\
6,811 \\
17,379\end{array}$ & $\begin{array}{c}\text { Per- } \\
\text { cent } \\
64 \\
0 \\
41 \\
42 \\
39\end{array}$ & $\begin{array}{l}2 b / f t^{2} \\
15.4 \\
42.0 \\
12.8 \\
11.8 \\
20.3\end{array}$ & $\begin{array}{l}2 b / f t^{2} \\
51.8 \\
37.4 \\
37.0 \\
43.7\end{array}$ \\
\hline 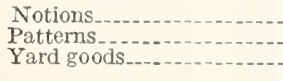 & $\begin{array}{r}1,220 \\
420 \\
1,970 \\
\end{array}$ & $\begin{array}{r}2,238 \\
2,525 \\
26,904 \\
\end{array}$ & $\begin{array}{l}34 \\
61 \\
41\end{array}$ & $\begin{array}{l}14.1 \\
19.9 \\
15.6\end{array}$ & $\begin{array}{l}34.5 \\
58.3 \\
40.2\end{array}$ \\
\hline Total & & 96,182 & & & \\
\hline \multicolumn{6}{|c|}{ Seventh floor } \\
\hline $\begin{array}{l}\text { Linoleum } \\
\text { Rugs } \\
\text { Candles } \\
\text { Lamps and shades } \\
\text { Curtains }\end{array}$ & $\begin{array}{r}810 \\
3,360 \\
200 \\
990 \\
970\end{array}$ & $\begin{array}{r}3,070 \\
27,651 \\
200 \\
7,868 \\
14,430\end{array}$ & $\begin{array}{l}64 \\
44 \\
21 \\
73 \\
67\end{array}$ & $\begin{array}{r}7.5 \\
8.7 \\
32.3 \\
9.4 \\
5.2\end{array}$ & $\begin{array}{l}45.9 \\
35.1 \\
44.9 \\
53.2 \\
45.2\end{array}$ \\
\hline $\begin{array}{l}\text { Closet shop } \\
\text { Walpaper } \\
\text { Assorted yard goods }\end{array}$ & $\begin{array}{l}510 \\
900 \\
(\bullet) \\
\end{array}$ & $\begin{array}{r}8,109 \\
1,190 \\
23,303 \\
\end{array}$ & $\begin{array}{l}78 \\
77 \\
41\end{array}$ & $\begin{array}{l}11.6 \\
10.1 \\
15.6\end{array}$ & $\begin{array}{l}58.4 \\
56.3 \\
40.2\end{array}$ \\
\hline Total & - & 85,821 & & & \\
\hline
\end{tabular}

TABLE 1. Live loads in department store, New York, N. Y.Continued

\begin{tabular}{|c|c|c|c|c|c|}
\hline \multirow[b]{2}{*}{ Department } & \multicolumn{3}{|c|}{ Area } & \multicolumn{2}{|c|}{ Unit live load } \\
\hline & $\begin{array}{l}\text { Part } \\
\text { sur- } \\
\text { veyed }\end{array}$ & $\begin{array}{l}\text { Whole } \\
\text { depart- } \\
\text { ment }\end{array}$ & $\begin{array}{l}\text { Aisle } \\
\text { space }\end{array}$ & $\begin{array}{l}\text { As sur- } \\
\text { veyed }\end{array}$ & $\begin{array}{l}\text { With } \\
\text { aisles } \\
\text { crowded a }\end{array}$ \\
\hline \multicolumn{6}{|c|}{ Eighth floor } \\
\hline $\begin{array}{l}\text { Food } \\
\text { Glassware } \\
\text { Chinaware } \\
\text { Pictures and frames. } \\
\text { Luggage. }\end{array}$ & $\begin{array}{c}f t^{2} \\
1,970 \\
1,730 \\
1,930 \\
1,820 \\
1,930\end{array}$ & \begin{tabular}{r}
\multicolumn{1}{c}{$t^{2}$} \\
10,190 \\
9,438 \\
19,244 \\
7,253 \\
8,149
\end{tabular} & $\begin{array}{c}\text { Per- } \\
\text { cent } \\
63 \\
66 \\
64 \\
69 \\
38\end{array}$ & $\begin{array}{c}l b / f t^{2} \\
16.7 \\
10.6 \\
14.7 \\
7.0 \\
7.4\end{array}$ & $\begin{array}{l}l b / f t t^{2} \\
54.5 \\
50.2 \\
53.1 \\
48.4 \\
30.2\end{array}$ \\
\hline Total & -...-. & 54,274 & $-\ldots$ & -...... & $\ldots$ \\
\hline \multicolumn{6}{|c|}{ Ninth floor } \\
\hline $\begin{array}{l}\text { Bedroom furniture } \\
\text { Dining room and occa. } \\
\text { sional furniture } \\
\text { Modern furniture... }\end{array}$ & $\begin{array}{l}3,960 \\
2,680 \\
2,180\end{array}$ & $\begin{array}{l}24,929 \\
55,847 \\
12,513\end{array}$ & $\begin{array}{l}53 \\
39 \\
34\end{array}$ & $\begin{array}{r}4.8 \\
4.1 \\
13.2\end{array}$ & $\begin{array}{l}36.6 \\
27.5 \\
33.6\end{array}$ \\
\hline Total & -......- & 93,289 & $\ldots$ & $\ldots$ & - ......... \\
\hline Grand total $\ldots$ & $\ldots$ & 790,793 & - & $-\cdots$ & - \\
\hline
\end{tabular}

s At rate of $60 \mathrm{lb} / \mathrm{ft}^{2}$ additional loading for aisle space.

b See similar department on first floor.

- See similar department on third floor.

d See similar department on this floor. 


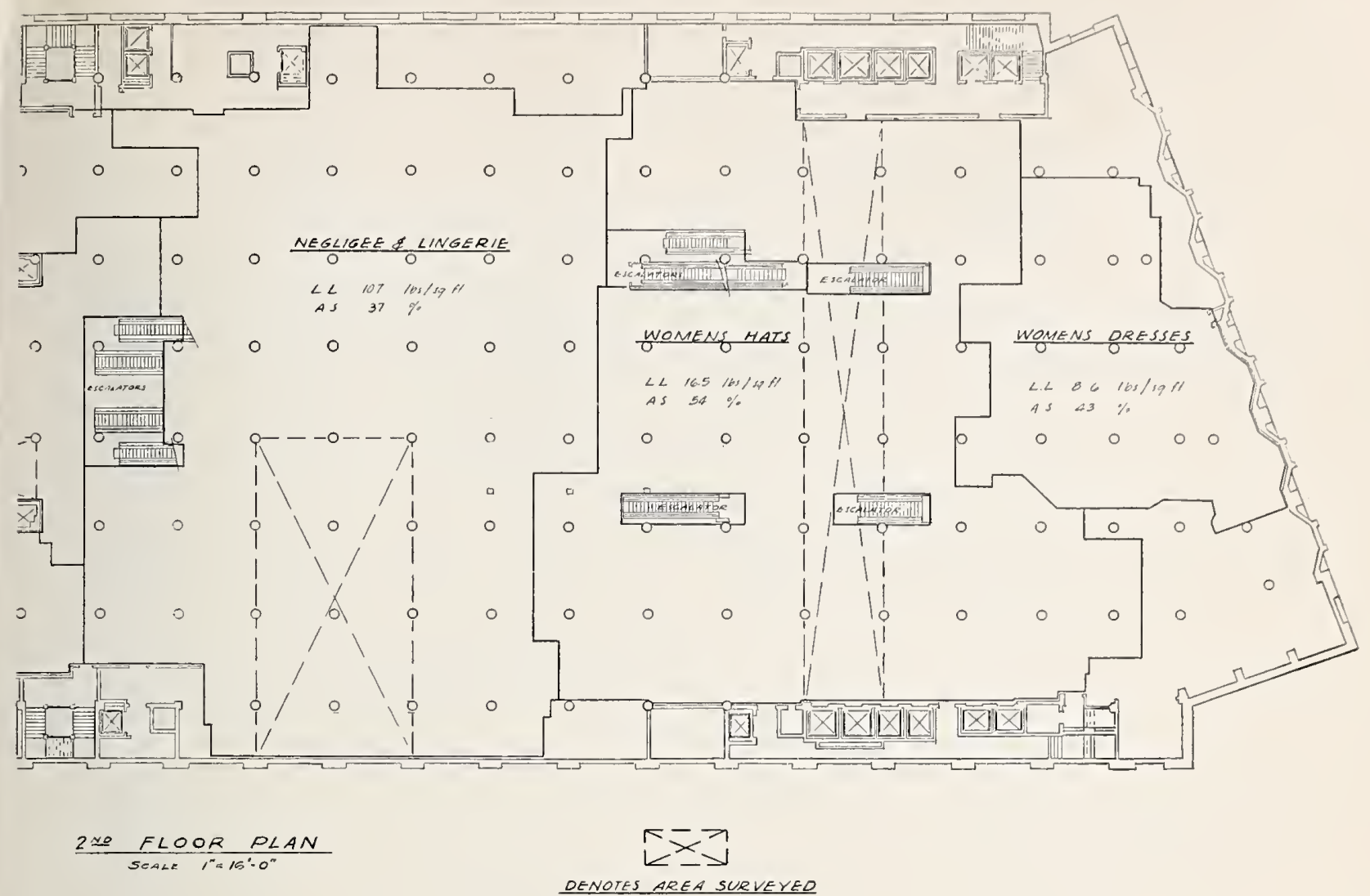

Figure 1-Continued

TABLE 2. Tariation in live loads in department store, New York, $N$. Y.

\begin{tabular}{|c|c|c|}
\hline Unit load (lb/ft ${ }^{2}$ ) & Area & $\begin{array}{l}\text { Portion of } \\
\text { total area }\end{array}$ \\
\hline $\begin{array}{l}20.0 \text { to } 24.9 \\
25.0 \text { to } 29.9 \\
30.0 \text { to } 34.9 \\
40.0 \text { to } 39.9\end{array}$ & $\begin{array}{c}f t^{2} \\
21,282 \\
74,527 \\
111,442 \\
119,305 \\
198,883\end{array}$ & $\begin{array}{c}\text { Percent } \\
2.7 \\
9.4 \\
14.1 \\
15.1 \\
25.1\end{array}$ \\
\hline $\begin{array}{l}45.0 \text { to } 49.9 \\
50.0 \text { to } 54.9 \\
55.0 \text { to } 59.9 \\
60.0 \text { to } 64.9\end{array}$ & $\begin{array}{r}148,463 \\
86,688 \\
23,803 \\
6,400\end{array}$ & $\begin{array}{r}18.8 \\
11.0 \\
3.0 \\
0.8\end{array}$ \\
\hline Total & 790,793 & 100.0 \\
\hline
\end{tabular}

TABLE 3. Live loads in department store, Washington, D. C

\begin{tabular}{|c|c|c|c|c|}
\hline \multirow[b]{2}{*}{ Department } & \multicolumn{2}{|c|}{ Area : } & \multicolumn{2}{|c|}{ Unit live load } \\
\hline & $\begin{array}{l}\text { Whole } \\
\text { depart- } \\
\text { meut }\end{array}$ & $\begin{array}{l}\text { A isle } \\
\text { spacc }\end{array}$ & $\begin{array}{l}\text { Assur- } \\
\text { vesyed }\end{array}$ & $\begin{array}{l}\text { With aisles } \\
\text { crowded b }\end{array}$ \\
\hline \multicolumn{5}{|c|}{ First floor } \\
\hline $\begin{array}{l}\text { Dry cleaning counter... } \\
\text { Books } \\
\text { Candy } \\
\text { Notions-. } \\
\text { Umbrellas }\end{array}$ & $\begin{array}{r}f t^{2} \\
320 \\
3,450 \\
1,600 \\
9,750 \\
350\end{array}$ & $\begin{array}{c}\text { Percent } \\
37 \\
28 \\
29 \\
43 \\
20\end{array}$ & $\begin{array}{l}7 b / f t^{2} \\
15.1 \\
21.4 \\
16.5 \\
17.8 \\
26.3\end{array}$ & $\begin{array}{l}7 b / f t^{z} \\
37.3 \\
38.2 \\
33.9 \\
43.6 \\
38.6\end{array}$ \\
\hline $\begin{array}{l}\text { Service } \\
\text { Cosmetics } \\
\text { Handbags and leather goods } \\
\text { Hat bar } \\
\text { Stationery }\end{array}$ & $\begin{array}{r}150 \\
6,350 \\
2,800 \\
300 \\
5,500\end{array}$ & $\begin{array}{l}60 \\
40 \\
38 \\
27 \\
41\end{array}$ & $\begin{array}{l}30.7 \\
16.6 \\
13.4 \\
17.7 \\
16.0\end{array}$ & $\begin{array}{l}66.7 \\
40.6 \\
36.2 \\
33.9 \\
40.6\end{array}$ \\
\hline $\begin{array}{l}\text { Costume jewelry } \\
\text { Jewelry }\end{array}$ & $\begin{array}{l}2,900 \\
3,350\end{array}$ & $\begin{array}{l}3 \overrightarrow{7} \\
36\end{array}$ & $\begin{array}{l}13.2 \\
16.7\end{array}$ & $\begin{array}{l}35.4 \\
38.3\end{array}$ \\
\hline Total ......... & 36,820 & .... & $\cdots$ & - n-n- \\
\hline \multicolumn{5}{|c|}{ Second floor } \\
\hline Drygoods, patterns, and art & 8,752 & 32 & & 28.9 \\
\hline $\begin{array}{l}\text { Ladies' shoe stock room } \\
\text { Children's shoe stock room }\end{array}$ & $\begin{array}{r}2,000 \\
955\end{array}$ & $\begin{array}{l}30 \\
20\end{array}$ & $\begin{array}{l}32.8 \\
28.1\end{array}$ & - \\
\hline Shoc salc space- & 3,944 & 35 & 5.8 & 26.1 \\
\hline $\begin{array}{l}\text { Storage (men's hats, shocs, } \\
\text { tobacco, etc.) } \\
\text { Men's clothing }\end{array}$ & $\begin{array}{r}809 \\
16,227\end{array}$ & $\begin{array}{l}20 \\
50\end{array}$ & $\begin{array}{l}32.2 \\
15.1\end{array}$ & 45.1 \\
\hline Total_ & 32,687 & --- & $---\cdot$ & - - - - \\
\hline
\end{tabular}

See footnotes at end of table. 


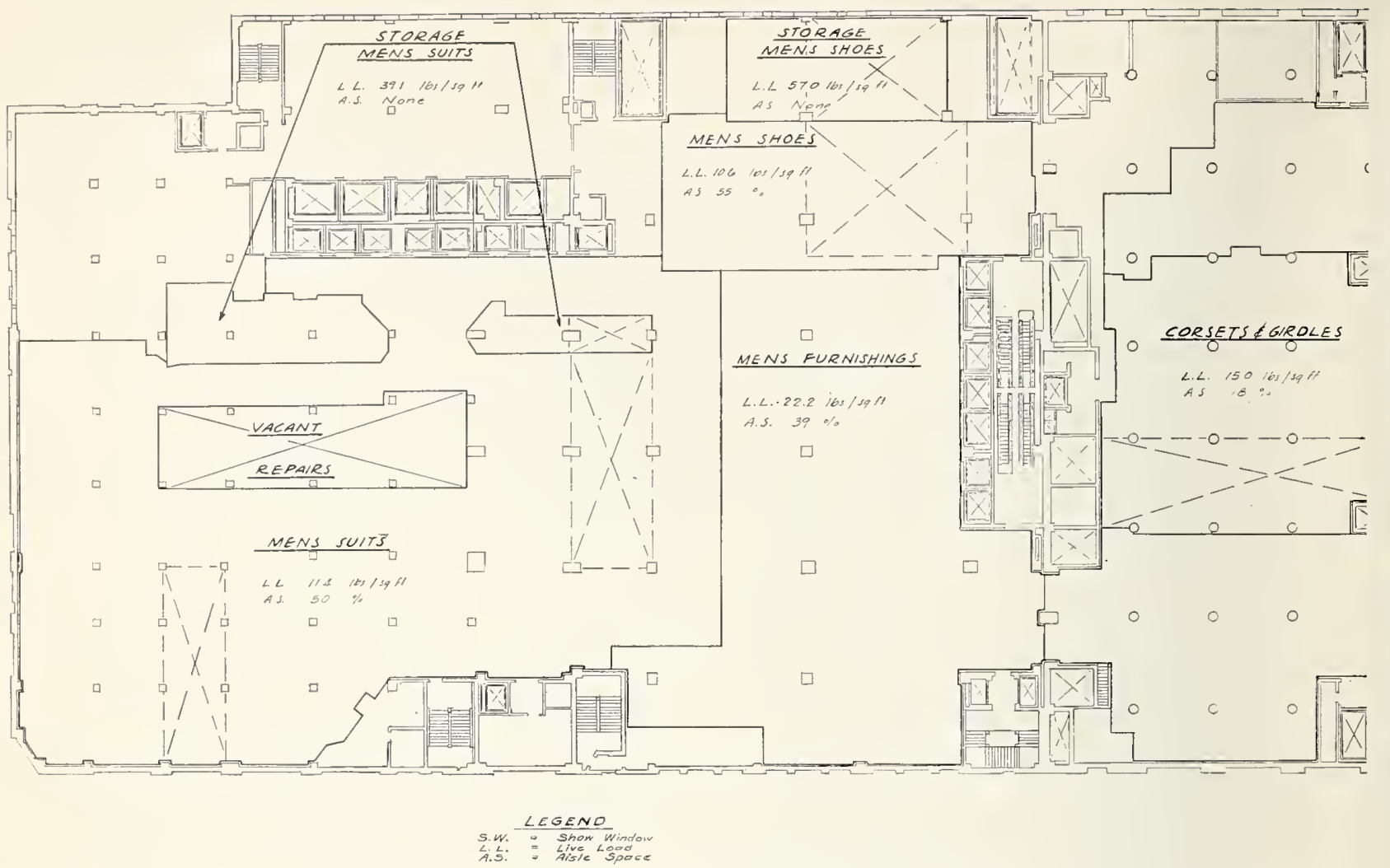

Figure 1. Second-floor plan, department store, New York, N. Y.

TABLE 1. Live loads in department store, New York, N. Y.Continued

\begin{tabular}{|c|c|c|c|c|c|}
\hline \multirow[b]{2}{*}{$d$ Department } & \multicolumn{3}{|c|}{ Area } & \multicolumn{2}{|c|}{ Unit live load } \\
\hline & $\begin{array}{c}\text { Part } \\
\text { sur- } \\
\text { veyed }\end{array}$ & $\begin{array}{l}\text { Whole } \\
\text { depart- } \\
\text { ment }\end{array}$ & $\begin{array}{l}\text { Aisle } \\
\text { space }\end{array}$ & $\begin{array}{l}\text { As sur- } \\
\text { veyed }\end{array}$ & $\begin{array}{l}\text { With } \\
\text { aisles } \\
\text { crowded a }\end{array}$ \\
\hline \multicolumn{6}{|c|}{ Sixth floor } \\
\hline $\begin{array}{l}\text { Women's shoes_- } \\
\text { Storage (women's shoes) } \\
\text { Towels. } \\
\text { Blankets.- } \\
\text { Sheets and linens }\end{array}$ & $\begin{array}{c}f t^{2} \\
1,720 \\
1,100 \\
2,020 \\
1,470 \\
970\end{array}$ & $\begin{array}{r}f t^{2} \\
21,358 \\
12,085 \\
6,882 \\
6,811 \\
17,379\end{array}$ & $\begin{array}{c}P_{e r-} \\
\text { cent } \\
64 \\
0 \\
41 \\
42 \\
39\end{array}$ & $\begin{array}{l}l b / f t^{2} \\
15.4 \\
42.0 \\
12.8 \\
11.8 \\
20.3\end{array}$ & $\begin{array}{l}l b / f t 2 \\
51.8 \\
37.4 \\
37.0 \\
43.7\end{array}$ \\
\hline $\begin{array}{l}\text { Notions } \\
\text { Patterns }-.- \\
\text { Yard goods.-..- }\end{array}$ & $\begin{array}{r}1,220 \\
420 \\
1,970\end{array}$ & $\begin{array}{r}2,238 \\
2,525 \\
26,904\end{array}$ & $\begin{array}{l}34 \\
64 \\
41\end{array}$ & $\begin{array}{l}\text { 14. } 1 \\
19.9 \\
15.6\end{array}$ & $\begin{array}{l}34.5 \\
58.3 \\
40.2\end{array}$ \\
\hline Total & - & 96,182 & & $\ldots$ & .... \\
\hline \multicolumn{6}{|c|}{ Seventh floor } \\
\hline $\begin{array}{l}\text { Linoleum } \\
\text { Rugs } \\
\text { Candles } \\
\text { Lamps and shades } \\
\text { Curtains. }\end{array}$ & $\begin{array}{r}810 \\
3,360 \\
200 \\
990 \\
970\end{array}$ & $\begin{array}{r}3,070 \\
27,651 \\
200 \\
7,868 \\
14,430\end{array}$ & $\begin{array}{l}64 \\
44 \\
21 \\
73 \\
67\end{array}$ & $\begin{array}{r}7.5 \\
8.7 \\
32.3 \\
9.4 \\
5.2\end{array}$ & $\begin{array}{l}45.9 \\
35.1 \\
44.9 \\
53.2 \\
45.2\end{array}$ \\
\hline $\begin{array}{l}\text { Closet shop } \\
\text { Wallpaper-ard } \\
\text { Assorted yard goods }\end{array}$ & $\begin{array}{c}510 \\
900 \\
(e)\end{array}$ & $\begin{array}{r}8,109 \\
1,190 \\
23,303\end{array}$ & $\begin{array}{l}78 \\
77 \\
41\end{array}$ & $\begin{array}{l}11.6 \\
10.1 \\
15.6\end{array}$ & $\begin{array}{l}58.4 \\
56.3 \\
40.2\end{array}$ \\
\hline Total_ & & 85,821 & & & \\
\hline
\end{tabular}

Table 1. Live loads in department store, New York, N. Y.Continued

\begin{tabular}{|c|c|c|c|c|c|}
\hline \multirow[b]{2}{*}{ Department } & \multicolumn{3}{|c|}{ Area } & \multicolumn{2}{|c|}{ Unit live load } \\
\hline & $\begin{array}{l}\text { Part } \\
\text { sur- } \\
\text { veyed }\end{array}$ & $\begin{array}{l}\text { Whole } \\
\text { depart- } \\
\text { ment }\end{array}$ & $\begin{array}{c}\text { Aisle } \\
\text { space }\end{array}$ & $\begin{array}{l}\text { As sur- } \\
\text { veyed }\end{array}$ & $\begin{array}{c}\text { With } \\
\text { aisles } \\
\text { crowded a }\end{array}$ \\
\hline \multicolumn{6}{|c|}{ Eighth flool } \\
\hline $\begin{array}{l}\text { Food } \\
\text { Glassware } \\
\text { Chinaware } \\
\text { Pictures and frames. } \\
\text { Luggage. }\end{array}$ & $\begin{array}{c}f t^{2} \\
1,970 \\
1,730 \\
1,930 \\
1,820 \\
1,930\end{array}$ & $\begin{array}{r}f t^{2} \\
10,190 \\
9,438 \\
19,244 \\
7,253 \\
8,149\end{array}$ & $\begin{array}{c}\text { Per- } \\
\text { cent } \\
63 \\
66 \\
64 \\
69 \\
38\end{array}$ & $\begin{array}{c}l b / f t^{2} \\
16.7 \\
10.6 \\
14.7 \\
7.0 \\
7.4\end{array}$ & $\begin{array}{l}l b / f t^{2} \\
54.5 \\
50.2 \\
53.1 \\
48.4 \\
30.2\end{array}$ \\
\hline Total & 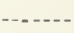 & 54,274 & & & - - - \\
\hline \multicolumn{6}{|c|}{ Ninth floor } \\
\hline $\begin{array}{l}\text { Bedroom furniture } \\
\text { Dining room and ocea- } \\
\text { sional fumiture } \\
\text { Modern furmiture........ }\end{array}$ & $\begin{array}{l}3,960 \\
2,680 \\
2,180\end{array}$ & $\begin{array}{l}24,929 \\
55,847 \\
12,513\end{array}$ & $\begin{array}{l}53 \\
39 \\
34\end{array}$ & $\begin{array}{r}4.8 \\
4.1 \\
13.2\end{array}$ & $\begin{array}{l}36.6 \\
27.5 \\
33.6\end{array}$ \\
\hline Total & -....-. & 93,289 & $-\ldots \ldots$ & - $\ldots$ & -... \\
\hline Grand total & $-\ldots$ & 790,793 & -- & $-\cdots$ & - \\
\hline
\end{tabular}

a At rate of $60 \mathrm{lb} / \mathrm{ft}{ }^{2}$ additional loading for aisle space,

b See similar department on first floor.

- See similar department on third floor.

d See similar department on this floor. 


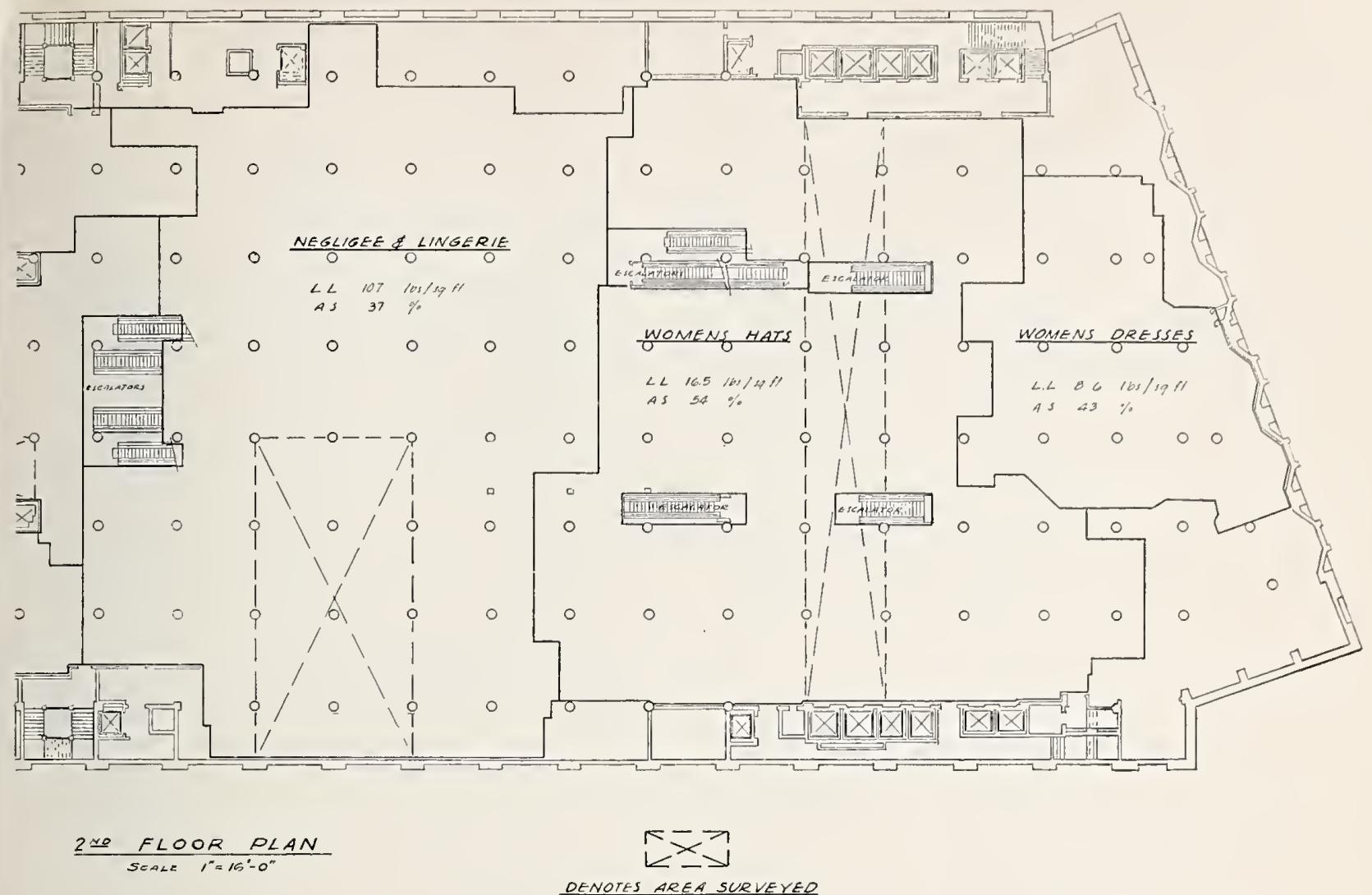

Figure 1-Continued

TABLE 2. Tariation in live loads in department store, New York, N. Y.

\begin{tabular}{|c|c|c|}
\hline Unit load (lb/ft $\left.{ }^{2}\right)$ & Area & $\begin{array}{l}\text { Portion of } \\
\text { total area }\end{array}$ \\
\hline $\begin{array}{l}20.0 \text { to } 24.9 \\
25.0 \text { to } 29.9 \\
30.0 \text { to } 34.9 \\
35.0 \text { to } 39.9 \\
40.0 \text { to } 44.9\end{array}$ & $\begin{array}{c}f t^{2} \\
21,282 \\
74,527 \\
111,442 \\
119,305 \\
198,883\end{array}$ & $\begin{array}{c}\text { Percent } \\
2.7 \\
9.4 \\
14.1 \\
15.1 \\
25.1\end{array}$ \\
\hline $\begin{array}{l}45.0 \text { to } 49.9 \\
50.0 \text { to } 54.9 \\
55.0 \text { to } 59.9 \\
60.0 \text { to } 64.9\end{array}$ & $\begin{array}{r}148,463 \\
86,688 \\
23,803 \\
6,400\end{array}$ & $\begin{array}{r}18.8 \\
11.0 \\
3.0 \\
0.8\end{array}$ \\
\hline Total & 790,793 & 100.0 \\
\hline
\end{tabular}

TABLE 3. Live loads in department store, Washington, D. C

\begin{tabular}{|c|c|c|c|c|}
\hline \multirow[b]{2}{*}{ Department } & \multicolumn{2}{|c|}{ Area s } & \multicolumn{2}{|c|}{ Unit live load } \\
\hline & $\begin{array}{l}\text { Whole } \\
\text { depart- } \\
\text { ment }\end{array}$ & $\begin{array}{l}\text { Aisle } \\
\text { space }\end{array}$ & $\begin{array}{l}\text { Assur- } \\
\text { veyed }\end{array}$ & $\begin{array}{l}\text { With aisles } \\
\text { crowded b }\end{array}$ \\
\hline \multicolumn{5}{|c|}{ Fir'st floor } \\
\hline $\begin{array}{l}\text { Dry cleaning counter } \\
\text { Books } \\
\text { Candy } \\
\text { Notions-.-. } \\
\text { Umbrellas.-. }\end{array}$ & $\begin{array}{l}f t^{2} \\
320 \\
3,450 \\
1,600 \\
9,750 \\
350\end{array}$ & $\left\{\begin{array}{c}\text { Percent } \\
37 \\
28 \\
29 \\
43 \\
20\end{array}\right.$ & $\begin{array}{l}2 b / f t^{2} \\
15.1 \\
21.4 \\
16.5 \\
17.8 \\
26.3\end{array}$ & $\begin{array}{l}l b l f t^{3} \\
37.3 \\
38.2 \\
3.9 \\
43.6 \\
38.3\end{array}$ \\
\hline $\begin{array}{l}\text { Scrvice } \\
\text { Cosmetics } \\
\text { Handbags and leather goods } \\
\text { Hat bar } \\
\text { Stationery }\end{array}$ & $\begin{array}{r}150 \\
6,350 \\
2,800 \\
300 \\
5,500\end{array}$ & $\begin{array}{l}60 \\
40 \\
35 \\
27 \\
41\end{array}$ & $\begin{array}{l}30.7 \\
16.6 \\
13.4 \\
17.7 \\
16.0\end{array}$ & $\begin{array}{l}66.7 \\
40.6 \\
36.2 \\
33.9 \\
40.6\end{array}$ \\
\hline $\begin{array}{l}\text { Costume jewelry } \\
\text { Jewelry }\end{array}$ & $\begin{array}{l}2,900 \\
3,350\end{array}$ & $\begin{array}{l}37 \\
36\end{array}$ & $\begin{array}{l}13.2 \\
16.7\end{array}$ & $\begin{array}{l}35.4 \\
38.3\end{array}$ \\
\hline Total. . & 36,820 & $\cdots-$ & -... & - - - n-- \\
\hline \multicolumn{5}{|c|}{ Second floor } \\
\hline $\begin{array}{l}\text { Drygoods, patterns, and art } \\
\text { goods }\end{array}$ & 8.752 & 32 & 9.7 & 28.9 \\
\hline Ladies' sboe stock room & 2,000 & 30 & 32.8 & -.....-- \\
\hline $\begin{array}{l}\text { Cbildren's shoe stock room } \\
\text { Shoe sale space }\end{array}$ & $\begin{array}{r}955 \\
3,941\end{array}$ & 35 & 5.8 & 26.1 \\
\hline $\begin{array}{l}\text { Storage (men's bats, shoes, } \\
\text { tobacco, etc.) }\end{array}$ & 809 & 20 & 32.2 & \\
\hline Men's clothing & 16,227 & 50 & 15.1 & 45.1 \\
\hline Total & 32,687 & --- & -..- & --_--... \\
\hline
\end{tabular}

See footnotes at end of table. 
TABLE 3. Live loads in department store, Washington. D. C.-Continued

\begin{tabular}{|c|c|c|c|c|}
\hline \multirow[b]{2}{*}{ Department } & \multicolumn{2}{|c|}{ Area a } & \multicolumn{2}{|c|}{ Unit live load } \\
\hline & $\begin{array}{l}\text { Whok } \\
\text { depart- } \\
\text { ment }\end{array}$ & $\begin{array}{l}\text { Aisle } \\
\text { spaee }\end{array}$ & $\begin{array}{l}\text { Assur- } \\
\text { veyed }\end{array}$ & $\begin{array}{l}\text { With aisles } \\
\text { erowded b }\end{array}$ \\
\hline \multicolumn{5}{|c|}{ Third floor } \\
\hline $\begin{array}{l}\text { Women's hats } \\
\text { Lingerie and dresses. } \\
\text { Ladies' ready-to-wear..... } \\
\text { Ladies' gowns and furs.... }\end{array}$ & $\begin{array}{r}f t^{2} \\
4,300 \\
5,700 \\
16,350 \\
18,500\end{array}$ & $\begin{array}{c}\text { Percent } \\
50 \\
40 \\
45 \\
53\end{array}$ & $\begin{array}{r}l b / f t^{2} \\
8.7 \\
13.0 \\
6.7 \\
6.5\end{array}$ & $\begin{array}{l}l b / f t^{2} \\
38.7 \\
37.0 \\
33.7 \\
38.3\end{array}$ \\
\hline Total $\ldots$ & 44,850 & ...- & $\ldots$ & -........- \\
\hline \multicolumn{5}{|c|}{ Fourth floor } \\
\hline $\begin{array}{l}\text { Boys' elothing } \\
\text { Infant and juvenile elothing } \\
\text { Camera and radio } \\
\text { Musie. } \\
\text { Junior misses............. }\end{array}$ & $\begin{array}{r}3,830 \\
11,934 \\
3,958 \\
2,870 \\
14,022\end{array}$ & $\begin{array}{l}33 \\
40 \\
30 \\
50 \\
60\end{array}$ & $\begin{array}{r}15.9 \\
8.8 \\
12.0 \\
25.0 \\
5.6\end{array}$ & $\begin{array}{l}35.7 \\
32.8 \\
30.0 \\
55.0 \\
41.6\end{array}$ \\
\hline Total & 36,614 & -..- & -..- & -....... \\
\hline
\end{tabular}

Fifth floor

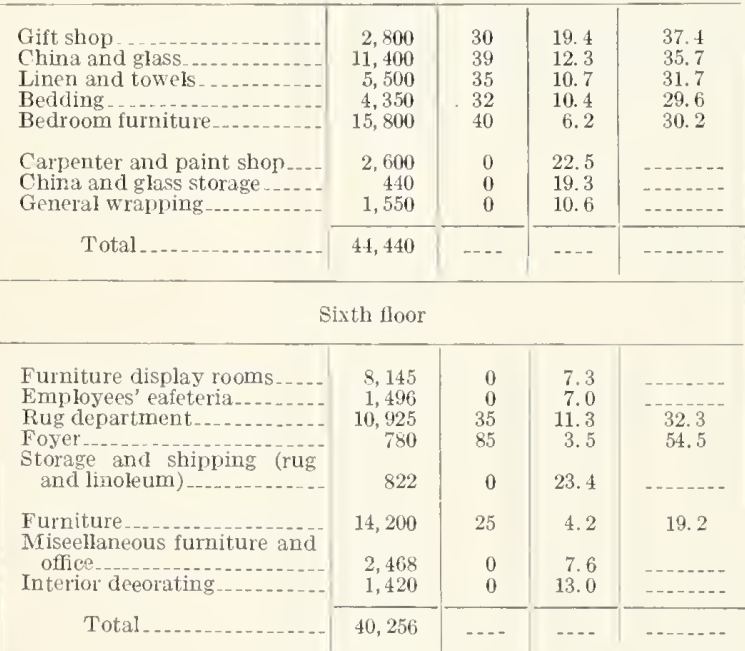

Seventh floor

\begin{tabular}{|c|c|c|c|c|}
\hline $\begin{array}{l}\text { Luggage } \\
\text { Pietures } \\
\text { Lamps } \\
\text { Draperies... } \\
\text { Drapery storage No. } 1 . . .\end{array}$ & $\begin{array}{l}4,250 \\
2,100 \\
4,100 \\
9,000 \\
960\end{array}$ & $\begin{array}{r}39 \\
38 \\
65 \\
46 \\
0\end{array}$ & $\begin{array}{r}6.0 \\
15.8 \\
9.7 \\
8.7 \\
23.6\end{array}$ & $\begin{array}{l}29.4 \\
38.6 \\
48.7 \\
36.3 \\
- \\
-\end{array}$ \\
\hline $\begin{array}{l}\text { Drapery storage No. } 2 . \\
\text { Auditorium } \\
\text { Offiees } \\
\text { Waiting room at tearoom } \\
\text { Fountain room and foumtain }\end{array}$ & $\begin{array}{r}550 \\
800 \\
4,000 \\
1,200 \\
2,900\end{array}$ & $\begin{array}{r}0 \\
-0 \\
100 \\
0\end{array}$ & $\begin{array}{r}42.8 \\
5.4 \\
10.7 \\
1.6 \\
11.9\end{array}$ & $\begin{array}{l}10.7 \\
61.6\end{array}$ \\
\hline $\begin{array}{l}\text { Tearoom } \\
\text { Linen storage (tearoom) }\end{array}$ & $\begin{array}{r}8,300 \\
380\end{array}$ & $\begin{array}{l}0 \\
0\end{array}$ & $\begin{array}{r}8.6 \\
30.2\end{array}$ & \\
\hline Total & 38,540 & & -- & \\
\hline
\end{tabular}

Taвle 3. Live loads in department store, Washington D. C.-Continued

\begin{tabular}{|c|c|c|c|c|}
\hline \multirow[b]{2}{*}{ Department } & \multicolumn{2}{|c|}{ Area a } & \multicolumn{2}{|c|}{ Unit live load } \\
\hline & $\begin{array}{l}\text { Whole } \\
\text { depart- } \\
\text { ment }\end{array}$ & $\begin{array}{l}\text { Aisle } \\
\text { spaee }\end{array}$ & $\begin{array}{l}\text { As sur- } \\
\text { veyed }\end{array}$ & $\begin{array}{l}\text { With aisles } \\
\text { erowded b }\end{array}$ \\
\hline \multicolumn{5}{|c|}{ Eighth floor } \\
\hline $\begin{array}{l}\text { Paint } \\
\text { Household goods } \\
\text { Groeeries } \\
\text { Cold storage for groeeries } \\
\text { Refrigerators, ete }\end{array}$ & $\begin{array}{l}f t^{2} \\
1,749 \\
7,781 \\
3,500 \\
400 \\
2,307\end{array}$ & $\begin{array}{c}\text { Percent } \\
24 \\
30 \\
37 \\
0 \\
23\end{array}$ & $\begin{array}{c}l b / f t^{2} \\
22.6 \\
7.9 \\
8.3 \\
24.1 \\
15.0\end{array}$ & $\begin{array}{c}l b / f t^{2} \\
37.0 \\
25.9 \\
30.5 \\
28.8\end{array}$ \\
\hline $\begin{array}{l}\text { Electrieal } \\
\text { Bathroom fittings. } \\
\text { Cafeteria } \\
\text { Central wrapping } \\
\text { Bakery }\end{array}$ & $\begin{array}{l}1,400 \\
3,205 \\
3,834 \\
2,556 \\
3,210\end{array}$ & $\begin{array}{r}50 \\
46 \\
0 \\
0 \\
0\end{array}$ & $\begin{array}{r}12.9 \\
9.0 \\
4.9 \\
11.3 \\
10.9\end{array}$ & $\begin{array}{c}42.9 \\
36.6 \\
-1.2- \\
-0 .-\end{array}$ \\
\hline $\begin{array}{l}\text { Paper storage } \\
\text { Fur fitting } \\
\text { Offiee. }\end{array}$ & $\begin{array}{r}360 \\
2,000 \\
7,230\end{array}$ & $\begin{array}{l}0 \\
0 \\
0\end{array}$ & $\begin{array}{r}24.6 \\
4.1 \\
10.9\end{array}$ & - \\
\hline Total ... & 39,532 & --- & -... & ....... \\
\hline Grand total ..... & 313,739 & $\ldots$ & -... & $\ldots$ \\
\hline
\end{tabular}

a The entire department was survered in eaeh ease.

At rate of $60 \mathrm{lb} / \mathrm{ft}^{2}$ additional loading for aisle spaee.

TABLE 4. T'ariation in live loads in department store, WTashington, $D . C$.

\begin{tabular}{|c|c|c|}
\hline Unit load $\left(1 \mathrm{~b} / \mathrm{ft}^{2}\right)$ & Area & $\begin{array}{l}\text { Portion of } \\
\text { total area }\end{array}$ \\
\hline $\begin{array}{l}0.0 \text { to } 4.9 \\
5.0 \text { to } 9.9 \\
10.0 \text { to } 14.9 \\
15.0 \text { to } 19.9 \\
20.0 \text { to } 24.9\end{array}$ & \begin{tabular}{r}
\multicolumn{1}{r}{$f^{2}$} \\
5,834 \\
21,209 \\
22,866 \\
14,640 \\
5,142
\end{tabular} & $\begin{array}{c}\text { Percent } \\
1.7 \\
6.8 \\
7.3 \\
4.7 \\
1.6\end{array}$ \\
\hline $\begin{array}{l}25.0 \text { to } 29.9 \\
30.0 \text { to } 34.9 \\
35.0 \text { to } 39.9 \\
40.0 \text { to } 44.9 \\
45.0 \text { to } 49.9\end{array}$ & $\begin{array}{l}31,384 \\
54,447 \\
91,554 \\
39,336 \\
20,327\end{array}$ & $\begin{array}{r}10.0 \\
17.4 \\
29.2 \\
12.5 \\
6.5\end{array}$ \\
\hline $\begin{array}{l}50.0 \text { to } 54.9 \\
55.0 \text { to } 59.9 \\
60.0 \text { to } 64.9 \\
65.0 \text { to } 69.9\end{array}$ & $\begin{array}{r}2,780 \\
2,870 \\
1,200 \\
150\end{array}$ & $\begin{array}{r}0.9 \\
.9 \\
.4 \\
.1\end{array}$ \\
\hline Total......... & 313,739 & 100.0 \\
\hline
\end{tabular}

\section{Assembly Occupancy}

"Assembly occupancy" includes theaters, dance halls, auditoriums, churches, and schools.

Building codes recommended by various organizations for national or regional ise and building code standards give permissible minimum live loads of from 50 to $60 \mathrm{lb} / \mathrm{ft}^{2}$ for orchestra floors of theaters and for floors of assembly halls with fixed seats, and $100 \mathrm{lb} / \mathrm{ft}^{2}$ for those with movable seats. Dance halls are assigned 100 to $120 \mathrm{lb} / \mathrm{ft}^{2}$. For schools, classrooms with fixed seats are required to be designed for 40 to $60 \mathrm{lb} / \mathrm{ft}^{2}$, and those with movable seats from 40 to $100 \mathrm{lb} / \mathrm{ft}^{2}$.

Information on loading of school floors appears in the report of the Department of Commerce Building Code Committee already referred to [3] 


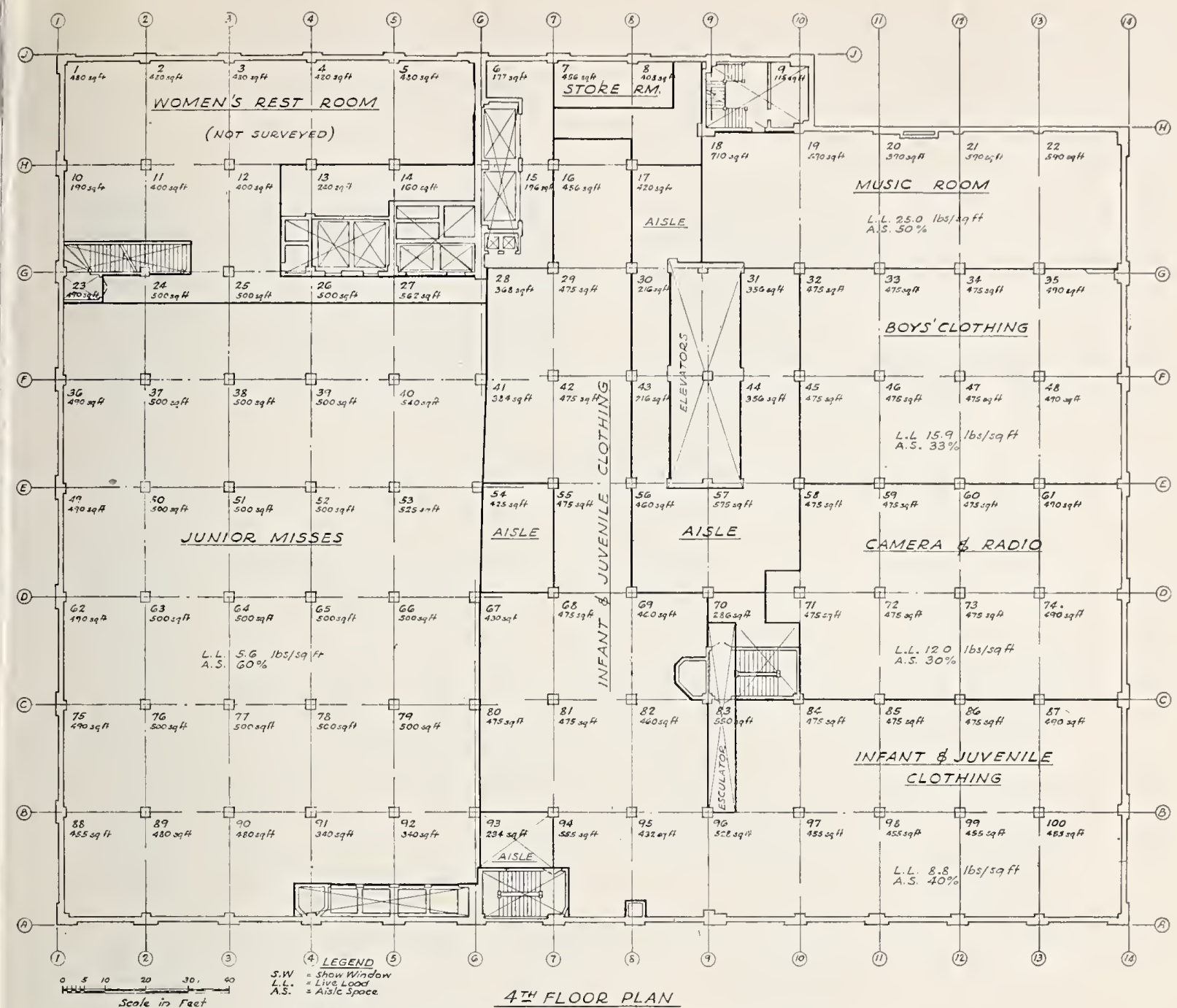

Ficure 2. Fourth-floor plan, department store, Washington, D. C.

Schools.-According to investigations by Norman M. Stineman (American Architect, April 11, 1923) a standard classroom has 736 square feet area and accommodates 45 pupils. The average weight of furniture and inmates is about 7,500 pounds, or 10 pounds per square foot. He estimates the maximum possible load as 2 adults in each seat and 30 around walls, giving total live load of 28 pounds per square foot.

In the course of loading tests for schoolhouse floors made by the Milwaukee Board of Education (Engineering News-Record, May 6, 1920) a room 24 feet 5 inches by 32 feet ( 781.3 square feet) normally for the accommodation of a teacher and 48 pupils was crowded with 258 pupils, filling all seats double, and all aisles and open spaces. There resulted a total weight per square foot, including desks, of 41.7 pounds.

Under normal conditions with 48 pupils at an average weight of 115.6 pounds, plus weight of desks and teachers, the average floor load was 10.83 pounds per square foot. Filled under normal conditions with adults, as in the case of night school, the load amounted to 12.9 pounds per square foot.

Other investigators put the live load in school classrooms normally filled at 14 pounds per square foot, and at 22 pounds if the aisles are crowded.
Crowded rooms.-Densely crowded gioups have been shown by several investigators to weigh at least 140 pounds per square foot, but those results were obtained by strenuous methods, and it is held unlikely that they will oecur under ordinary conditions. Observations of the loading obtained under normal conditions in the elevators at the Grand Central Terminal in New York showed a maximum of 73 persons on 92 square feet of floor area. With an estimated weight of 130 pounds each this gives a load of 100 pounds per square foot. Crowds of students at Iowa State University, packed for the purpose of testing baleony construction under dynamic loads weighed 116 pounds per square foot.

\section{Institutional Occupancy}

Such occupancies as hospitals, sanitariums, homes for the aged, and jails come under" "Institutional" classification. Building codes recommended by various organizations for national or regional use and building code standards agree on $40 \mathrm{lb} / \mathrm{ft}^{2}$ as a suitable figure for permissible mini- 
mum live load in private rooms. Figures for wards range from 40 to $80 \mathrm{lb} / \mathrm{ft}^{2}{ }^{2}$ Other figures are given for special locations.

The report of the Department of Commerce Building Code Committee [3] presents the following information:

Hospitals.-Through the courtesy of those in charge of the New York State hospitals for the insane at Brooklyn and Rochester, actual live-load measurements were obtained for several large wards in each institution. The data are in the following table:

A survey of a large dormitory in the Willard, N. Y., State hospital checks the above figures very closely. The room accommodated 86 beds in a total area of 2,600 square feet. The live loads were as follows:
Pounds

7,300

3,870

11,600

86 mattresses, etc., at 45 pounds_........

86 patients, at 135 pounds

22,770

Total load

8. 75

Average load per square foot

In 1940, inquiry was made of officials of the above institutions as to whether there had been any changed conditions since the original survey that might have caused changes in the live loads indicated. The information received was to the effect that nothing significant had occurred and that the live loads were probably about the same as those given in the 1924 report, from which the above is a quotation.

Live loads in crowded wards

\begin{tabular}{|c|c|c|c|c|c|}
\hline \multicolumn{6}{|c|}{ Brooklyn State Hospital } \\
\hline Ward number & Dimensions & $\begin{array}{c}\text { Total } \\
\text { number of } \\
\text { beds }\end{array}$ & $\begin{array}{l}\text { A verage } \\
\text { weight of } \\
\text { bed equip- } \\
\text { ment and } \\
\text { occupant }\end{array}$ & $\begin{array}{l}\text { Total floor } \\
\text { load }\end{array}$ & $\begin{array}{l}\text { Load per } \\
\text { square } \\
\text { foot }\end{array}$ \\
\hline 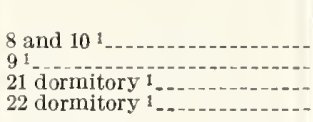 & 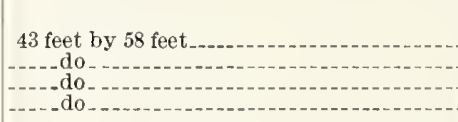 & $\begin{array}{l}62 \\
65 \\
62 \\
65\end{array}$ & $\begin{array}{l}\text { Pounds } \\
275 \\
275 \\
295 \\
295\end{array}$ & $\begin{array}{l}\text { Pounds } \\
17,050 \\
17,875 \\
18,290 \\
19,175\end{array}$ & $\begin{array}{l}\text { Pounds } \\
6.9 \\
7.2 \\
7.3 \\
7.7\end{array}$ \\
\hline \multicolumn{6}{|c|}{ Rochester State Insane Hospital } \\
\hline 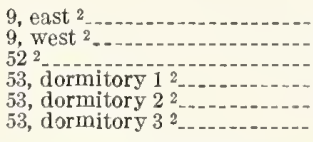 & $\begin{array}{l}49 \text { feet } 6 \text { inches by } 34 \text { feet } 6 \text { inches } \\
50 \text { feet by } 34 \text { feet } 6 \text { inches } 6 \\
48 \text { feet by } 28 \text { feet-at } \\
36 \text { feet by } 47 \text { feet } \\
21 \text { feet by } 36 \text { feet } \\
49 \text { feet by } 28 \text { feet. }\end{array}$ & $\begin{array}{l}46 \\
52 \\
36 \\
43 \\
18 \\
45\end{array}$ & $\begin{array}{l}256 \\
256 \\
276 \\
276 \\
276 \\
276\end{array}$ & $\begin{array}{r}11,776 \\
13,312 \\
9,936 \\
11,868 \\
4,968 \\
12,420\end{array}$ & $\begin{array}{l}6.9 \\
7.7 \\
7.5 \\
7.0 \\
6.6 \\
9.0\end{array}$ \\
\hline
\end{tabular}

Wards 8,9 , and 10, females, average weight, 145 pounds; domitories 21 and 22, males, average weight, 165 pounds. Radiators not included. Ward 9, females, average weight, 130 pounds; wards 52 and 53 , males, average weight, 150 pounds. Radiators not included.

\section{Industrial Occupancy}

Obviously, the "Industrial" classification will contain widely varying examples of floor loading, since it includes occupancies involved in manufacturing, fabrication, and assembly of all kinds of industrial products. Building codes recommended by various organizations for national or regional use and building code standards give minimum design loads from 75 to $125 \mathrm{lb} / \mathrm{ft}^{2}$ for light manufacturing. For heavy manufacturing, some give values of from 125 to $150 \mathrm{lb} / \mathrm{ft}^{2}$, and others do not assign any particular value.

Information has become available on several occupancies within this classification through the survey made by the Public Buildings Administration. The results are given below. Floor plans of each example surveyed are shown in figures 3 to 10 .

A number of different methods were employed in determining the loading in the examples given below. In some cases, all material found in the areas indicated was weighed; in others, typical items were weighed and the total weight computed.
Where neither of these methods seemed practicable, inquiry was made of the plant manager, manufacturer, or other source of information as to the weight of the item involved, or catalogs were consulted. In some instances, built-in items were measured and their weight calculated; this method was also used in the case of some movable items. Where weight of persons was included, this was based on the maximum number found to be present during normal operations and a unit weight of $150 \mathrm{lb}$ a person.

The particular methods used depended upon the conditions found. Special situations for which allowance had to be made are given in each case.

It will be apparent that the weights of machines and other heavy equipment have been averaged over fairly large areas. Although there is a tendency to place heavy machinery on ground floors or on special foundations reaching to the ground, some machines encountered in the survey were on upper floors and their weights shown in the tables were averaged over areas considerably greater than those immediately adjacent to the machines. Some instances of this kind will be cited in connection with buildings to which they apply. 


\section{Mattress Factory, Chicago, Ill.:}

Offiees were omitted from this survey. The results of the survey are given in tables 5 and 6. Figure 3 shows a floor in the faetory.

Table 5. Live loads in mattress factory, Chicago, Ill.

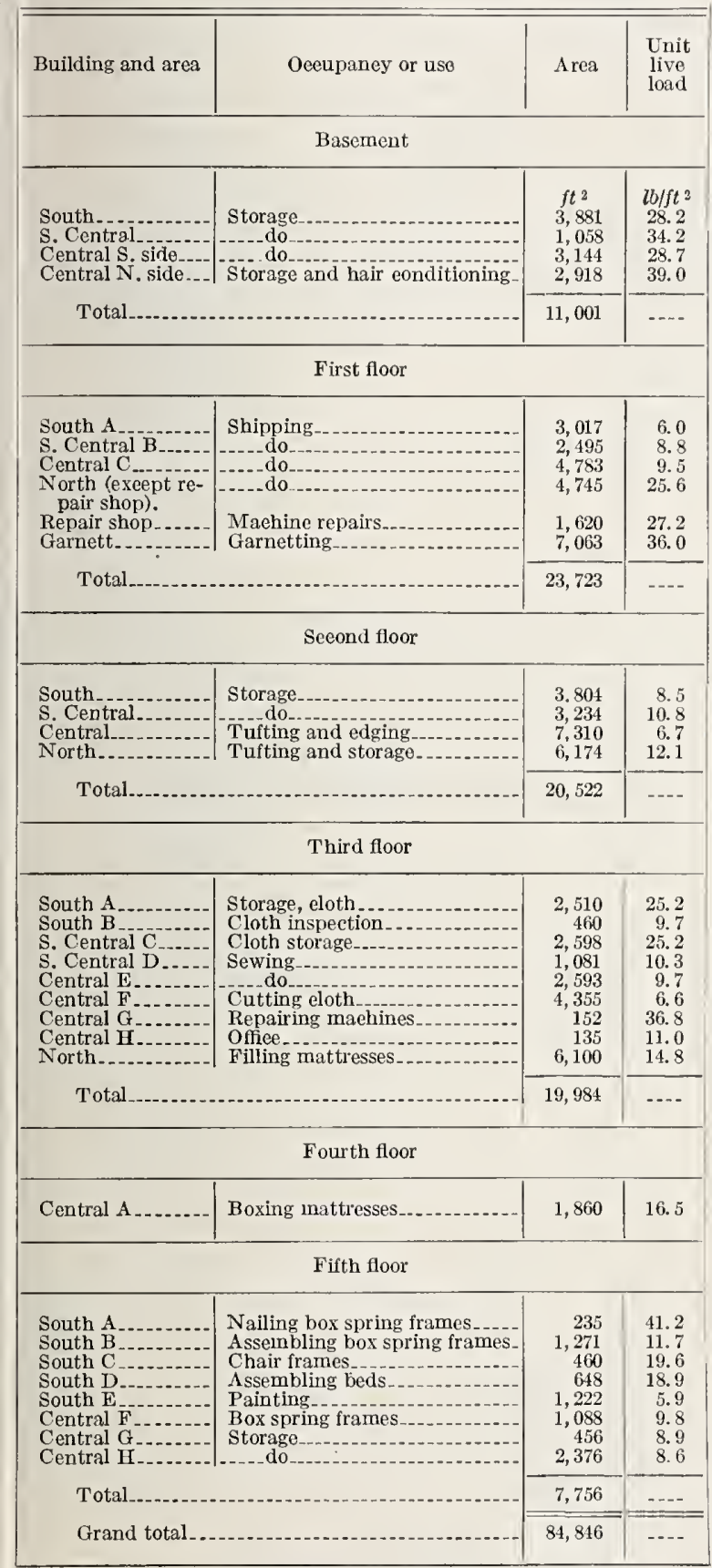

\section{Mattress Factory, Atlanta, Ga.:}

Only those buildings, or parts of buildings, in which some operation eoneerned with the making and shipping of felt and spring bed mattresses was being eonducted, were surveyed. Offiees were
TABLE 6. Variation in live lands in mattress factory, Chicago, Ill.

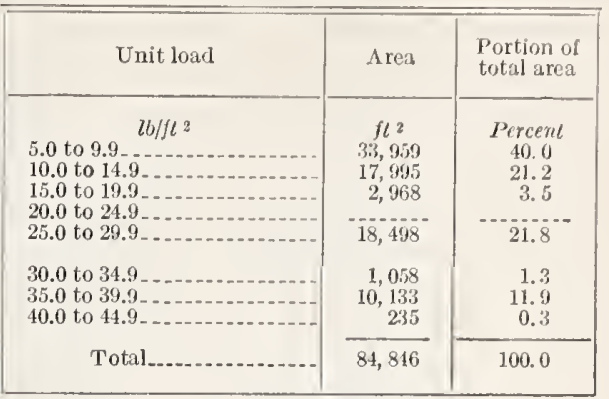

omitted. Temporary wood partitions were included as live load.

The heaviest maehines on a framed floor found in either of the mattress faetory surveys were garnetting maehines weighing about 25,500 lb apieee. The effeet of sueh eoneentrations may be illustrated by referenee to the second floor of building 3, whieh is shown on figure 4 . If the weight of one of these maehines is averaged over the two bays that it oceupies, the result is about $70 \mathrm{lb} / \mathrm{ft}^{2}$. On the other hand, if the weight is divided by the area of the base of the maehine alone, the average load is about $154 \mathrm{lb} / \mathrm{ft}^{2}$. The results of the survey are given in tables 7 and 8 . Figure 4 shows a floor in the faetory.

Taвle 7. Live loads in maltress factory, Allanta, Ga.

\begin{tabular}{|c|c|c|c|}
\hline $\begin{array}{c}\text { Panel or } \\
\text { area }\end{array}$ & Oecupaney or use & Area & $\begin{array}{l}\text { Unit live } \\
\text { load }\end{array}$ \\
\hline \multicolumn{4}{|c|}{ Building 1, Seeond floor } \\
\hline \multirow[t]{2}{*}{$\begin{array}{l}\mathrm{A} \\
\mathrm{B} \\
\mathrm{C} \\
\mathrm{D} \\
\mathrm{E}\end{array}$} & $\begin{array}{l}\text { Spring assembly } \\
\text { Spring storage } \\
\text { Spring assembly }\end{array}$ & $\begin{array}{r}f t^{2} \\
805 \\
900 \\
1,124 \\
453 \\
207\end{array}$ & $\begin{array}{r}l b / f \iota^{2} \\
12.6 \\
5.6 \\
5.8 \\
17.6 \\
15.5\end{array}$ \\
\hline & Total & 3,489 & ...... \\
\hline \multicolumn{4}{|c|}{ Building 2, Seeoud floor } \\
\hline $\begin{array}{l}\mathrm{A} \\
\mathrm{B} \\
\mathrm{C} \\
\mathrm{D} \\
\mathrm{E}\end{array}$ & $\begin{array}{l}\text { Temporary storage } \\
\text { Cotton felt mattress. } \\
\text { Mattress stapling } \\
\text { Mattress make-up } \\
\text { Matress tape edging }\end{array}$ & $\begin{array}{r}1,743 \\
1,312 \\
635 \\
635 \\
942\end{array}$ & $\begin{array}{l}8.0 \\
6.6 \\
6.2 \\
4.4 \\
7.9\end{array}$ \\
\hline $\begin{array}{c}\mathrm{F} \\
\mathrm{G} \\
\mathrm{H} \\
\mathrm{I} \\
\mathbf{J}\end{array}$ & $\begin{array}{l}\text { Mattress button tufting. } \\
\text { Mattress re-ginning } \\
\text { Spring receiving } \\
\text { Stapling } \\
\text { Cotton felt mattress. }\end{array}$ & $\begin{array}{r}1,243 \\
440 \\
472 \\
472 \\
864\end{array}$ & $\begin{array}{r}8.0 \\
24.0 \\
11.9 \\
8.0 \\
9.0\end{array}$ \\
\hline \multirow[t]{2}{*}{$\frac{\mathrm{K}}{\mathrm{L}}$} & $\begin{array}{l}\text { Cotton tufting } \\
\text { Roll edging }\end{array}$ & $\begin{array}{r}1,115 \\
950\end{array}$ & $\begin{array}{l}6.5 \\
7.5\end{array}$ \\
\hline & Total ... & 10,823 & - - - \\
\hline \multicolumn{4}{|c|}{ Building $2 \mathrm{~A}$, Seeond floor } \\
\hline $\begin{array}{l}\mathrm{A} \\
\mathrm{B} \\
\mathrm{C} \\
\mathrm{D} \\
\mathbf{E} \\
\mathrm{F} \\
\mathrm{G}\end{array}$ & $\begin{array}{l}\text { Box spring assembly } \\
\text { Box spring make-up } \\
\text { Box spring make-up } \\
\text { Box spring storage } \\
\text { Quilting tops.-. } \\
\text { Temporary storage } \\
\text { Paekaging. }\end{array}$ & $\begin{array}{r}\$ 16 \\
900 \\
648 \\
1,203 \\
1,450 \\
990 \\
2,115\end{array}$ & $\begin{array}{r}17.0 \\
11.0 \\
4.3 \\
5.2 \\
5.3 \\
17.4 \\
6.0\end{array}$ \\
\hline $\mathrm{G}$ & Total... & 8,152 & .....- \\
\hline
\end{tabular}




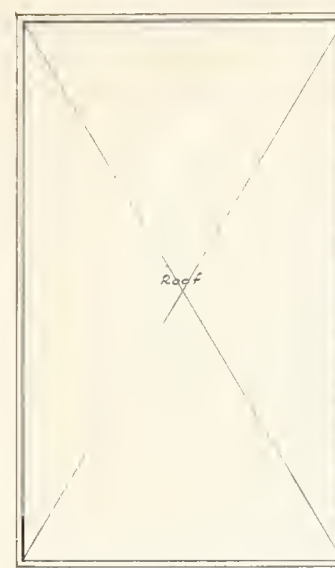

GARNETT BUILDING

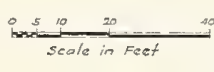

Figure 3. Second-floor plan, building 3, mattress factory, Chicago, Ill.

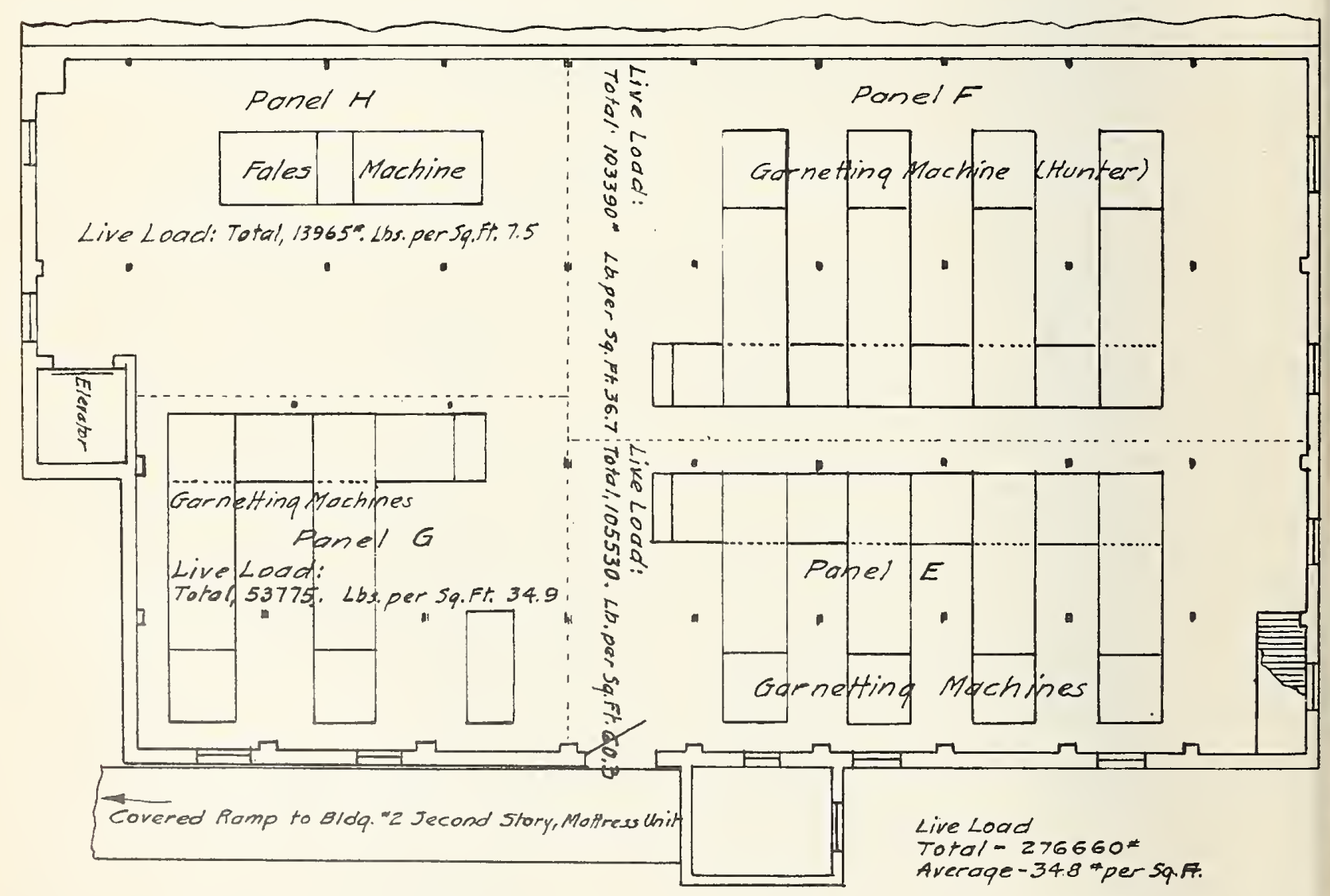

Scole: 1 Inch $=16$ Feet.

Garnetting unit.

Figure 4. Second-floor plan, building 3, mattress factory, Atlanta, Ga. 
TABLe 7. Live loads in mattress factory, Atlanta, Ga.-Con.

\begin{tabular}{|c|c|c|c|}
\hline $\begin{array}{l}\text { Panel or } \\
\text { area }\end{array}$ & Oeeupaney or use & Area & $\begin{array}{l}\text { Unit live } \\
\text { iond }\end{array}$ \\
\hline \multicolumn{4}{|c|}{ Building $2 \mathrm{~B}$, Second floor } \\
\hline A & $\begin{array}{l}\text { Stoek room } \\
\text { Sewing room - }\end{array}$ & $\begin{array}{l}\int t^{2} \\
1,556 \\
2,160\end{array}$ & $\begin{array}{c}l b / f t^{2} \\
59.3 \\
5.7\end{array}$ \\
\hline & Total_. & 3,716 & --.... \\
\hline
\end{tabular}

Building 3 , First floor

\begin{tabular}{|c|c|c|c|}
\hline $\begin{array}{l}\mathrm{A} \\
\mathrm{B} \\
\mathrm{C} \\
\mathrm{D}\end{array}$ & $\begin{array}{l}\text { Cotton eleaner, pieker } \\
\text { Cotton mixers } \\
\text { Cotton stores } \\
\text { Cotton batting, ete., stores }\end{array}$ & $\begin{array}{r}561 \\
1,030 \\
825 \\
6,170\end{array}$ & $\begin{array}{r}22.4 \\
6.5 \\
9.3 \\
15.2\end{array}$ \\
\hline & Total & 8,636 & -... \\
\hline
\end{tabular}

Building 3 , Seeond floor

\begin{tabular}{|c|c|c|c|}
\hline $\begin{array}{l}\mathrm{E} \\
\mathrm{F} \\
\mathrm{G} \\
\mathrm{H}\end{array}$ & $\begin{array}{l}\text { Garnetting } \\
\text { Garnetting } \\
\text { Garnetting } \\
\text { Fales }\end{array}$ & $\begin{array}{l}1,750 \\
2,816 \\
1,540 \\
1,853\end{array}$ & $\begin{array}{r}60.3 \\
36.7 \\
34.9 \\
7.5\end{array}$ \\
\hline & Total & 7,959 & ....... \\
\hline
\end{tabular}

Building 4, Garnett Annex

\begin{tabular}{|c|c|c|c|}
\hline All & Garnett Annex..... & 4,810 & 21.4 \\
\hline \multicolumn{4}{|c|}{ Building 5 , Cotton Warehouse } \\
\hline Whole & Cotton warehouse ... & 8,010 & 101.3 \\
\hline \multicolumn{4}{|c|}{ Building 6 , Shipping } \\
\hline All & Shipping........ & 15,640 & 27.6 \\
\hline \multicolumn{4}{|c|}{ Garnett Parts Stores } \\
\hline All & Garnett parts stores... & 740 & 93.9 \\
\hline \multicolumn{2}{|r|}{ Grand total } & 72,005 & .....- \\
\hline
\end{tabular}

TABLE 8. Variation in live loads in mattress factory, Atlanta, Ga.

\begin{tabular}{|c|c|c|}
\hline Unit load & Area & $\begin{array}{l}\text { Portion of } \\
\text { total area }\end{array}$ \\
\hline $\begin{array}{l}0 \mathrm{~b} / \mathrm{ft} 2 \\
0.0 \text { to } 4.9 \\
5.0 \text { to } 9.9 \\
10.0 \text { to } 14.9 \\
15.0 \text { to } 19.9 \\
20.0 \text { to } 24.9\end{array}$ & $\begin{array}{c}f t{ }^{2} \\
1,283 \\
22,046 \\
2,177 \\
8,626 \\
5,811\end{array}$ & $\begin{array}{c}\text { Percent } \\
1.8 \\
30.6 \\
3.0 \\
12.0 \\
8.1\end{array}$ \\
\hline $\begin{array}{l}25.0 \text { to } 29.9 \\
30.0 \text { to } 34.9 \\
35.0 \text { to } 39.9 \\
40.0 \text { to } 54.9\end{array}$ & $\begin{array}{r}15,640 \\
1,540 \\
2,816 \\
-1,556\end{array}$ & $\begin{array}{r}21.7 \\
2.1 \\
3.9 \\
2.2\end{array}$ \\
\hline $\begin{array}{l}60.0 \text { to } 64.9 \\
65.0 \text { to } 89.9 \\
90.0 \text { to } 94.9 \\
95.0 \text { to } 99.9 \\
100.0 \text { to } 104.9\end{array}$ & $\begin{array}{c}1,750 \\
-740 \\
-8,010\end{array}$ & $\begin{array}{c}2.5 \\
1.0 \\
11.1\end{array}$ \\
\hline Total & 72,005 & 100.0 \\
\hline
\end{tabular}

Men's Clothing Factory, New York, N. Y.:

The premises surveyed contain all operations from the receiving of the original bolt of cloth to the shipping of the finished suit or coat. 'The results of the survey are given in tables 9 and 10 . Figure 5 shows a floor in the factory.

TABLE 9. Live loads in men's clothing factory, New York, N. Y.

\begin{tabular}{|c|c|c|}
\hline Department & Area & $\begin{array}{l}\text { Unit } \\
\text { live load }\end{array}$ \\
\hline \multicolumn{3}{|c|}{ First Building, Firth floor } \\
\hline $\begin{array}{l}\text { Cutting } \\
\text { Shrinking and storage } \\
\text { Pattern design } \\
\text { Storage_- } \\
\text { Offiees }\end{array}$ & $\begin{array}{r}f t^{2} \\
16,285 \\
4,275 \\
440 \\
7,085 \\
3,145\end{array}$ & $\begin{array}{r}(b) / f t^{2} \\
11.0 \\
13.3 \\
8.6 \\
19.6 \\
10.8\end{array}$ \\
\hline Total & 31,230 & ...... \\
\hline \multicolumn{3}{|c|}{ First Building, Sixth floor } \\
\hline $\begin{array}{l}\text { Reeeiving and storage } \\
\text { Suit storage } \\
\text { Labels and assembly } \\
\text { Paeking } \\
\text { Corridor }\end{array}$ & $\begin{array}{l}5,005 \\
6,800 \\
1,680 \\
2,980 \\
460\end{array}$ & $\begin{array}{r}8.7 \\
11.1 \\
13.6 \\
13.5 \\
10.5\end{array}$ \\
\hline Total & 16,925 & -..... \\
\hline \multicolumn{3}{|c|}{ Seeond Building, Third floor } \\
\hline $\begin{array}{l}\text { Offiee. } \\
\text { Coat room and storage } \\
\text { Storage (cloth in hundles) } \\
\text { Maehine repair } \\
\text { Hand sewing No. } 1\end{array}$ & $\begin{array}{r}190 \\
465 \\
315 \\
115 \\
1,350\end{array}$ & $\begin{array}{r}11.1 \\
15.1 \\
27.9 \\
47.0 \\
6.9\end{array}$ \\
\hline $\begin{array}{l}\text { Hand sewing No. } 2 \\
\text { Hand sewing No. } 3 \\
\text { Storage and reeeiving } \\
\text { Pressing No. } 1 \text {. } \\
\text { Pressing No. } 2\end{array}$ & $\begin{array}{r}5,135 \\
1,215 \\
1,400 \\
840 \\
4,445\end{array}$ & $\begin{array}{r}7.9 \\
8.0 \\
8.8 \\
20.3 \\
19.9\end{array}$ \\
\hline $\begin{array}{l}\text { Maehine sewing No. 1. } \\
\text { Maehine sewing No. } 2\end{array}$ & $\begin{array}{l}1,475 \\
1,860\end{array}$ & $\begin{array}{l}16.0 \\
17.3\end{array}$ \\
\hline Total & 18,805 & 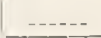 \\
\hline Grand total & 66,960 & -..... \\
\hline
\end{tabular}

TABLE 10. Variation in live loads in men's clothing factory, New York, N. Y.

\begin{tabular}{|c|c|c|}
\hline Unit load & Area & $\begin{array}{l}\text { Portion of } \\
\text { total area }\end{array}$ \\
\hline $\begin{array}{r}5.0 \text { to } 9.9 \mathrm{~h} / \mathrm{ft} 2 \\
10.0 \text { to } 14.9 \\
15.0 \text { to } 19.9 \\
20.0 \text { to } 24.9 \\
25.0 \text { to } 29.9 \\
30.0 \text { to } 44.9 \\
45.0 \text { to } 49.5\end{array}$ & $\begin{array}{r}f t^{2} \\
14,545 \\
35,815 \\
15,330 \\
840 \\
315 \\
115\end{array}$ & $\begin{array}{l}\text { Percent } \\
21.7 \\
53.5 \\
22.9 \\
1.3 \\
0.4 \\
.2\end{array}$ \\
\hline Total & 66,960 & 100.0 \\
\hline
\end{tabular}




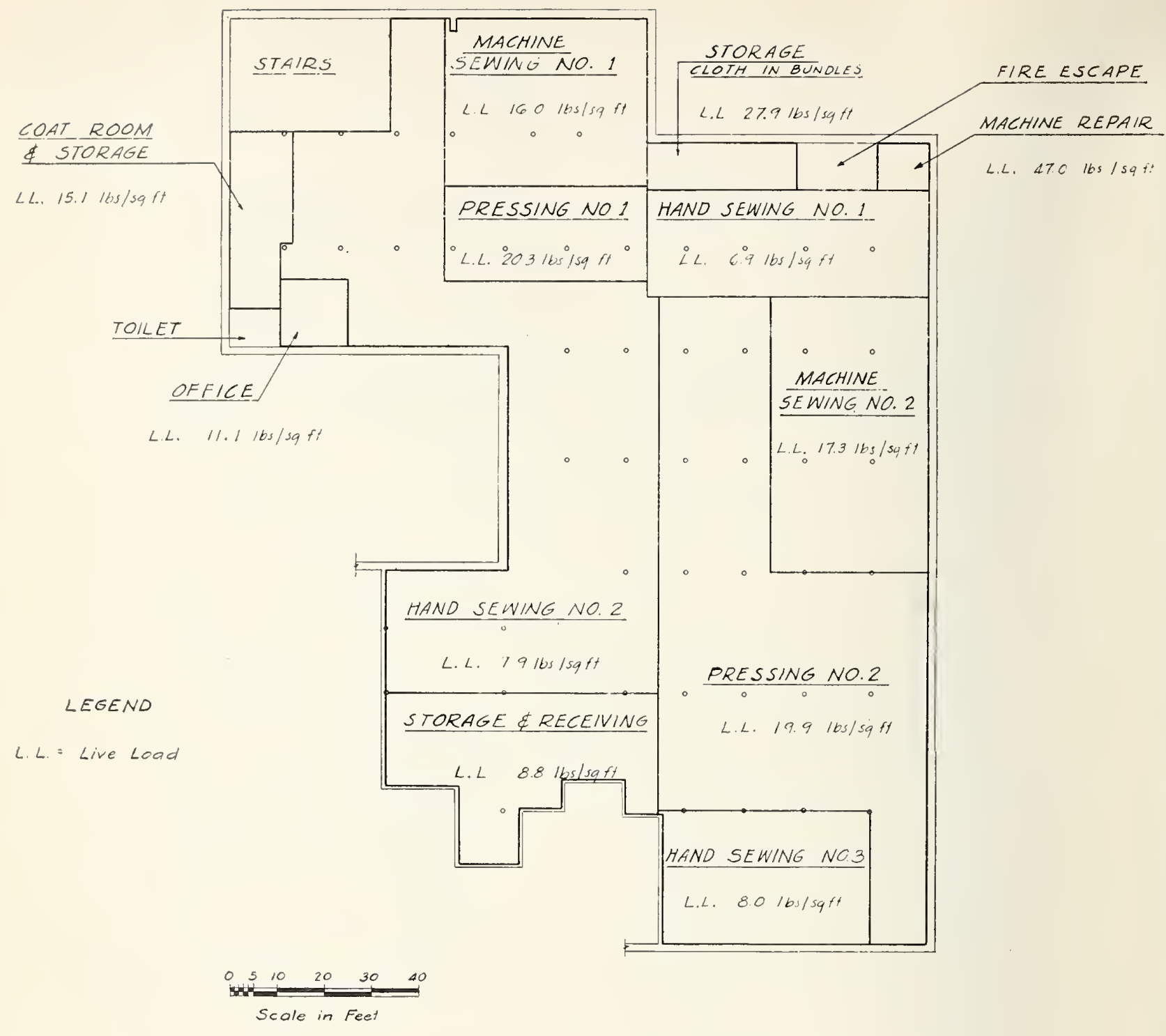

$\frac{\text { THIRD }}{\text { (SECOND BUILDING) }}$

Figure 5. Third-floor plan, men's clothing factory, New York, N. Y. 
Dress Factory, Philadelphia, Pa.:

No aisle space was considered. The counts of persons taken are belioved to represent adequatcly the maximum live load caused by people under similar factory conditions. The results of the survey are given in tables 11 and 12 . Figure 6 shows a floor in the factory.

Table 11. Live loads in dress factory, Philadelphia, Pa.

\begin{tabular}{|c|c|c|}
\hline Department & Area & $\begin{array}{l}\text { Unit live } \\
\text { load }\end{array}$ \\
\hline \multicolumn{3}{|l|}{ Second floor } \\
\hline $\begin{array}{l}\text { Cloth storage } \\
\text { Buttons, thread, etc. (storage) } \\
\text { Sample dresses (storage) } \\
\text { Stationery storage } \\
\text { Storage and shipping }\end{array}$ & $\begin{array}{l}f t{ }^{2} \\
850 \\
515 \\
160 \\
125 \\
5,420\end{array}$ & $\begin{array}{r}l b / \int t^{2} \\
38.9 \\
24.2 \\
9.4 \\
41.5 \\
9.5\end{array}$ \\
\hline $\begin{array}{l}\text { Women's dressing room } \\
\text { Designers' office- } \\
\text { Financial office- } \\
\text { Front office. } \\
\text { Anteroom }\end{array}$ & $\begin{array}{r}280 \\
1.650 \\
1,060 \\
1,085 \\
75\end{array}$ & $\begin{array}{r}7.7 \\
9.0 \\
11.1 \\
14.0 \\
15.4\end{array}$ \\
\hline $\begin{array}{l}\text { Men's room } \\
\text { Closet-1. } \\
\text { Hall }\end{array}$ & $\begin{array}{r}115 \\
60 \\
895\end{array}$ & $\begin{array}{r}21.4 \\
60.5 \\
8.4\end{array}$ \\
\hline Total & 12,290 & $-\ldots$ \\
\hline \multicolumn{3}{|l|}{ Third floor } \\
\hline $\begin{array}{l}\text { Emergency restroom } \\
\text { Office } \\
\text { Pattern storage } \\
\text { Restroom and reserve area. } \\
\text { Sewing room }\end{array}$ & $\begin{array}{r}145 \\
195 \\
80 \\
2,410 \\
2,635\end{array}$ & $\begin{array}{r}9.0 \\
15.1 \\
34.4 \\
5.8 \\
13.8\end{array}$ \\
\hline 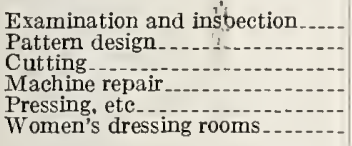 & $\begin{array}{r}490 \\
700 \\
2,415 \\
250 \\
2,915 \\
215\end{array}$ & $\begin{array}{r}19.4 \\
6.7 \\
7.1 \\
36.7 \\
5.5 \\
15.2\end{array}$ \\
\hline Total & 12,450 & - \\
\hline Grand total ... & 24,740 & - \\
\hline
\end{tabular}

TABLE 12. Variation in live loads in dress factory, Philadelphia, Pa.

\begin{tabular}{|c|c|c|}
\hline Unit load & Area & $\begin{array}{l}\text { Portion of } \\
\text { total area }\end{array}$ \\
\hline $\begin{array}{l}5 \mathrm{l} / \mathrm{ft} 2 \\
5.0 \text { to } 9.9 \\
10.0 \text { to } 14.9 \\
15.0 \text { to } 19.9 \\
20.0 \text { to } 24.9 \\
25.0 \text { to } 29.9\end{array}$ & $\begin{array}{r}f t^{2} \\
16,990 \\
4,780 \\
975 \\
630 \\
\end{array}$ & $\begin{array}{l}\text { Percent } \\
68.8 \\
19.4 \\
3.9 \\
2.5 \\
-\end{array}$ \\
\hline $\begin{array}{l}30.0 \text { to } 34.9 \\
35.0 \text { to } 39.9 \\
40.0 \text { to } 44.5 \\
60.0 \text { to } 59.9\end{array}$ & $\begin{array}{r}80 \\
1,100 \\
125 \\
-60\end{array}$ & $\begin{array}{c}0.3 \\
4.4 \\
0.5 \\
-0.2\end{array}$ \\
\hline Total.. & 24,740 & 100.0 \\
\hline
\end{tabular}

Furniture Factory, Getlysburg, Pa.:

The results of the survey are given in tables 13 and 14. Figure 7 shows the plan of the factory.

TABLE 13. Live loads in furniture factory, reltysthurg, P'a.

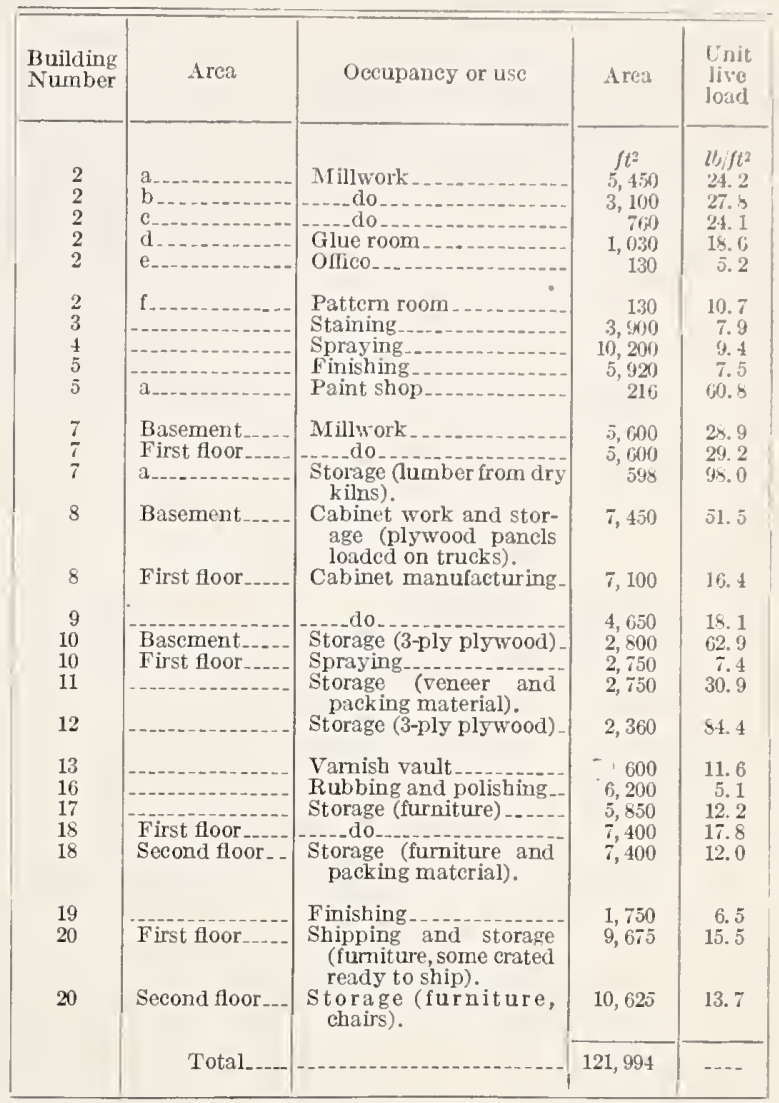

TABLE 14. Variation in live loads in furniture factory, Gettysburg, Pa.

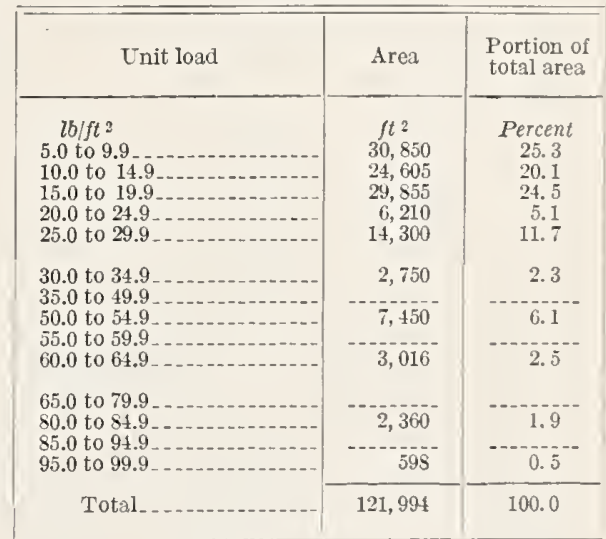




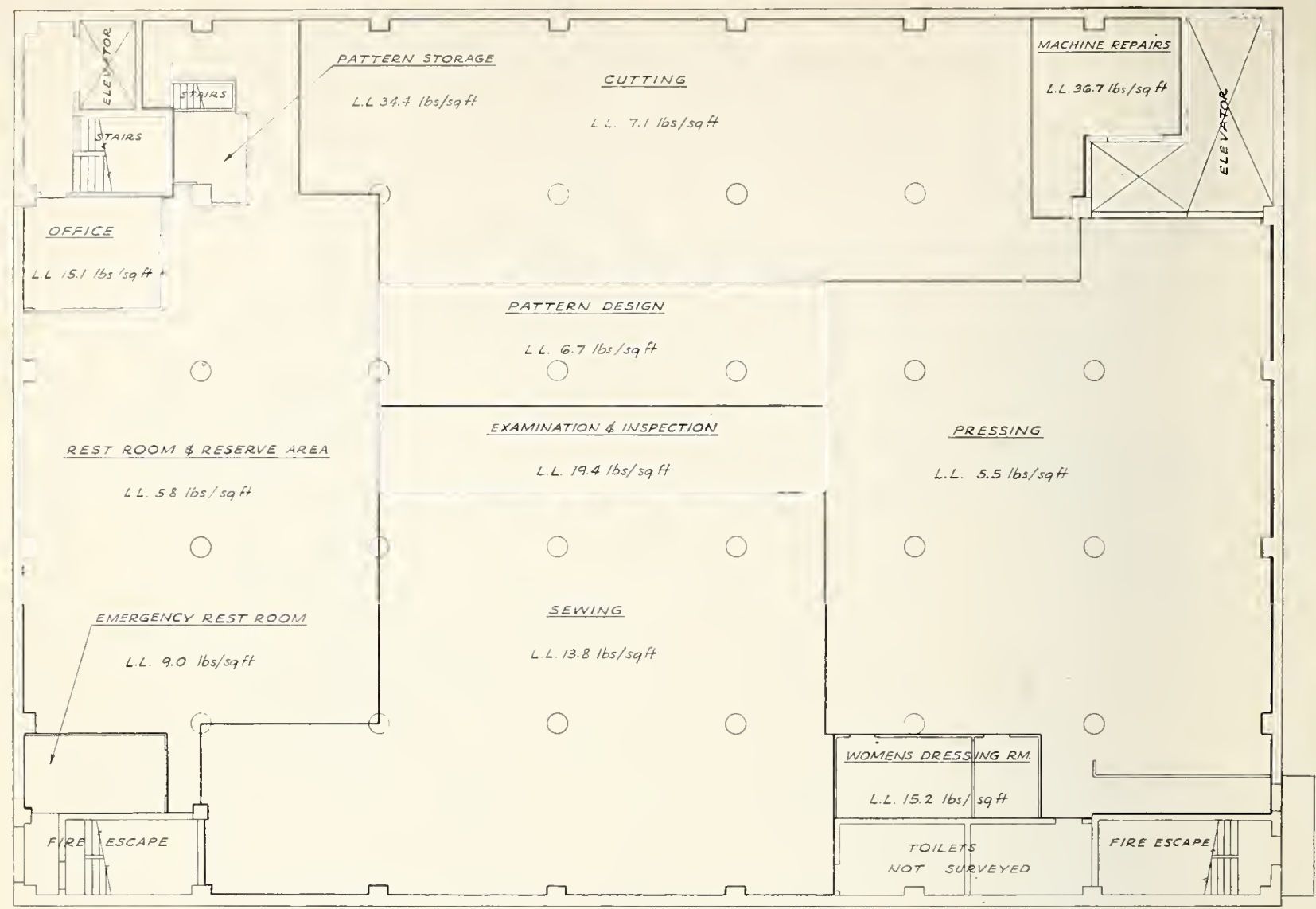

LEGEND

3RD FLOOR PLAN

L.L. = Live Load

Figure 6. Third-floor plan, dress factory, Philadelphia, Pa.

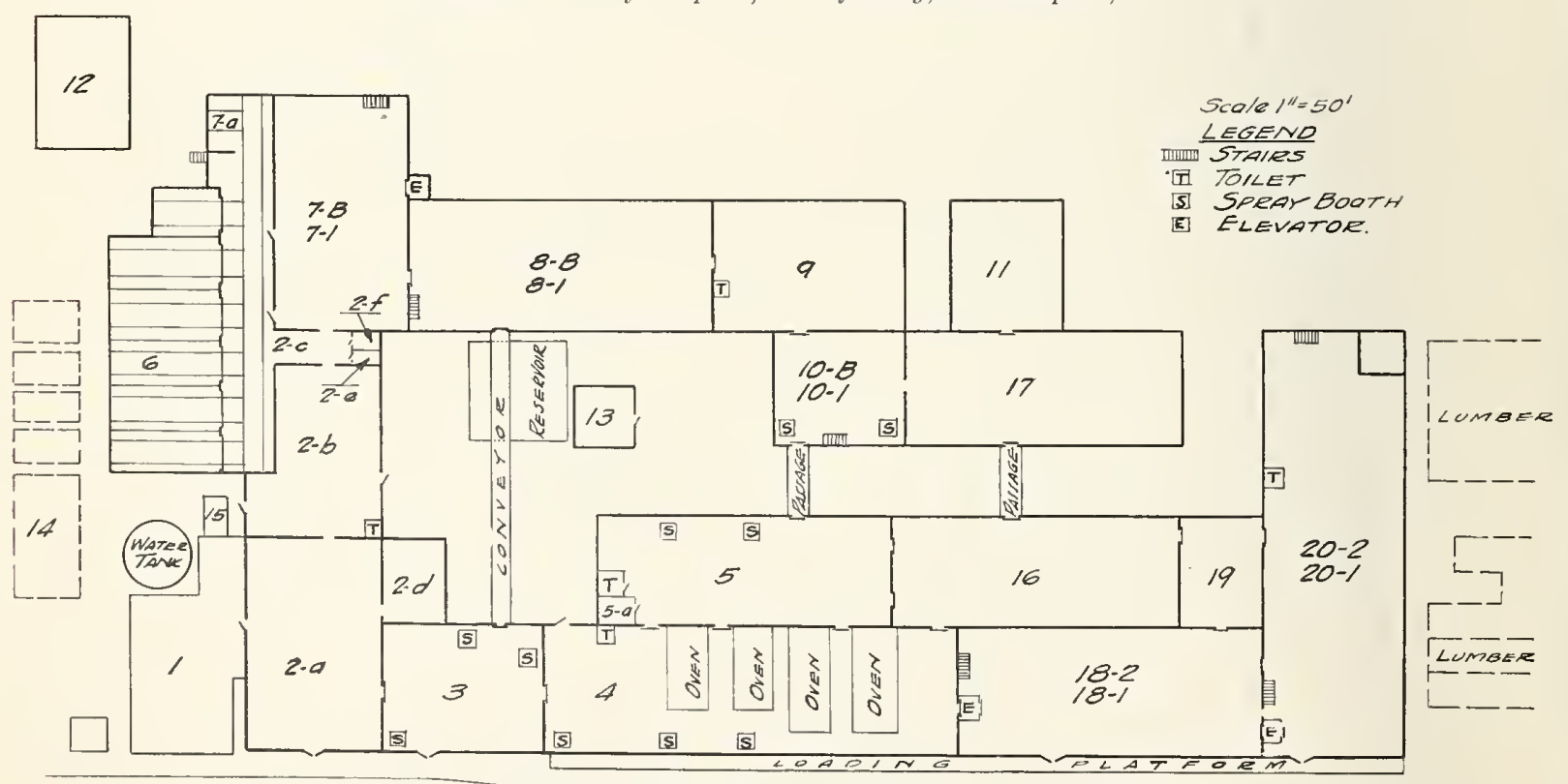

Figtre 7. Furniture factory, Gettysburg, Pa. 

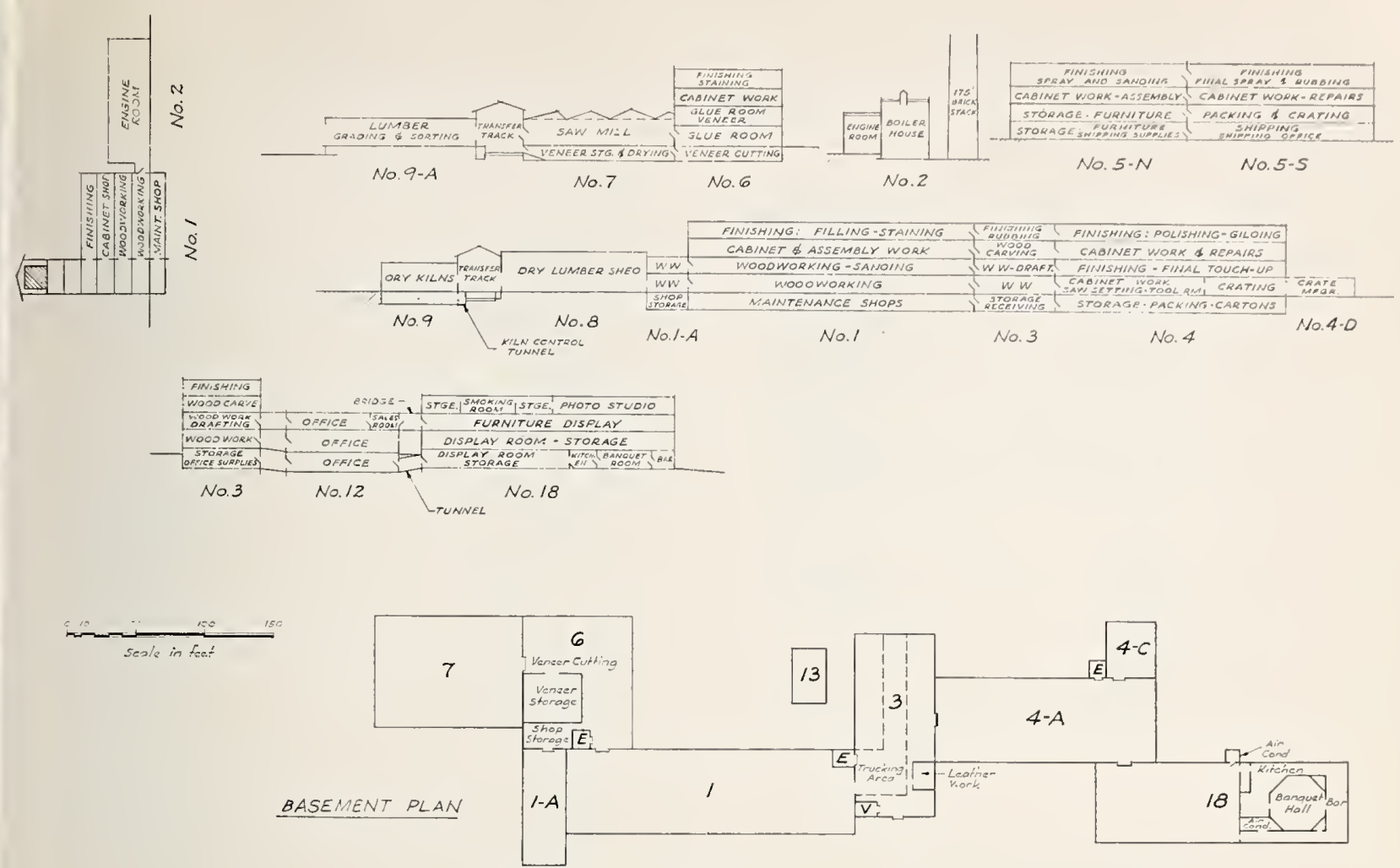

Figure 8. Furniture factory, Grand Rapids, Mich.

Furniture Factory, Grand Rapids, Mich.:

This furniture plant produces, as a specialty, a wide variety of utilitarian and decorative tables that are of high quality in construction and design. All of the buildings, or portions of buildings, occupied in the actual production, shipping, and display of furniture were surveyed. Office space and service buildings were not included in the survey. The heaviest of the machines found on framed floors in this survey were an automatic shaper, weighing $12,000 \mathrm{lb}$, on the first floor of building 3 , a hydraulic press, weighing $15,000 \mathrm{lb}$, on the second floor of building 6 , and a joiner, gluer, and drier, weighing $12,000 \mathrm{lb}$, on the first floor of building 7 . The areas occupied by these machines were not available. The results of the survey are given in tables 15 and 16 . Figure 8 shows the basement plan of the factory and the operations on the several floors.

TABLE 15. Live loads in furniture factory, Grand Rapids, Mich.

\begin{tabular}{|c|c|c|c|c|}
\hline $\begin{array}{l}\text { Building } \\
\text { number }\end{array}$ & Floor & Occupancy or use & Area & $\begin{array}{l}\text { Unit } \\
\text { live } \\
\text { load }\end{array}$ \\
\hline & & & $f t^{2}$ & $l b / f t^{2}$ \\
\hline $\begin{array}{l}1 \\
1\end{array}$ & $\begin{array}{c}\mathrm{B} \\
1\end{array}$ & $\begin{array}{l}\text { Maintenance shops } \\
\text { Woodworking }\end{array}$ & $\begin{array}{l}11,500 \\
11,500\end{array}$ & $\begin{array}{l}21.9 \\
20.5\end{array}$ \\
\hline 1 & 2 & do & 11,500 & 15.2 \\
\hline 1 & 3 & Cabinet and assembly work & 11,500 & 5.4 \\
\hline 1 & 4 & Finishing $\ldots$ & 11,500 & 4.1 \\
\hline
\end{tabular}

TABLE 15. Live loads in furniture factory, Grand Rapids, Mich.--Continued

\begin{tabular}{|c|c|c|c|c|}
\hline $\begin{array}{l}\text { Building } \\
\text { number }\end{array}$ & Floor & Oceupancy or use & Area & $\begin{array}{l}\text { Unit } \\
\text { live } \\
\text { load }\end{array}$ \\
\hline $\begin{array}{c}1-\mathrm{A} \\
1-\mathrm{A} \\
1-\mathrm{A} \\
3\end{array}$ & $\begin{array}{c}\mathrm{B} \\
1 \\
2 \\
\mathrm{~B} \\
-\end{array}$ & $\begin{array}{l}\text { Storage } \\
\text { Woodworking } \\
\text { do } \\
\text { Storage. } \\
\text { Leather room }\end{array}$ & $\begin{array}{l}f t^{2} \\
2,900 \\
2,900 \\
2,900 \\
5,730 \\
500\end{array}$ & $\begin{array}{c}l b / f t^{2} \\
23.1 \\
8.7 \\
30.0 \\
27.9 \\
27.6\end{array}$ \\
\hline $\begin{array}{c}3 \\
3 \\
-3 \\
3\end{array}$ & $\begin{array}{l}1 \\
2 \\
2 \\
3 \\
4\end{array}$ & $\begin{array}{l}\text { Woodworking } \\
\text { Woodworking and drafting } \\
\text { Drafting } \\
\text { Cabinet shop wood carring } \\
\text { Finishing-rubbing }\end{array}$ & $\begin{array}{r}8,800 \\
7,990 \\
860 \\
9,780 \\
8,850\end{array}$ & $\begin{array}{r}22.5 \\
11.1 \\
13.2 \\
16.4 \\
3.6\end{array}$ \\
\hline $\begin{array}{l}4-\mathrm{A} \\
4-\mathrm{C} \\
4-\mathrm{A} \\
4-\mathrm{A} \\
4-\mathrm{B}\end{array}$ & $\begin{array}{l}B \\
B \\
1 \\
1 \\
1\end{array}$ & $\begin{array}{l}\text { Storage. } \\
\text { do } \\
\text { Cabinet shop } \\
\text { Saw setting and tool shop... } \\
\text { Crating }\end{array}$ & $\begin{array}{l}9,250 \\
1.200 \\
+.960 \\
\quad 600 \\
3,600\end{array}$ & $\begin{array}{r}30.9 \\
24.1 \\
11.6 \\
23.7 \\
9.3\end{array}$ \\
\hline $\begin{array}{l}4-\mathrm{C} \\
4-\mathrm{D} \\
4-\mathrm{E} \\
4 \\
4\end{array}$ & $\begin{array}{l}1 \\
1 \\
1 \\
2 \\
3\end{array}$ & $\begin{array}{l}\text { Shipment make-up } \\
\text { Crate manufacture } \\
\text { Cabinet shop } \\
\text { Finishing-final } \\
\text { Cabinet work and repair shop }\end{array}$ & $\begin{array}{r}1,200 \\
4,990 \\
3,750 \\
10,932 \\
10,750\end{array}$ & $\begin{array}{r}6.7 \\
14.9 \\
7.3 \\
6.3 \\
4.6\end{array}$ \\
\hline $\begin{array}{l}4 \\
5-\mathrm{N} \\
5-\mathrm{S} \\
5-\mathrm{S} \\
5-\mathrm{N}\end{array}$ & $\begin{array}{l}4 \\
1 \\
1 \\
1 \\
2\end{array}$ & $\begin{array}{l}\text { Finishing-rubbing, gilding } \\
\text { Storage.- } \\
\text { Shipping- } \\
\text { Shipping office } \\
\text { Storage }\end{array}$ & $\begin{array}{r}10,732 \\
15,409 \\
15,000 \\
204 \\
15,200\end{array}$ & $\begin{array}{r}\text { 4. } 4 \\
23.8 \\
3.4 \\
6.7 \\
\text { T. } 0\end{array}$ \\
\hline $\begin{array}{l}5-\mathrm{S} \\
5-\mathrm{N} \\
5-\mathrm{S} \\
5-\mathrm{N} \\
5-\mathrm{S}\end{array}$ & $\begin{array}{l}2 \\
3 \\
3 \\
4 \\
4\end{array}$ & $\begin{array}{l}\text { Packing and erating } \\
\text { Cabinet work, assembly } \\
\text { Cabinet work, repairs } \\
\text { Finishing }\end{array}$ & $\begin{array}{l}15,200 \\
15,300 \\
15,300 \\
15,300 \\
15,300\end{array}$ & $\begin{array}{l}4.4 \\
4.3 \\
2.3 \\
2.3 \\
3.1\end{array}$ \\
\hline
\end{tabular}


TABLE 15. Live loads in furniture factory, Grand Rapids, Mich-Continued

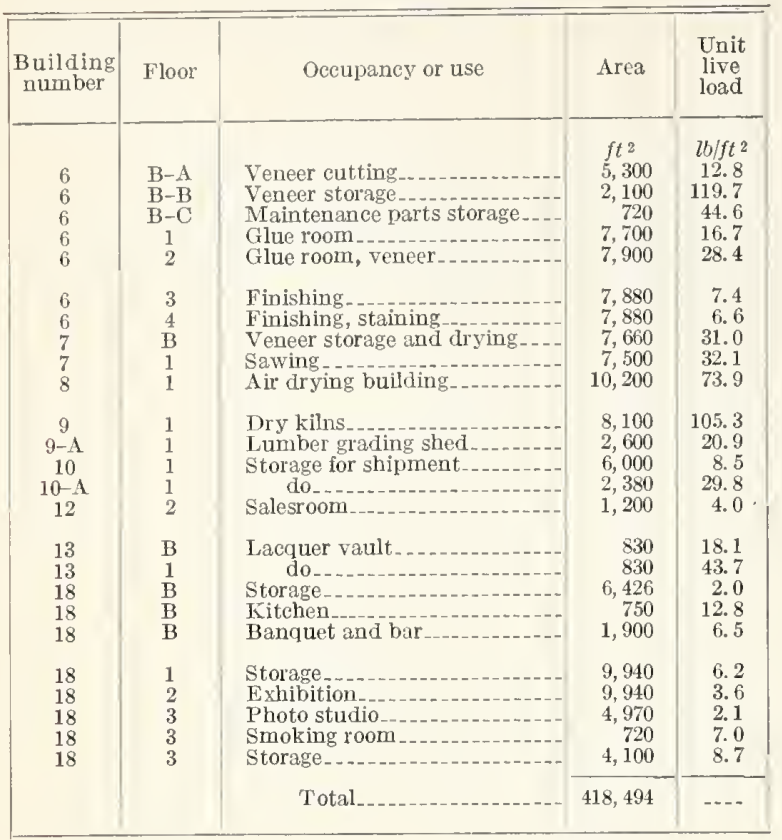

TABLE 16. Variation in live loads in furniture factory, Grand Rapids, Mich.

\begin{tabular}{|c|c|c|}
\hline Unit load & Area & $\begin{array}{l}\text { Portion } \\
\text { of total } \\
\text { area }\end{array}$ \\
\hline $\begin{array}{l}\mathrm{lb} / \mathrm{ft} 2 \\
0.0 \text { to } 4.9 \\
5.0 \text { to } 9.9 \\
10.0 \text { to } 14.9 \\
15.0 \text { to } 19.9 \\
20.0 \text { to } 24.9\end{array}$ & $\begin{array}{rl}f t & 2 \\
155,828 \\
87,706 \\
24,850 \\
29,810 \\
54,500\end{array}$ & $\begin{array}{c}\text { Percent } \\
37.2 \\
21.0 \\
6.0 \\
7.1 \\
13.0\end{array}$ \\
\hline $\begin{array}{l}25.0 \text { to } 29.9 \\
30.0 \text { to } 34.9 \\
35.0 \text { to } 39.9 \\
40.0 \text { to } 44.9 \\
45.0 \text { to } 69.9\end{array}$ & $\begin{array}{r}16,510 \\
27,340 \\
-1,550 \\
-\end{array}$ & $\begin{array}{c}4.0 \\
6.5 \\
0.4 \\
0.4\end{array}$ \\
\hline $\begin{array}{l}70.0 \text { to } 74.9 \\
75.0 \text { to } 104.9 \\
105.0 \text { to } 109.9 \\
110.0 \text { to } 114.9 \\
115.0 \text { to } 119.9\end{array}$ & $\begin{array}{r}10,200 \\
8,100 \\
2,100\end{array}$ & $\begin{array}{c}2.4 \\
1.9 \\
0.5\end{array}$ \\
\hline Total & 418,494 & 100.0 \\
\hline
\end{tabular}

Newspaper Plant, Washington, D. C.:

This survey included the building housing the printing plant and some offices of the newspaper. The heaviest machines were the presses, which were located on the first floor; however, these machines extended through the first floor and were supported on special pile footings, consequently were not included in the survey. The results of the survey are given in tables 17 and 18 . Figure 9 shows a floor in the plant.
TABLE 17. Live loads in newspaper plant, Washington, D. C.

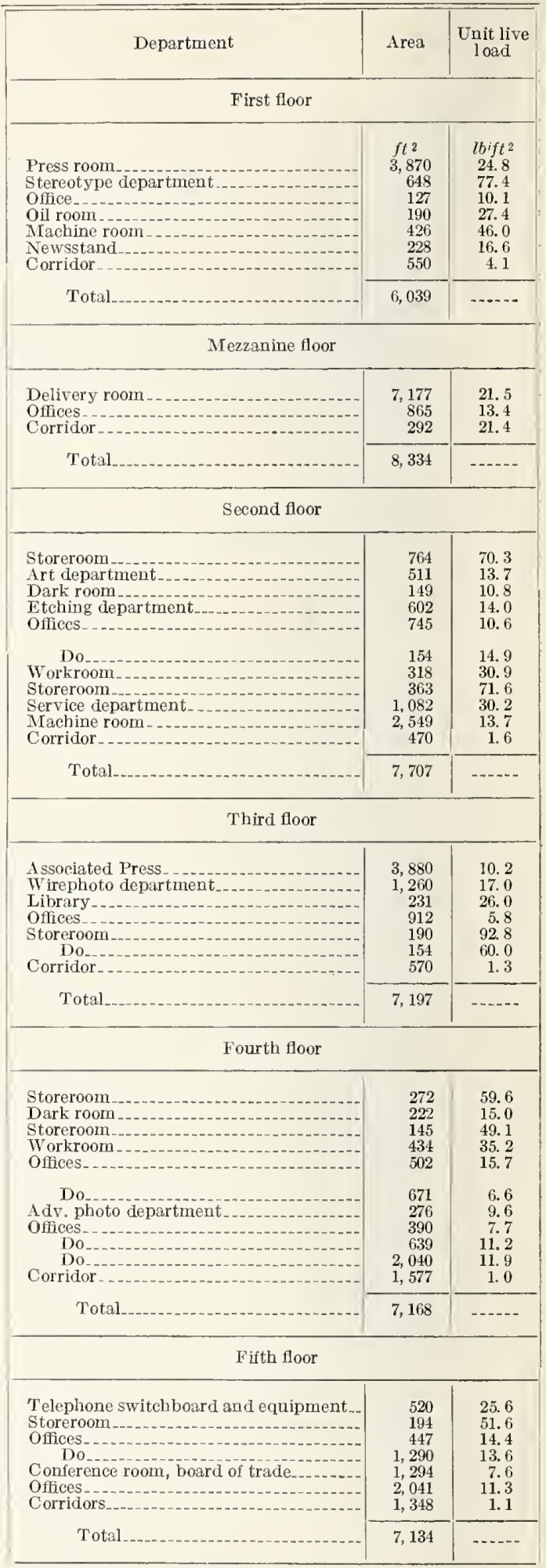


TABLE 17. Live loads in newspaper plant, Washington, D. C.-Continued

\begin{tabular}{|c|c|c|}
\hline Department & Area & $\begin{array}{l}\text { Unit live } \\
\text { load }\end{array}$ \\
\hline \multicolumn{3}{|l|}{ Sixth floor } \\
\hline $\begin{array}{l}\text { Offees } \\
\text { Corridor } \\
\text { Lobby } \\
\text { Offiee } \\
\text { Advertising and aceounting }\end{array}$ & $\begin{array}{r}f t^{2} \\
1,786 \\
740 \\
371 \\
324 \\
3,890\end{array}$ & $\begin{array}{r}l b / f t^{2} \\
9.7 \\
6.3 \\
2.0 \\
16.5 \\
21.0\end{array}$ \\
\hline Total_.. & 7,111 & ....... \\
\hline \multicolumn{3}{|l|}{ Seventh floor } \\
\hline $\begin{array}{l}\text { Offee } \\
\text { Do } \\
\text { Art oorm } \\
\text { Photographers } \\
\text { De ntallaboratory } \\
\text { City room } \\
\text { Corridor } \\
\text { Lobby. }\end{array}$ & $\begin{array}{r}1,900 \\
140 \\
496 \\
439 \\
126 \\
3,446 \\
692 \\
371\end{array}$ & $\begin{array}{r}11.4 \\
32.5 \\
18.6 \\
9.9 \\
17.5 \\
15.2 \\
3.2 \\
2.0\end{array}$ \\
\hline Total & 7,610 & - \\
\hline
\end{tabular}

TABLE 17. Live loads in newspaper plant, Washington, D. C.-Continued

\begin{tabular}{|c|c|c|}
\hline Department & Area & $\begin{array}{l}\text { Enit live } \\
\text { load }\end{array}$ \\
\hline \multicolumn{3}{|l|}{ Sighth floor } \\
\hline $\begin{array}{l}\text { Sureotype room } \\
\text { Cumposing room } \\
\text { Othee. } \\
\text { Lobby }\end{array}$ & $\begin{array}{r}f t^{2} \\
1,296 \\
6,300 \\
54 \\
176\end{array}$ & $\begin{array}{c}\text { W/ft }{ }^{2} \\
73.2 \\
58.2 \\
13.1 \\
4.3\end{array}$ \\
\hline Total & 7,826 & ....... \\
\hline \multicolumn{3}{|l|}{ Ninth floor } \\
\hline $\begin{array}{l}\text { Pieture file room } \\
\text { Monotype room } \\
\text { Uomposing room A } \\
\text { Uomposing room B } \\
\text { Uffiee. }\end{array}$ & $\begin{array}{r}914 \\
390 \\
1,600 \\
990 \\
232\end{array}$ & $\begin{array}{l}26.3 \\
57.2 \\
54.4 \\
14.2 \\
13.9\end{array}$ \\
\hline $\begin{array}{l}\text { Storeroom } \\
\text { Offiee } \\
\text { Lobby } \\
\text { Loeker room }\end{array}$ & $\begin{array}{r}165 \\
54 \\
300 \\
1,494\end{array}$ & $\begin{array}{r}66.0 \\
6.2 \\
6.5 \\
13.8\end{array}$ \\
\hline Total & 6,139 & $\ldots . . .$. \\
\hline Grand total & 72,265 & $\ldots$ \\
\hline
\end{tabular}

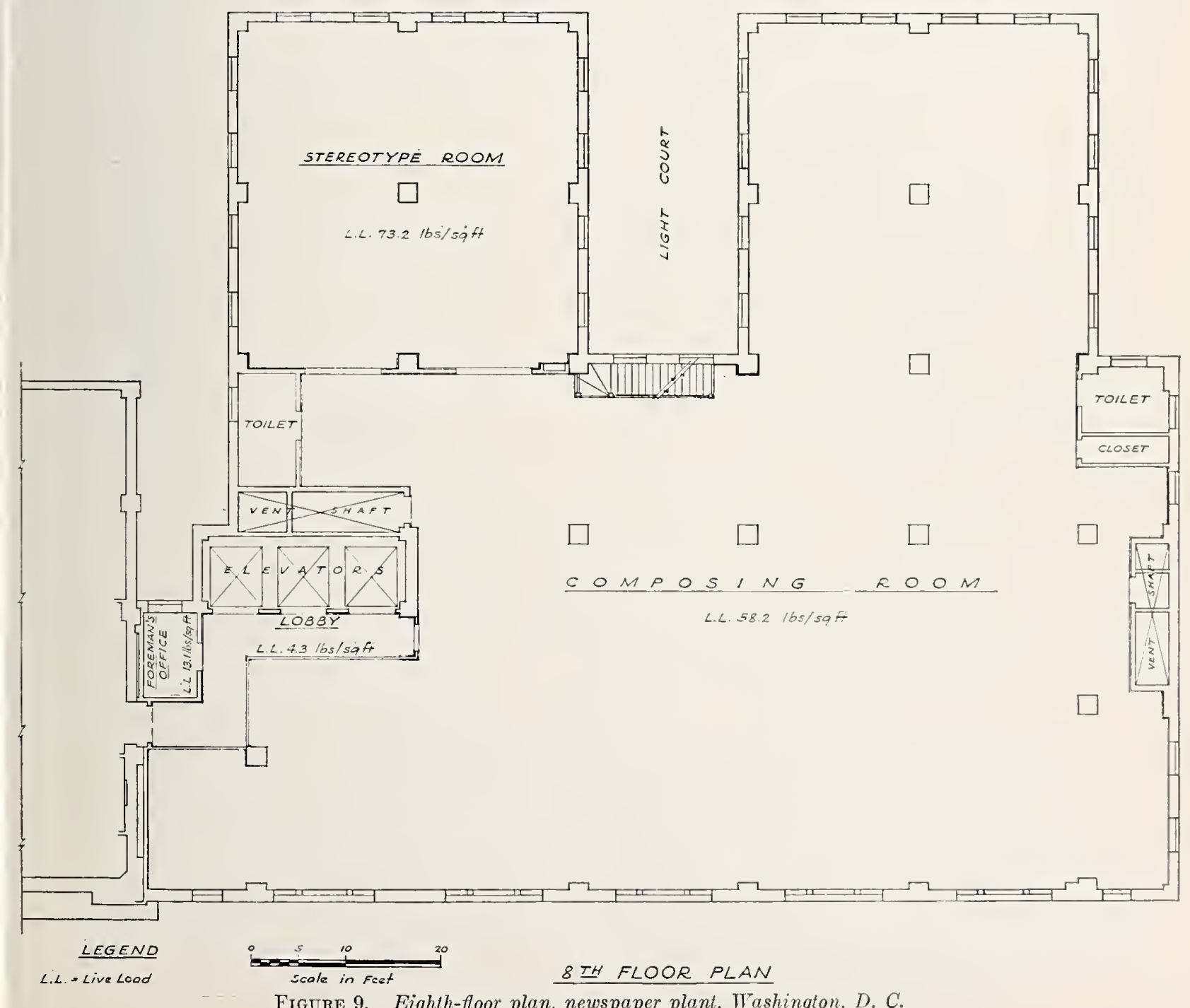


TABLE 18. Iariation in live loads in newspaper plant, Washington, D. C.

\begin{tabular}{|c|c|c|}
\hline Unit load & Area & $\begin{array}{l}\text { Portion of } \\
\text { total area }\end{array}$ \\
\hline $\begin{array}{l}l b / f t^{2} \\
0.0 \text { to } 4.9 \\
5.0 \text { to } 9.99 \\
10.0 \text { to } 14.9 \\
15.0 \text { to } 19.9 \\
20.0 \text { to } 24.9\end{array}$ & $\begin{array}{r}f+2 \\
6,125 \\
6,862 \\
20,709 \\
6,604 \\
15,229\end{array}$ & $\begin{array}{c}\text { Percent } \\
8.5 \\
9.5 \\
28.7 \\
9.1 \\
21.1\end{array}$ \\
\hline $\begin{array}{l}25.0 \text { to } 29.9 \\
30.0 \text { to } 34.9 \\
35.0 \text { to } 39.9 \\
40.0 \text { to } 44.9\end{array}$ & $\begin{array}{r}1,855 \\
1,540 \\
434 \\
-571\end{array}$ & $\begin{array}{l}2.6 \\
2.1 \\
\text { C. } 6 \\
.8\end{array}$ \\
\hline $\begin{array}{l}50.0 \text { to } 54.9 \\
55.0 \text { to } 59.9 \\
60.0 \text { to } 64.9 \\
65.0 \text { to } 69.9 \\
70.0 \text { to } 74.9\end{array}$ & $\begin{array}{r}1,794 \\
6,962 \\
154 \\
165 \\
2,423\end{array}$ & $\begin{array}{l}2.5 \\
9.6 \\
0.2 \\
.2 \\
3.3\end{array}$ \\
\hline $\begin{array}{l}75.0 \text { to } 79.9 \ldots \\
80.0 \text { to } 89.9 \ldots \\
90.0 \text { to } 94.9 \ldots\end{array}$ & 648 & $\frac{0.9}{.3}$ \\
\hline Total & 72,265 & $\begin{array}{l}100.0 \\
\times\end{array}$ \\
\hline
\end{tabular}
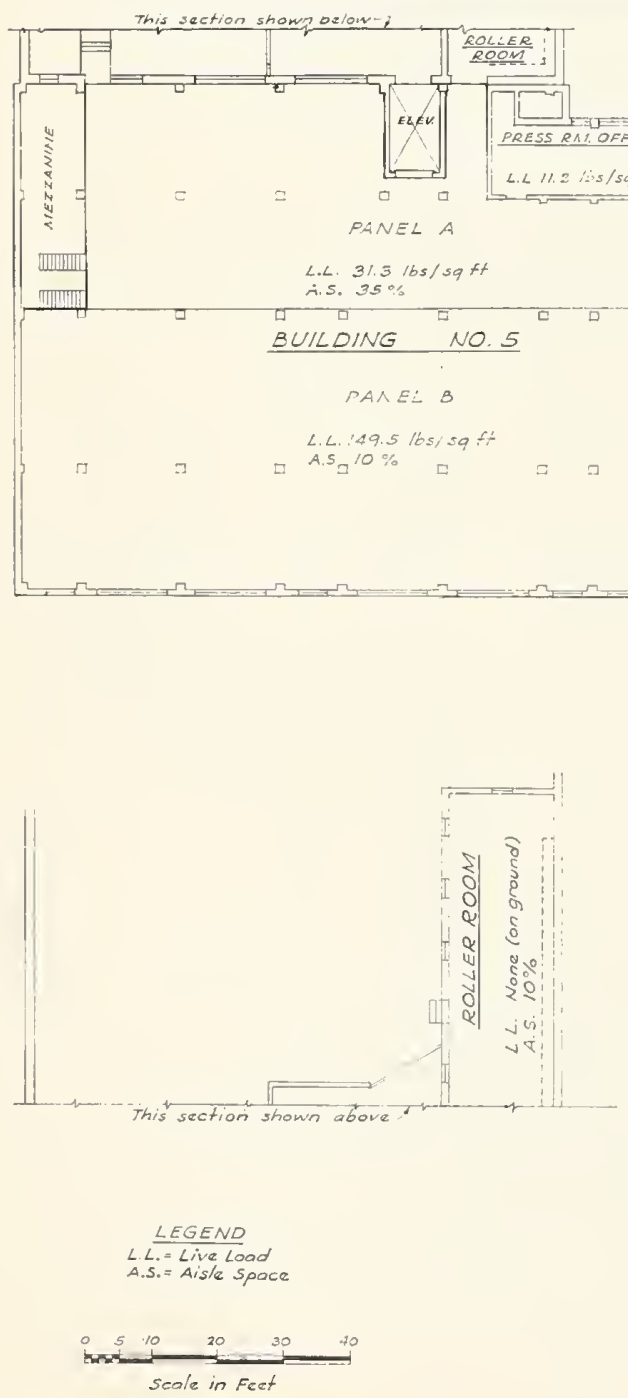

Printing Plant, Washington, D. C.:

Within each area the aisle space has been given as a percentage of the total area. This aisle space was usually kept open to provide for the transfer of large skids loaded with 20 to 30 reams of paper from one part of the plant to another.

Very heavy machinery loads on framed floors were found in this survey. In panel $B$ of the second floor of building 4 a press, together with its feeders and operators, and a 1/4-in. steel plate on the floor weighed $60,000 \mathrm{lb}$. An obsolete machinery lavout of this area gives reason to believe that this load occupied about one and one-half bays of the building, or $360 \mathrm{ft}^{2}$. This would mean a load of $168 \mathrm{lb} / \mathrm{ft}^{2}$. In panel $\mathrm{B}$ of the second floor of building 5 a rotary press, together with its crossfeed, operators, paper, and steel floor, weighed $151,400 \mathrm{lb}$. The old machine layout referred to above indicates that this load was probably carried on two full bays of floor, having an area of about $600 \mathrm{ft}^{2}$, making an average load on two bays of $252 \mathrm{lb} / \mathrm{ft}^{2}$. The results of the survey are given in tables 19 and 20 . Figure 10 shows a floor in the plant.

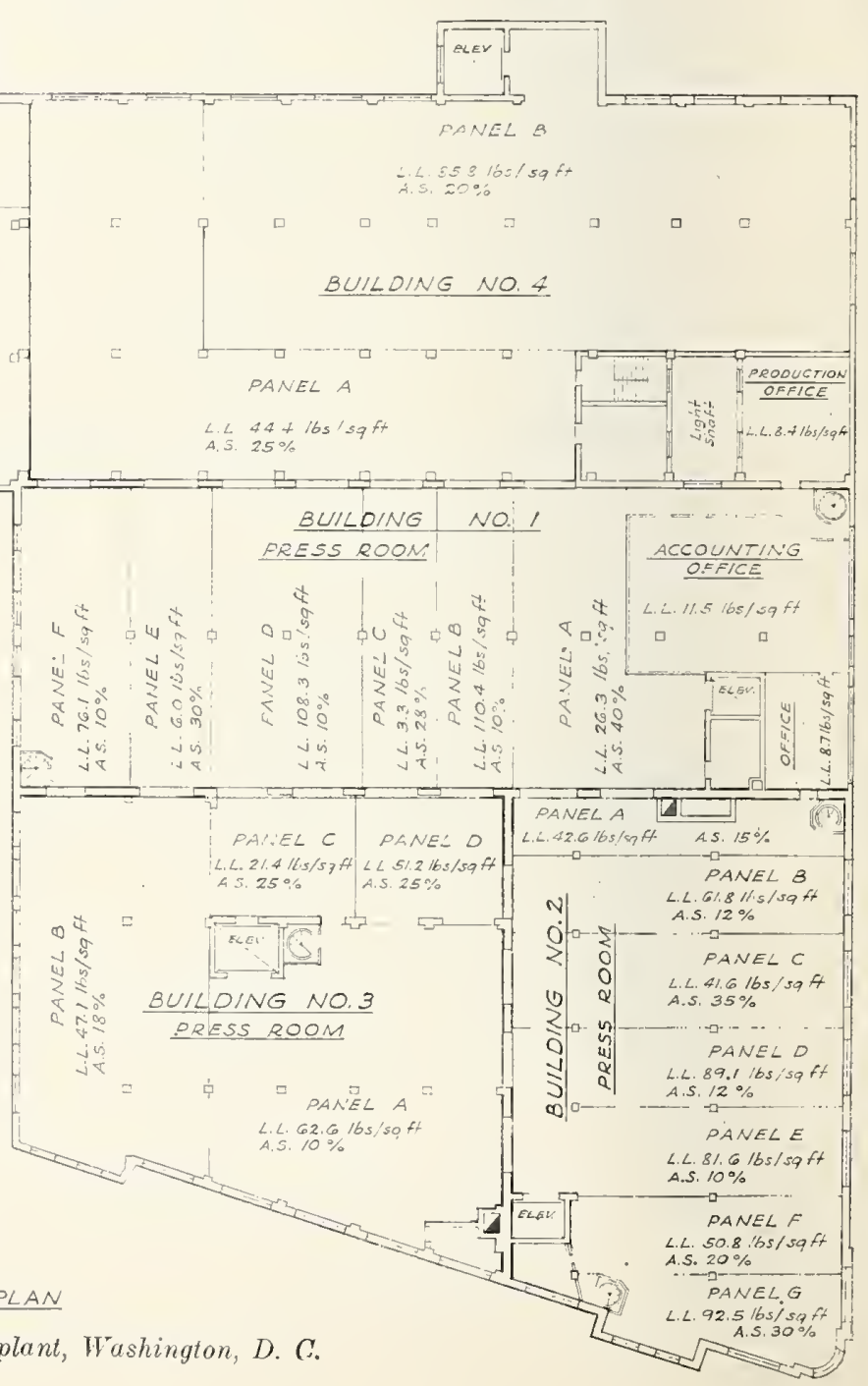


TA BLE 19. Live loads in prinling plant, W'ashington, D.C.

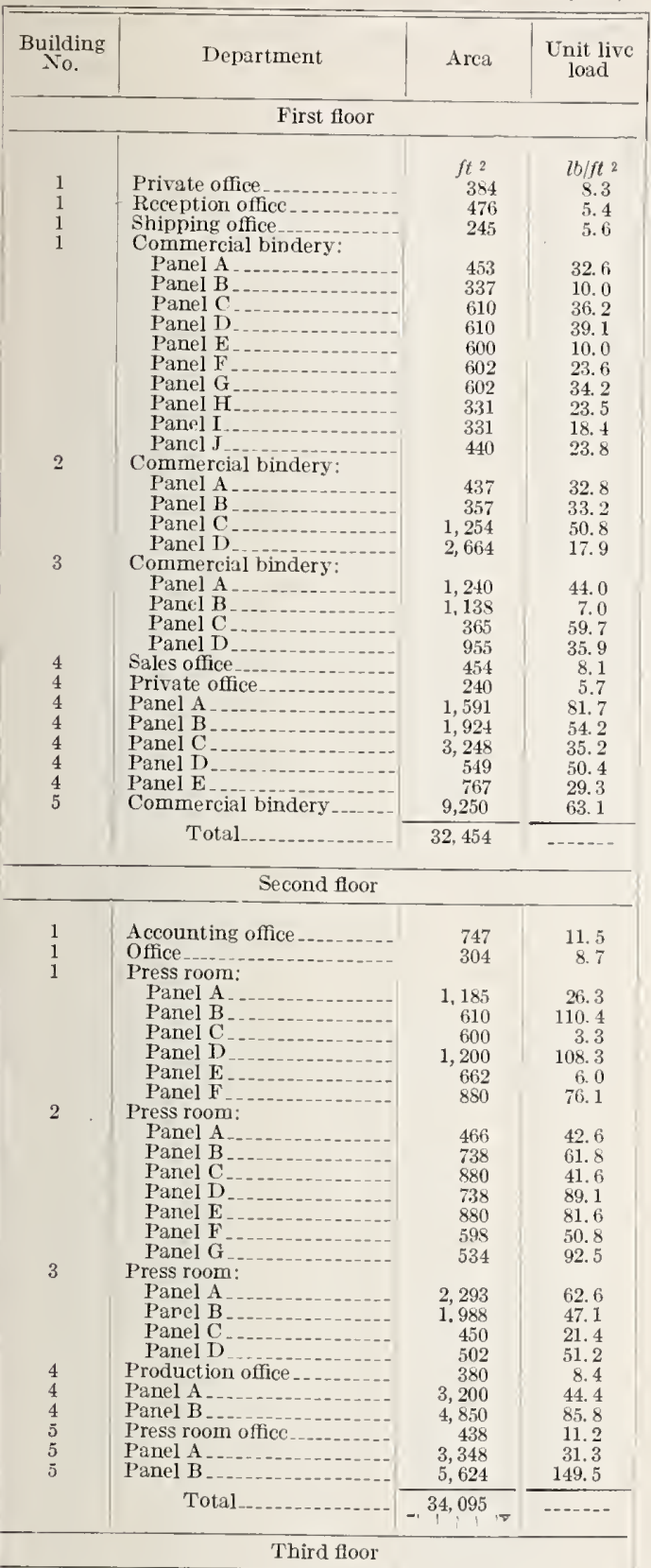

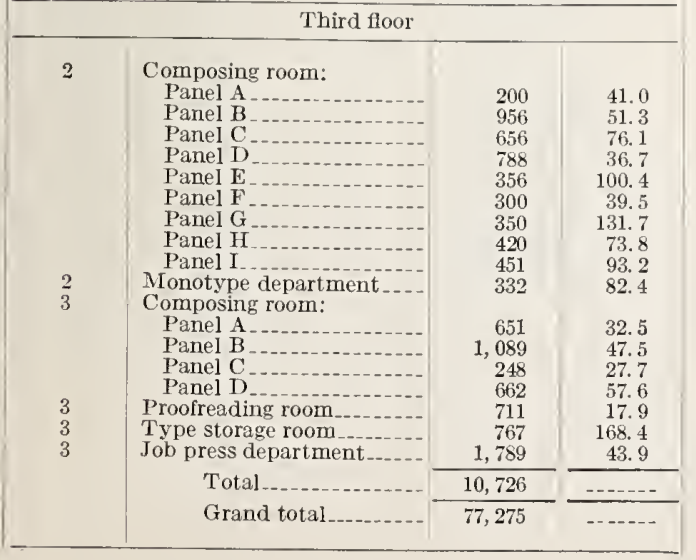

TABLE 20. Tariation in live loads in printing plant, I Washington, I). C.

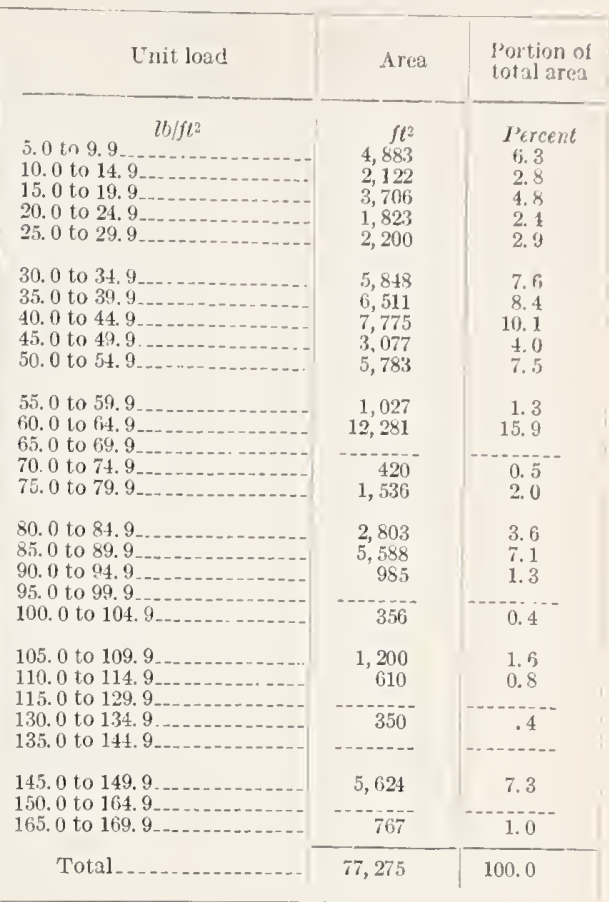

\section{Storage Occupancy}

The live loads on warehouse floors vary widely because of the many different kinds of materials stored. Building codes recommended by various organizations for national or regional use and building code standards give permissible minimum lesign load figures for light and heavy storage. In the case of light storage, the range is narrow, from 120 to $125 \mathrm{lb} / \mathrm{ft}^{2}$, while with heary storage a minimum ralue of $250 \mathrm{lb} / \mathrm{ft}^{2}$ is customary.

Methods of determining loading used in common for the twro cases survered were the noting of weights marked on barrels and other containers and computing the reight of persons at $150 \mathrm{lb}$ each for the number found in a giren area. Other methods are mentioned under the particular case to which they applr.

The resuits of the survey by the Public Buildings Administration appear in tables 21, 22, 23, and 24. Figures 11 and 12 show floor plans having the heariest unit loads in the case of this occupancy.

Warehouse, New York, $\Lambda^{\top}$. Y.:

Weights of various smaller items were establisher by weighing them directly. The walls and partitions that were carried by the floor slabs were measured and the weight computed.

In addition to the loads shown in the tables and drawing. there was one electric fork truck on each floor. The maximum live load caused by each truck is $6,000 \mathrm{lb}$, and in computing columm loads this truck may be assumed in any position designated as aisle space on the nine floors. 
TABLe 21. Live loads in warehouse, New York, N. Y.

\begin{tabular}{|c|c|c|c|c|c|c|c|c|c|c|c|}
\hline Bay & Area & $\begin{array}{l}\text { Base- } \\
\text { ment }\end{array}$ & $\begin{array}{l}\text { First } \\
\text { floor }\end{array}$ & $\begin{array}{l}\text { Second } \\
\text { floor }\end{array}$ & $\begin{array}{l}\text { Third } \\
\text { floor }\end{array}$ & $\begin{array}{c}\text { Fourth } \\
\text { floor }\end{array}$ & $\begin{array}{l}\text { Fifth } \\
\text { floor }\end{array}$ & $\begin{array}{l}\text { Sixth } \\
\text { floor }\end{array}$ & $\begin{array}{l}\text { Seventh } \\
\text { floor }\end{array}$ & $\begin{array}{c}\text { Eighth } \\
\text { floor }\end{array}$ & $\begin{array}{l}\text { Ninth } \\
\text { floor }\end{array}$ \\
\hline $\begin{array}{l}1 \\
2 \\
3 \\
4 \\
5\end{array}$ & $\begin{array}{l}f t^{2} \\
560 \\
526 \\
492 \\
454 \\
432\end{array}$ & $\begin{array}{r}l b / f t^{2} \\
1.1 \\
77.4 \\
156.6 \\
97.0 \\
\text { (a) }\end{array}$ & $\begin{array}{r}c b / f t^{2} \\
17.1 \\
22.5 \\
25.9 \\
32.3 \\
34.9\end{array}$ & $\begin{array}{r}\ell b / f t^{2} \\
46.3 \\
70.9 \\
40.2 \\
39.7 \\
\mathbf{1 4 . 4}\end{array}$ & $\begin{array}{r}\ell b / f t^{2} \\
93.8 \\
118.9 \\
0.0 \\
204.1 \\
105.8\end{array}$ & $\begin{array}{r}\text { lb/ft } 2 \\
109.4 \\
194.8 \\
80.3 \\
115.2 \\
86.5\end{array}$ & $\begin{array}{r}\text { bb/ft } 2^{2} \\
49.2 \\
73.4 \\
96.2 \\
92.5 \\
79.6\end{array}$ & $\begin{array}{r}\text { tblft } 2 \\
171.5 \\
49.6 \\
60.4 \\
82.3 \\
37.9\end{array}$ & $\begin{array}{l}l b / f t^{2} \\
118.7 \\
223.1 \\
256.6 \\
214.7 \\
175.8\end{array}$ & $\begin{array}{r}\text { Ub/ft }{ }^{2} \\
41.8 \\
82.7 \\
59.5 \\
14.6 \\
78.9\end{array}$ & $\begin{array}{r}t b / f t^{2} \\
73.8 \\
11.7 \\
181.0 \\
114.9 \\
46.4\end{array}$ \\
\hline $\begin{array}{r}6 \\
7 \\
8 \\
9 \\
10\end{array}$ & $\begin{array}{l}405 \\
396 \\
399 \\
399 \\
399\end{array}$ & \begin{tabular}{r}
\multicolumn{1}{c}{ (a) } \\
72.2 \\
225.7 \\
55.1 \\
36.6
\end{tabular} & $\begin{array}{r}7.8 \\
72.6 \\
42.5 \\
55.9 \\
26.0\end{array}$ & $\begin{array}{l}36.2 \\
64.4 \\
59.8 \\
87.7 \\
90.5\end{array}$ & $\begin{array}{l}60.6 \\
21.8 \\
41.7 \\
59.2 \\
23.0\end{array}$ & $\begin{array}{l}64.0 \\
17.1 \\
39.9 \\
63.1 \\
43.5\end{array}$ & $\begin{array}{l}64.2 \\
48.5 \\
55.5 \\
58.8 \\
52.1\end{array}$ & $\begin{array}{r}79.2 \\
16.8 \\
67.2 \\
67.7 \\
102.1\end{array}$ & $\begin{array}{r}88.4 \\
105.9 \\
122.8 \\
90.4 \\
71.7\end{array}$ & $\begin{array}{r}38.0 \\
57.2 \\
45.2 \\
43.3 \\
4.1\end{array}$ & $\begin{array}{l}72.7 \\
75.7 \\
62.9 \\
55.2 \\
37.3\end{array}$ \\
\hline $\begin{array}{l}11 \\
12 \\
13 \\
14 \\
15\end{array}$ & $\begin{array}{l}547 \\
405 \\
399 \\
399 \\
399\end{array}$ & $\begin{array}{l}136.1 \\
129.4 \\
134.6 \\
121.0 \\
178.8\end{array}$ & $\begin{array}{r}25.9 \\
66.4 \\
73.4 \\
106.2 \\
30.2\end{array}$ & $\begin{array}{r}65.6 \\
138.7 \\
80.9 \\
94.4 \\
44.1\end{array}$ & $\begin{array}{r}155.4 \\
127.9 \\
53.3 \\
139.6 \\
157.0\end{array}$ & $\begin{array}{r}147.3 \\
90.9 \\
80.1 \\
152.5 \\
105.3\end{array}$ & $\begin{array}{l}78.3 \\
46.9 \\
62.9 \\
70.2 \\
87.0\end{array}$ & $\begin{array}{r}135.9 \\
197.5 \\
150.8 \\
111.8 \\
50.1\end{array}$ & $\begin{array}{l}194.5 \\
151.1 \\
102.2 \\
174.1 \\
198.0\end{array}$ & $\begin{array}{r}139.4 \\
157.3 \\
6.4 \\
85.4 \\
86.7\end{array}$ & $\begin{array}{r}150.9 \\
197.0 \\
45.4 \\
83.0 \\
64.6\end{array}$ \\
\hline $\begin{array}{l}16 \\
17 \\
18 \\
19 \\
20\end{array}$ & $\begin{array}{r}396 \\
\text { b } 405 \\
406 \\
396 \\
400\end{array}$ & $\begin{array}{l}78.6 \\
94.5 \\
40.2 \\
92.2 \\
99.5\end{array}$ & $\begin{array}{r}37.0 \\
24.1 \\
23.4 \\
49.8 \\
106.9\end{array}$ & $\begin{array}{r}70.7 \\
87.3 \\
8.4 \\
50.9 \\
55.9\end{array}$ & $\begin{array}{r}83.0 \\
80.6 \\
42.6 \\
117.4 \\
136.8\end{array}$ & $\begin{array}{r}179.7 \\
74.8 \\
43.4 \\
122.9 \\
110.4\end{array}$ & $\begin{array}{r}180.2 \\
72.4 \\
27.4 \\
132.3 \\
123.5\end{array}$ & $\begin{array}{r}60.6 \\
131.1 \\
63.8 \\
105.2 \\
92.4\end{array}$ & $\begin{array}{r}212.4 \\
116.1 \\
18.4 \\
160.3 \\
160.9\end{array}$ & $\begin{array}{l}72.2 \\
53.6 \\
16.2 \\
28.7 \\
45.4\end{array}$ & $\begin{array}{l}86.0 \\
99.1 \\
58.7 \\
46.0 \\
96.3\end{array}$ \\
\hline $\begin{array}{l}21 \\
22 \\
23 \\
24 \\
25\end{array}$ & $\begin{array}{l}400 \\
400 \\
406 \\
411 \\
405\end{array}$ & $\begin{array}{r}88.5 \\
93.2 \\
96.1 \\
213.7 \\
137.6\end{array}$ & $\begin{array}{l}48.4 \\
55.0 \\
54.8 \\
65.7 \\
62.5\end{array}$ & $\begin{array}{r}62.3 \\
80.8 \\
63.0 \\
90.3 \\
156.5\end{array}$ & $\begin{array}{r}71.3 \\
143.5 \\
228.3 \\
198.4 \\
154.1\end{array}$ & $\begin{array}{r}133.2 \\
122.3 \\
139.3 \\
97.5 \\
134.3\end{array}$ & $\begin{array}{r}92.1 \\
\mathbf{1 8 4 . 2} \\
59.1 \\
155.1 \\
81.6\end{array}$ & $\begin{array}{l}111.7 \\
150.0 \\
112.1 \\
163.4 \\
194.4\end{array}$ & $\begin{array}{r}67.9 \\
57.6 \\
50.9 \\
155.3 \\
155.1\end{array}$ & $\begin{array}{r}53.1 \\
6.8 \\
158.6 \\
229.5 \\
89.3\end{array}$ & $\begin{array}{r}39.7 \\
53.3 \\
139.1 \\
75.0 \\
73.4\end{array}$ \\
\hline $\begin{array}{l}26 \\
27 \\
28\end{array}$ & $\begin{array}{l}405 \\
405 \\
402\end{array}$ & $\begin{array}{l}79.8 \\
78.5 \\
24.8\end{array}$ & $\begin{array}{r}68.7 \\
118.2 \\
55.6\end{array}$ & $\begin{array}{r}85.6 \\
126.4 \\
135.6\end{array}$ & $\begin{array}{r}141.6 \\
33.8 \\
56.3\end{array}$ & $\begin{array}{r}100.7 \\
124.6 \\
66.9\end{array}$ & $\begin{array}{r}106.0 \\
78.9 \\
72.2\end{array}$ & $\begin{array}{l}131.1 \\
146.0 \\
105.4\end{array}$ & $\begin{array}{r}157.4 \\
100.3 \\
98.9\end{array}$ & $\begin{array}{r}80.2 \\
97.8 \\
101.7\end{array}$ & $\begin{array}{l}65.0 \\
75.3 \\
58.2\end{array}$ \\
\hline
\end{tabular}

- Bays 5 and 6 in basement were not surveyed.

b Area of bay 17 in basement, $200 \mathrm{ft}^{2}$; in all floors above, $405 \mathrm{ft}^{2}$.

TABLE 22. Variation in live loads in warehouse, New York, N. Y.

\begin{tabular}{|c|c|c|}
\hline Unit load & Area & $\begin{array}{l}\text { Portion of } \\
\text { total area }\end{array}$ \\
\hline $\begin{array}{l}l b / f t^{2} \\
0.0 \text { to } 4.9 \\
5.0 \text { to } 9.9 \\
10.0 \text { to } 14.9 \\
15.0 \text { to } 19.9 \\
20.0 \text { to } 24.9\end{array}$ & $\begin{array}{l}f t^{2} \\
1,451 \\
1,610 \\
432 \\
2,164 \\
2,534\end{array}$ & $\begin{array}{c}\text { Percent } \\
1.2 \\
1.4 \\
0.4 \\
1.8 \\
2.2\end{array}$ \\
\hline $\begin{array}{l}25.0 \text { to } 29.9 \\
30.0 \text { to } 34.9 \\
35.0 \text { to } 39.9 \\
40.0 \text { to } 44.9 \\
45.0 \text { to } 49.9\end{array}$ & $\begin{array}{l}2,240 \\
1,690 \\
3,689 \\
4,265 \\
5,269\end{array}$ & $\begin{array}{l}1.9 \\
1.4 \\
3.1 \\
3.6 \\
4.5\end{array}$ \\
\hline $\begin{array}{l}50.0 \text { to } 54.9 \\
55.0 \text { to } 59.9 \\
60.0 \text { to } 64.9 \\
65.0 \text { to } 69.9 \\
70.0 \text { to } 74.9\end{array}$ & $\begin{array}{l}3,610 \\
6,899 \\
5,712 \\
3,773 \\
6,815\end{array}$ & $\begin{array}{l}3.1 \\
5.9 \\
4.9 \\
3.2 \\
5.8\end{array}$ \\
\hline $\begin{array}{l}75.0 \text { to } 79.9 \\
80.0 \text { to } 84.9 \\
85.0 \text { to } 89.9 \\
90.0 \text { to } 94.9 \\
95.0 \text { to } 99.9\end{array}$ & $\begin{array}{l}5,165 \\
4,680 \\
4,444 \\
4,823 \\
3,775\end{array}$ & $\begin{array}{l}4.4 \\
4.0 \\
3.8 \\
4.1 \\
3.2\end{array}$ \\
\hline $\begin{array}{l}100.0 \text { to } 104.9 \\
105.0 \text { to } 109.9 \\
110.0 \text { to } 114.9 \\
115.0 \text { to } 119.9 \\
120.0 \text { to } 124.9\end{array}$ & $\begin{array}{l}2,010 \\
3,789 \\
2,585 \\
2,746 \\
2,399\end{array}$ & $\begin{array}{l}1.7 \\
3.2 \\
2.2 \\
2.3 \\
2.0\end{array}$ \\
\hline
\end{tabular}

TABLE 22. Variation in live loads in warehouse, New York, N. Y.-Continued

\begin{tabular}{|c|c|c|}
\hline Unit load & Area & $\begin{array}{l}\text { Portion of } \\
\text { total area }\end{array}$ \\
\hline $\begin{array}{l}l \mathrm{l} / f t^{2} \\
125.0 \text { to } 129.9 \\
130.0 \text { to } 134.9 \\
135.0 \text { to } 139.9 \\
140.0 \text { to } 144.9 \\
145.0 \text { to } 149.9\end{array}$ & $\begin{array}{r}f t^{2} \\
1,215 \\
2,410 \\
4,464 \\
1,259 \\
952\end{array}$ & $\begin{array}{l}\text { Percent } \\
1.0 \\
2.1 \\
3.8 \\
1.1 \\
0.8\end{array}$ \\
\hline $\begin{array}{l}150.0 \text { to } 154.9 \\
155.0 \text { to } 159.9 \\
160.0 \text { to } 164.9 \\
165.0 \text { to } 169.9 \\
170.0 \text { to } 174.9\end{array}$ & $\begin{array}{r}2,555 \\
4,286 \\
1,207 \\
959\end{array}$ & $\begin{array}{c}2.2 \\
3.7 \\
1.0 \\
0.8\end{array}$ \\
\hline $\begin{array}{l}175.0 \text { to } 179.9 \\
180.0 \text { to } 181.9 \\
185.0 \text { to } 189.9 \\
190.0 \text { to } 194.9\end{array}$ & $\begin{array}{c}1,227 \\
1,288 \\
1,478 \\
1,620\end{array}$ & \begin{tabular}{c}
1.0 \\
1.1 \\
\hdashline 1.3 \\
1.4
\end{tabular} \\
\hline $\begin{array}{l}200.0 \text { to } 204.9 \ldots \\
205.0 \text { to } 209.9 \ldots \\
210.0 \text { to } 214.9 \ldots \\
215.0 \text { to } 219.9 \ldots \\
220.0 \text { to } 224.9 \ldots\end{array}$ & $\begin{array}{r}454 \\
807 \\
526\end{array}$ & $\begin{array}{c}0.4 \\
-7^{-} \\
.5\end{array}$ \\
\hline $\begin{array}{l}225.0 \text { to } 229.9 \\
230.0 \text { to } 239.9 \\
240.0 \text { to } 244.9 \\
245.0 \text { to } 254.9 \\
255.0 \text { to } 259.9\end{array}$ & 1,216 & $\begin{array}{c}1.0 \\
0.4 \\
0.4\end{array}$ \\
\hline Total & 117,438 & 100.0 \\
\hline
\end{tabular}




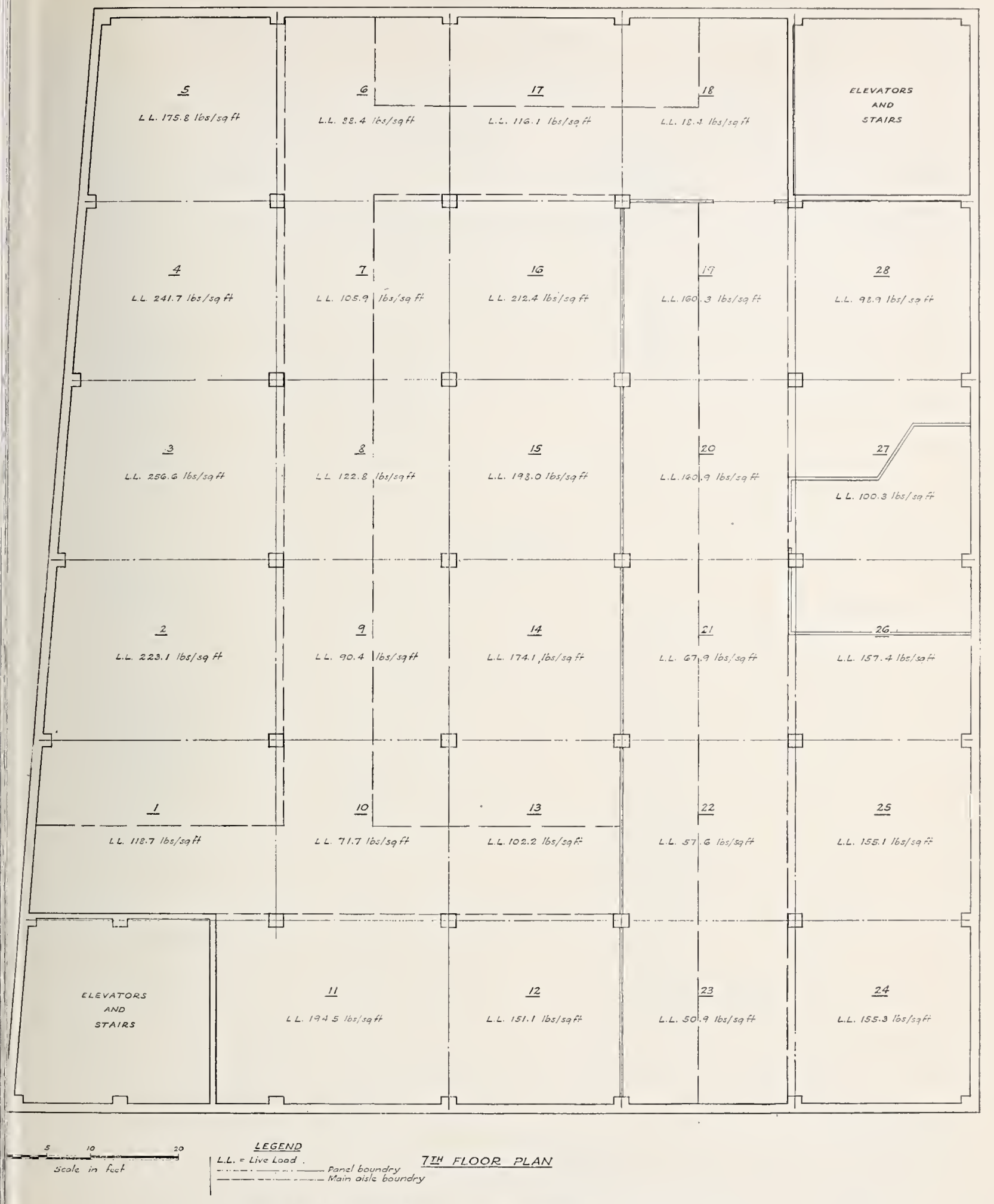

Figure 11. Seventh-floor plan, warehouse, New York, N. Y. 


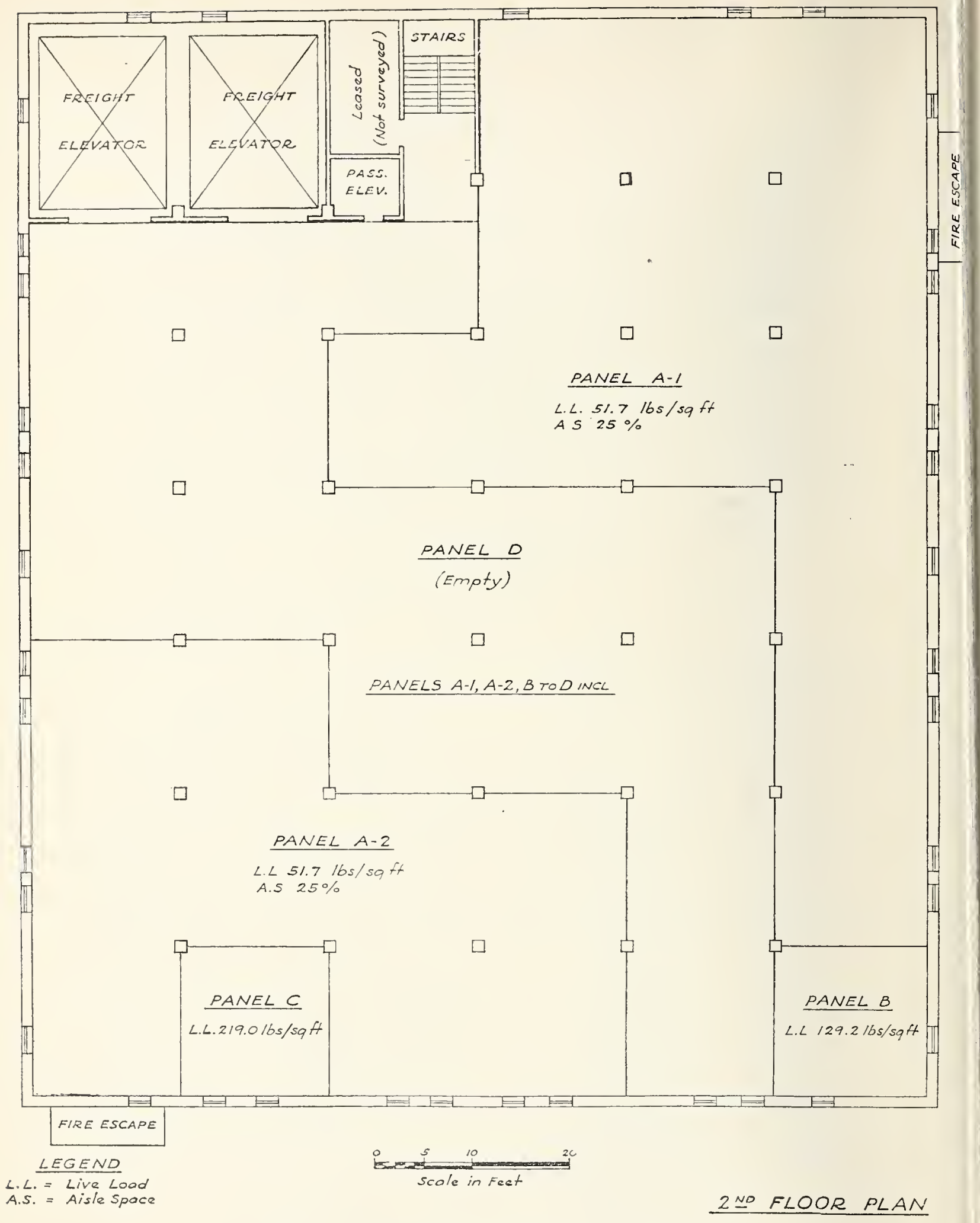

Figure 12. Second-floor plan, warehouse, Washington, D. C. 
Warehouse, Washington, D. C.:

A general merchandise warehouse and its offices were the subject of this survey.

Weights of typical items, such as furniture, packages of canned fruit, canned vegetables, cooking oil, etc., were established by weighing representative pieces. Some items, such as built-in construction, that could not be weighed were measured and the weight computed.

TABLE 23. Live loads in warehouse, Washington, D. C.

\begin{tabular}{|c|c|c|}
\hline Department & Area & $\begin{array}{l}\text { Unit live } \\
\text { load }\end{array}$ \\
\hline \multicolumn{3}{|l|}{ First floor } \\
\hline $\begin{array}{l}\text { Private offiee. } \\
\text { General offiee. } \\
\text { Vestibule. } \\
\text { Sales offiee... } \\
\text { Storage room A } \\
\text { Panel A } \\
\text { Panel B } \\
\text { Panel C } \\
\text { Panel D } \\
\text { Panel E } \\
\text { Panel F } \\
\text { Panel G. }\end{array}$ & $\begin{array}{c}f t 2 \\
192 \\
1,167 \\
236 \\
653 \\
95 \\
234 \\
234 \\
684 \\
225 \\
410 \\
2,640 \\
270\end{array}$ & $\begin{array}{r}\imath b / f t^{2} \\
10.2 \\
8.8 \\
3.2 \\
9.5 \\
24.2 \\
22.9 \\
29.8 \\
35.3 \\
181.5 \\
27.5 \\
8.1 \\
0.0\end{array}$ \\
\hline - & 7,040 & -...... \\
\hline \multicolumn{3}{|l|}{ Second floor } \\
\hline $\begin{array}{l}\text { Panels A-1 and A-2 } \\
\text { Panel B } \\
\text { Panel C } \\
\text { Panel D. }\end{array}$ & $\begin{array}{r}5,100 \\
221 \\
231 \\
3,425\end{array}$ & $\begin{array}{r}51.7 \\
129.2 \\
219.0 \\
0.0\end{array}$ \\
\hline Total_... & 8,977 & $-\ldots-$ \\
\hline \multicolumn{3}{|l|}{ Third floor } \\
\hline 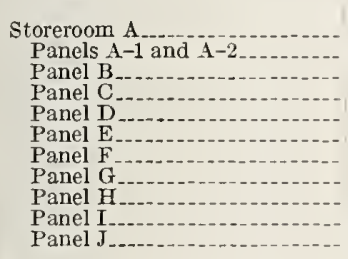 & $\begin{array}{r}95 \\
3,295 \\
468 \\
234 \\
710 \\
473 \\
238 \\
238 \\
239 \\
958 \\
1,763\end{array}$ & $\begin{array}{r}2.1 \\
54.9 \\
121.8 \\
63.0 \\
11.8 \\
24.4 \\
75.8 \\
34.6 \\
280.7 \\
0.0 \\
.0\end{array}$ \\
\hline Total & 8,711 & -..... \\
\hline \multicolumn{3}{|l|}{ Fourth floor } \\
\hline $\begin{array}{l}\text { Storeroom A } \\
\text { Panel A } \\
\text { Panel B.......... } \\
\text { Panel C }\end{array}$ & $\begin{array}{r}95 \\
7,600 \\
474 \\
589\end{array}$ & $\begin{array}{r}41.1 \\
77.4 \\
303.5 \\
0.0\end{array}$ \\
\hline Total.. & 8,758 & $-\ldots-1$ \\
\hline \multicolumn{3}{|l|}{ Fifth floor } \\
\hline $\begin{array}{l}\text { Storeroom B. } \\
\quad \text { Panel A } \\
\text { Panel B.... }\end{array}$ & $\begin{array}{r}650 \\
7,760 \\
589\end{array}$ & $\begin{array}{r}118.8 \\
95.1 \\
0.0\end{array}$ \\
\hline Total & 8,999 & $-\cdots$ \\
\hline \multicolumn{3}{|l|}{ Sixth floor } \\
\hline $\begin{array}{l}\text { Storeroom A } \\
\text { Storeroom B-m } \\
\text { Storeroom C } \\
\text { Panel A } \\
\text { Panel B } \\
\text { Panel C }\end{array}$ & $\begin{array}{r}95 \\
255 \\
518 \\
6,890 \\
958 \\
589\end{array}$ & $\begin{array}{r}20.2 \\
18.6 \\
40.0 \\
33.6 \\
0.0 \\
.0\end{array}$ \\
\hline Total & 9,305 & - \\
\hline
\end{tabular}

TABLE 23. Live loads in warehouse, Washington, I). C.Continued

\begin{tabular}{|c|c|c|}
\hline 1)epartment & A rea & $\begin{array}{c}\text { Unit live } \\
\text { load }\end{array}$ \\
\hline \multicolumn{3}{|c|}{ Seventh floor } \\
\hline $\begin{array}{l}\text { Storeroom A } \\
\text { Storeroom B } \\
\text { Storeroom C } \\
\text { Panel A } \\
\text { Panel B... }\end{array}$ & $\begin{array}{r}f t^{2} \\
95 \\
184 \\
589 \\
7,818 \\
589\end{array}$ & $\begin{array}{r}l b / f t^{2} \\
70.7 \\
6.6 \\
13.2 \\
30.4 \\
0.0\end{array}$ \\
\hline Total & 9,305 & -...... \\
\hline \multicolumn{3}{|c|}{ Eighth floor } \\
\hline $\begin{array}{l}\text { Storeroom } \mathrm{A} \\
\text { Panels } \mathrm{A}-1 \text { and } \mathrm{A}-2 \\
\text { Panel } \mathrm{B}- \\
\text { Panel } \mathrm{C} \\
\text { Panel D.... }\end{array}$ & $\begin{array}{r}95 \\
2,213 \\
4,287 \\
713 \\
754\end{array}$ & $\begin{array}{r}18.8 \\
22.3 \\
21.1 \\
39.3 \\
0.0\end{array}$ \\
\hline Total. & 8,062 & - \\
\hline Grand total ..... & 69,157 & $\ldots$ \\
\hline
\end{tabular}

TABLE 24. Iariation in live loads in warehouse, Washington, D.C.

\begin{tabular}{|c|c|c|}
\hline Unit load & Area & $\begin{array}{l}\text { Portion of } \\
\text { total area }\end{array}$ \\
\hline $\begin{array}{l}l b / f t^{2} \\
0.0 \text { to } 4.99 \\
5.0 \text { to } 9.9 \\
10.0 \text { to } 14.9 \\
15.0 \text { to } 19.9 \\
20.0 \text { to } 24.9\end{array}$ & $\begin{array}{r}f t^{2} \\
10,815 \\
4,644 \\
1,491 \\
350 \\
7,397\end{array}$ & $\begin{array}{c}\text { Percent } \\
15.7 \\
6.7 \\
2.2 \\
0.5 \\
10.7\end{array}$ \\
\hline $\begin{array}{l}25.0 \text { to } 29.9 \\
30.0 \text { to } 34.9 \\
35.0 \text { to } 39.9 \\
40.0 \text { to } 44.9 \\
45.0 \text { to } 49.9\end{array}$ & $\begin{array}{r}644 \\
14,976 \\
1,397 \\
613 \\
---\end{array}$ & $\begin{array}{r}0.9 \\
21.7 \\
2.0 \\
0.9 \\
\end{array}$ \\
\hline $\begin{array}{l}50.0 \text { to } 54.9 \\
55.0 \text { to } 59.9 \\
60.0 \text { to } 64.9 \\
70.0 \text { to } 69.9\end{array}$ & 8,395 & \begin{tabular}{c}
12.2 \\
\hdashline .3 \\
0.3
\end{tabular} \\
\hline $\begin{array}{l}75.0 \text { to } 79.9 \\
80.0 \text { to } 94.9 \\
95.0 \text { to } 99.9 \\
100.0 \text { to } 114.9 \\
115.0 \text { to } 119.9\end{array}$ & $\begin{array}{c}7,838 \\
7,760 \\
650\end{array}$ & \begin{tabular}{c}
11.4 \\
\hdashline 11.2 \\
0.9
\end{tabular} \\
\hline $\begin{array}{l}120.0 \text { to } 124.9 \\
125.0 \text { to } 129.9 \\
130.0 \text { to } 179.9 \\
180.0 \text { to } 184.9 \\
185.0 \text { to } 214.9\end{array}$ & $\begin{array}{r}468 \\
221 \\
---- \\
-255 \\
----\end{array}$ & $\begin{array}{c}.7 \\
.3 \\
-3 \\
-3 \\
-\cdot-2\end{array}$ \\
\hline $\begin{array}{l}215.0 \text { to } 219.9 \\
220.0 \text { to } 279.9 \\
280.0 \text { to } 284.9 \\
285.0 \text { to } 299.9 \\
300.0 \text { to } 304.9\end{array}$ & $\begin{array}{r}231 \\
-239 \\
-474\end{array}$ & $\begin{array}{c}.3 \\
-3 \\
0.7\end{array}$ \\
\hline Total. & 69,157 & 100.0 \\
\hline
\end{tabular}

\section{Variation in Loading}

The data show fairly large differences in loading on different areas within the same occupancy. In some cases, such as a library or storage room in office occupancy, it may be possible to anticipate and provide for unusually heary loads. In others, as in department stores, there may be frequent shifts of goods from one part of a floor to another or from floor to floor in response to changes in 
sales policy, seasonal demands, or other considerations, with the result that unit loads on given areas may change several hundred percent.

It is apparent that there is a problem in the selection of a representative minimum unit-load value for each typical occupancy and in the determination of a reasonable reduced unit-load value for a large area supported by a given structural member. In the case of a small area, the total load will be the minimum unit load multiplied by the area; but in the case of larger areas, a reduction is considered permissible for loads carried by columns and girders, and sometimes beams, based on recognition of the fact that in many occupancies such members seldom, if ever, are loaded to the extent of the unit live load multiplied by the total area supported. A method of reduction that is gaining wide acceptance in building codes is as follows [5]:

For live loads of 100 pounds or less per square foot, the design live load on any member supporting 150 $\mathrm{lb} / \mathrm{ft}^{2}$ or more may be reduced at the rate of 0.08 percent per square foot of area supported by the member, except that no reduction shall be made for areas to be oceupied as places of public assembly. The reduction shall exceed neither $R$ as determined by the following formula, nor 60 percent:

$$
R=100 \times \frac{D+L}{4.33 L}
$$

in which

$$
\begin{aligned}
& R=\text { reduction in percent } \\
& D=\text { dead load per square foot of area supported } \\
& \quad \text { by the member } \\
& L=\text { design live load per square foot of area sup- } \\
& \quad \text { ported by the member. }
\end{aligned}
$$

For live loads exceeding $100 \mathrm{lb} / \mathrm{ft}^{2}$, no reduction shall be made, except that the design live loads on columns may be reduced 20 percent.

The design load $L$ in the above formula is the highest average live load that it is proposed to permit on room-sized areas (specifically, areas up to $150 \mathrm{ft}^{2}$. An individual member carrying less than $150 \mathrm{ft}^{2}$ of floor would be designed for $L$. It can also be thought of as the allowable load for which the floor should be posted, in situations where posting is advisable.

High concentrations of load on very small areas, such as safes, are ordinarily provided for in building codes by a requirement that the floor must be capable of supporting a $2,000-\mathrm{Jb}$ load on a specified limited area at any location.

The significance of $R$ is that it is a limiting value of the reduction in terms of the live load and dead load such that, if the entire structure were loaded at the rate of $L$, no part would be overstressed by more than 30 percent.

The effect of the foregoing method of reducing design live loads can be illustrated by an example. Assume, for instance, that $L$ equals $100 \mathrm{lb} / \mathrm{ft}^{2}, D$ equals $100 \mathrm{lb} / \mathrm{ft}^{2}$, and that the floor bays are $20 \mathrm{ft}$ sq with the slabs spanning in one direction. The slabs would be designed for a live load of 100 $\mathrm{lb} / \mathrm{ft}^{2}$. The beams and the top stories of columns carrying $400 \mathrm{ft}^{2}$ each would be designed for 68 $\mathrm{lb} / \mathrm{ft}^{2}$. The lower stories of the columns would be designed for $54 \mathrm{lb} / \mathrm{ft}^{2}$, which is the maximum reduction permitted by the formula. It is of interest to compare this value with the range of values found for large areas in the departmentstore surveys in this report.

\section{Discussion}

Safe building design requires the use of a combination of assumed loads and working stresses which will result in structures that will not be seriously overstressed in any part by the imposed loads.

The data obtained in the survey made by the Public Buildings Administration afford a chance of seeing in specific instances what the result of any given combination of live loads and stresses would liave been. The more extensive and detailed such surveys are for a given occupancy, the firmer are the conclusions to be drawn from them. The data represent not the most severe loading possible, but the actual average loading in each case on a given area of a given occupancy. It appears from the data that a large percentage of the floor area in most occupancies is much less heavily loaded than the most heavily loaded bay. This suggests possible further economies in the design of members carrying large floor areas. However, the data available are still small in quantity and there are conditions of local load concentrations that must not be overlooked. With additional information gained from further surveys, it should be possible to answer more definitely several questions, among them how representative are the minimum live-load assumptions now given in building codes recommended by various organizations for national or regional use.

Consideration of the use of the data presented herein leads to several suggestions for future surveys that would clear up some of the questions left open.

One such survey would be an investigation of the actual loads in department stores during the Christmas season. This could be done by selecting several bays that appeared to be busiest, measuring the areas therein that might be occupied by people, and subsequently counting the number of people in those areas from time to time during the Christmas shopping period. Photographs could also record the extent of crowding.

Another survey, or series of surveys, which would not be unduly expensive, would be to determine the most heavily loaded single bay of a floor in each of a number of manufacturing plants. This could be done by selecting the five bays that appeared, upon casual examination, to be most heavily loaded, and surveying those five bays in detail.

A third type of survey would consist of checking the loading in a number of occupancies where no published information is available that is less than twenty years old. Conditions may have clianged 
to such an extent as to make a redetermination of values for use in assumptions for design desirable.

If it is found through additional work in this field that substantial reductions can be made in minimum assumed unit loads for some occupancies and that significant economies in construction are indicated through use of the lowered figures, there are still some considerations that merit attention. One of these is the fact that local authorities are not always in a position to keep a close chesk on changes in occupancy and, consequently, buildings may become overloaded although they were safe as designed for their original tenant. Another consideration is that it may be economically desirable to design for such future changes through using a bigher unit load figure than is absolutely necessary because it then becomes possible to accommodate a greater variety of occupancies as time goes on. In this case, the problem becomes one of weighing initial savings against the advantages of flexibility of use over a long period.

In any event, it is important to have available a reasonably complete and up-to-date set of figures that can serve as the basis for engineering design.

\section{Selected References}

[1] The amount of possible strain on a crowded floor American Arehitect and Juilding News, p. 34 (April 15,1893 ).

[2] A study of offiee-building live loads, Eng News-Record, 90, 584 (Mareh 29, 1923).

[3] Minimum live loads allowable for use in design of buildings, Report of the Department of Commerce Building Code Committee, Nov, 1, 1924. (out of print)

[4] John W. Dunham, Design live loads in buildings, Trans. Am Soe Civil Engrs. 112, 725 (1947).

[5] Ameriean standard building code requirements for minimum design loads in buildings and other structures, NBS Miscellancous Publications M179 (1945).

[6] Report of Subeommittee on Fire-Resistance Classifications of the Central Housing Committee on Researeh, Design, and Construetion, Fire-resistanee elassifications of building constructions, NBS Building Materials and Struetures Report BMS92 (1942).

Washington, January 24, 1952. 


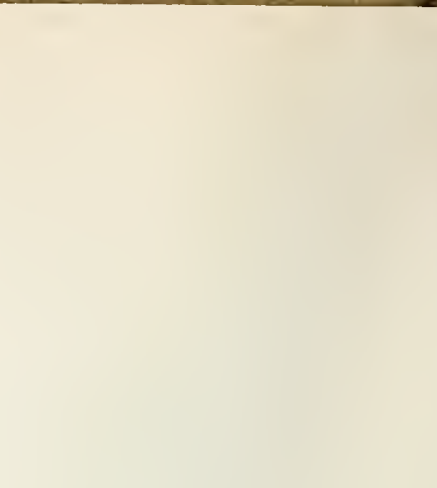




\section{BUILDING MATERIALS AND STRUCTURES REPORTS}

\section{[Continued from cover page II]}

BMS36 Structural Properties of Wood-Frame Wall, Partition, Floor, and Roof Constructions With "Red Stripe" Lath Sponsored by The Weston Paper and Manufacturing Co_._ 10k

BMS38 Structural Properties of Two "Dunstone" Wall Constructions Sponsored by the W. E. Dunn Manufacturing Co

BMS39 Structural Properties of a Wall Construction of "Pfeifer Units" Sponsored by the Wisconsin Units Co a Wall Construction of

BMS44 Surface Treatment of Steel Prior to Painting

BMS47 Structural Properties of Prefabricated Wood-Frame Constructions for Walls, Partitions, and Floors Sponsored by American Houses, Inc.

BMS48 Structural Properties of "Precision-Built" Frame Wall and Partition Constructions Sponsored by the Homasote Co

BMS50 Stability of Fiber Building Boards as Determined by Accelerated Aging

BMS51 Structural Properties of "Tilecrete Type A" Floor Construction Sponsored by the Tilecrete Co

BMS52

BMS53

Effect of Ceiling Insulation Upon Summer Comfort
Structural Properties of a Masonry Wall Construction of "Munlock Dry Wall Brick" Sponsored by the Munlock Engineering Co

BMS54 Effect of Soot on the Rating of an Oil-Fired Heating Boiler

BMS55 Effects of Wetting and Drying on the Permeability of Masonry Walls...

BMS58 Strength of Soft-Soldered Joints in Copper Tubing

BMS60 Strength, Absorption, and Resistance to Laboratory Freezing and Thawing of Building Bricks Produced in the United States .

BMS62 Structural Properties of a Precast Joist Concrete Floor Construction Sponsored by the Portland Cement Association

BMS63 Moisture Condensation in Building Walls

BMS65 Methods of Estimating Loads in Plumbing Systems

BMS66 Plumbing Manual

BMS67 Structural Properties of "Mu-Steel" Prefabricated Sheet-Steel Constructions for Walls, Partitions, Floors, and Roofs, Sponsored by Herman A. Mugler

Performance Test for Floor Coverings for Use in Low-Cost Housing: Part 3.

Stability of Fiber Sheathing Boards as Determined by Accelerated Aging

Asphalt-Prepared Roll Roofings and Shingles....

Fire Tests of Wood- and Metal-Framed Partitions "Precision-Built, Jr." Prefabricated Wood-Frame Wall Construction Sponsored by the Homasote Co

BMS68

BMS70

BMS71

BMS72

BMS73

BMS74

Indentation Characteristics of Floor Coverings
Structural and Heat-Transfer Properties of "U. S. S. Panelbilt" Prefabricated Sheet-
Steel Constructions for Walls, Partitions, and Roofs Sponsored by the Tennessee Coal, Iron \& Railroad Co

BMS75

Survey of Iron \& Railroad Co

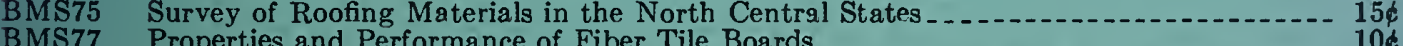

BMS77 Properties and Performance of Fiber Tile Boards Constructions

BMS79

BMS80

BMS81

BMS82

BMS83

BMS84

BMS86

BMS87

Water-Distributing Systems for Buildings

Performance Test of Floor Coverings for Use in Low-Cost Housing: Part 4

Field Inspectors' Check List for Building Constructions (cloth cover, 5 × 73/ inches) --- 30\$

Water Permeability of Walls Built of Masonry Units Strength of Sleeve Joints in Copper Tubing Made With Various Lead-Base Solders... 15\& Survey of Roofing Materials in the South Central States.... Structural, Heat-Transfer, and Water-Permeability Properties of "Speedbrik" Wall Construction Sponsored by the General Shale Products Corporation _.

A Method for Developing Specifications for Building Construction-Report of Subcommittee on Specifications of the Central Housing Committee on Research,

BMS89 Structural Properties of "Precision-Built, Jr." (Second Construction) Prefabricated Wood-Frame Wall Construction Sponsored by the Homasote Co

BMS90 Structural Properties of "PHC" Prefabricated Wood-Frame Construction for Walls, Floors, and Roofs Sponsored by the PHC Housing Corporation......

BMS92 Fire-Resistance Classifications of Building Constructions....

[List continued on cover page Iv] 


\section{BUILDING MATERIALS AND STRUCTURES REPORTS}

[Continued from cover page III]

BMS94 Water Permeability and Weathering Resistance of Stucco-Faced, Gunite-Faced, and "Knap Concrete-Unit" Walls

BMS95

BMS96

Tests of Cement-Water Paints and Other Waterproofings for Unit-Masonry Wails .... Properties of a Porous Concrete of Cement and Uniform-Sized Gravel Structural and Heat-Transfer Properties of "Multiple Box-Girder Plywood Panels" for Walls, Floors, and Roofs

BMS100

BMS101

BMS102

BMS103

BMS104

Relative Slipperiness of Floor and Deck Surfaces

Strength and Resistance to Corrosion of Ties for Cavity Walls

Measurements of Heat Losses From Slab Floors

Structural Properties of Prefabricated Plywood Lightweight Constructions for Walls

Partitions, Floors, and Roofs Sponsored by the Douglas Fir Plywood Association -

BMS105

BMS106

Paint Manual With Particular Reference to Federal Specifications

BMS108

BMS109

BMS110

BMS111

BMS112

BMS113

BMS114

BMS115

BMS116

BMS117

BMS118

BMS119

BMS120

BMS121

BMS122

BMS123

BMS124

BMS125

BMS126

BMS127

BMS128

BMS129

BMS130

BMS131

BMS132

BMS133

Laboratory Observations of Condensation in Wall Specimens

Temperature Distribution in a Test Bungalow With Various Heating Devices

Strength of Houses: Application of Engineering Principles to Structural Design-..-- $\$ 1.50$

Paints for Exterior Masonry Walls

Performance of a Coal-Fired Boiler Converted to Oil

Properties of Some Lightweight-Aggregate Concretes With and Without an Air-Entraining Admixture

Fire Resistance of Structural Clay Tile Partitions

Temperature in a Test Bungalow With Some Radiant and Jacketed Space Heaters.-- 25

A Study of a Baseboard Convector Heating System in a Test Bungalow-1.--

Preparation and Revision of Building Codes

Fire Resistance of Walls of Lightweight Aggregate Conerete Masonry Units

Stack Venting of Plumbing Fixtures

Wet Venting of Plumbing Fixtures

Fire Resistance of Walls of Gravel-Aggregate Concrete Masonry Units

Investigation of Failures of White-Coat Plasters

Physical Properties of Some Samples of Asbestos-Cement Siding.

Fire Tests of Wood-Framed Walls and Partitions With Asbestos-Cement Facings -.-.- 15

Fire Tests of Steel Columns Protected With Siliceous Aggregate Concrete

Stone Exposure Test Wall _.........

The Self-Siphonage of Fixture Traps

Effect of Aging on the Soundness of Regularly Hydrated Dolomitic Lime Putties.-. 15

Atmospheric Exposure Tests of Nailed Sheet Metal Building Materials_..-_-_-

Fire Endurance of Shutters for Moving-Stairway Openings

Methods and Equipment for Testing Printed-Enamel Felt-Base Floor Covering

Fire Tests of Gunite Slabs and Partitions...

Capacities of Plumbing Stacks in Buildings..

Live Loads on Floors in Buildings 